\title{
Catalytic Anti-Markovnikov Transformations of Hindered Terminal Alkenes Enabled by Aldehyde-Selective Wacker-Type Oxidation
}

\author{
Kelly E. Kim, Jiaming Li, Robert H. Grubbs, ${ }^{*}$ and Brian M. Stoltz*
}

The Warren and Katharine Schlinger Laboratory for Chemistry and Chemical Engineering, Division of Chemistry and Chemical Engineering, California Institute of Technology, 1200 E. California Blvd, MC 101-20, Pasadena, CA 91125 (USA)

\section{Table of Contents}

Materials and Methods

Catalyst Optimization

General Experimental Procedures 


\section{Materials and Methods}

Unless noted in the specific procedure, reactions were performed in flame-dried glassware under argon atmosphere. Dried and deoxygenated solvents (Fisher Scientific) were prepared by passage through columns of activated aluminum before use. ${ }^{1}$ Methanol (Fisher Scientific) was distilled from magnesium methoxide immediately prior to use. 1,2-dichloroethane (Fisher Scientific) was distilled from calcium hydride immediately prior to use. Anhydrous ethanol, tert-butanol, and $N, N$-dimethylformamide were purchased from Sigma Aldrich in sure-sealed bottles and used as received unless otherwise noted. Commercial reagents (Sigma Aldrich or Alfa Aesar) were used as received with the exception of palladium(II) acetate (Sigma Aldrich) and XPhos (Sigma Aldrich), which were stored in a nitrogen-filled govebox. The Ohira-Bestmann reagent ${ }^{2}$ and carbomethoxy methylene triphenyl phosphorane $\left(\mathrm{Ph}_{3} \mathrm{P}=\mathrm{CHCO}_{2} \mathrm{Me}\right)^{3}$ were prepared according to known procedures. Triethylamine (Oakwood Chemical) and diisopropylethylamine (Oakwood Chemical) were distilled from calcium hydride immediately prior to use. Brine is defined as a saturated aqueous solution of sodium chloride. Reactions requiring external heat were modulated to the specified temperatures using an IKAmag temperature controller. Reaction progress was monitored by thin-layer chromatography (TLC) or Agilent 1290 UHPLC-LCMS. TLC was performed using E. Merck silica gel 60 F254 precoated plates $(0.25 \mathrm{~mm})$ and visualized by UV fluorescence quenching, potassium permanganate, or $p$-anisaldehyde staining. SiliaFlash P60 Academic Silica gel (particle size 0.040-0.063 mm) was used for flash chromatography. ${ }^{1} \mathrm{H}$ and ${ }^{13} \mathrm{C}$ NMR spectra were recorded on a Varian Inova 500 spectrometer $(500 \mathrm{MHz}$ and $126 \mathrm{MHz}$, respectively), a Bruker AV III HD spectrometer equipped with a Prodigy liquid nitrogen temperature cryoprobe $(400 \mathrm{MHz}$ and $101 \mathrm{MHz}$, respectively), or a Varian Mercury 300 spectrometer $(300 \mathrm{MHz}$ and $75 \mathrm{MHz}$, respectively) and are reported in terms of chemical shift relative to residual $\mathrm{CHCl}_{3}(\delta 7.26$ and $\delta 77.16 \mathrm{ppm}$, respectively). Data for ${ }^{1} \mathrm{H}$ NMR spectra are reported as follows: chemical shift ( $\delta$ ppm) (multiplicity, coupling constant $(\mathrm{Hz})$, integration). Abbreviations are used as follows: $\mathrm{s}=$ singlet, $\mathrm{bs}=$ broad singlet, $\mathrm{d}=$ doublet, $\mathrm{t}=$ triplet, $\mathrm{q}=$ quartet, $\mathrm{m}=$ complex multiplet. Infrared (IR) spectra were recorded on a Perkin Elmer Paragon 1000 spectrometer using thin film samples on $\mathrm{KBr}$ plates, and are reported in frequency of absorption $\left(\mathrm{cm}^{-1}\right)$. High-resolution mass spectra (HRMS) were obtained from the Caltech Mass Spectral Facility using a JEOL JMS-600H High Resolution Mass Spectrometer with fast atom bombardment $(\mathrm{FAB}+)$ ionization mode or were acquired using an Agilent 6200 Series TOF with an Agilent G1978A Multimode source in electrospray ionization (ESI+) mode. 


\section{Catalyst Optimization}

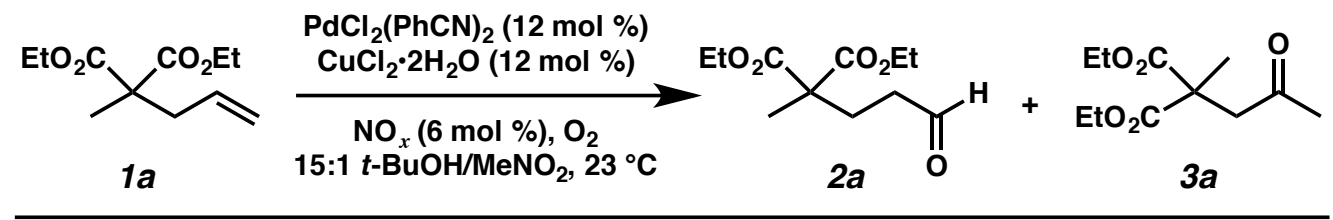

Aldehyde Yield (\%) Oxidation Yield (\%)

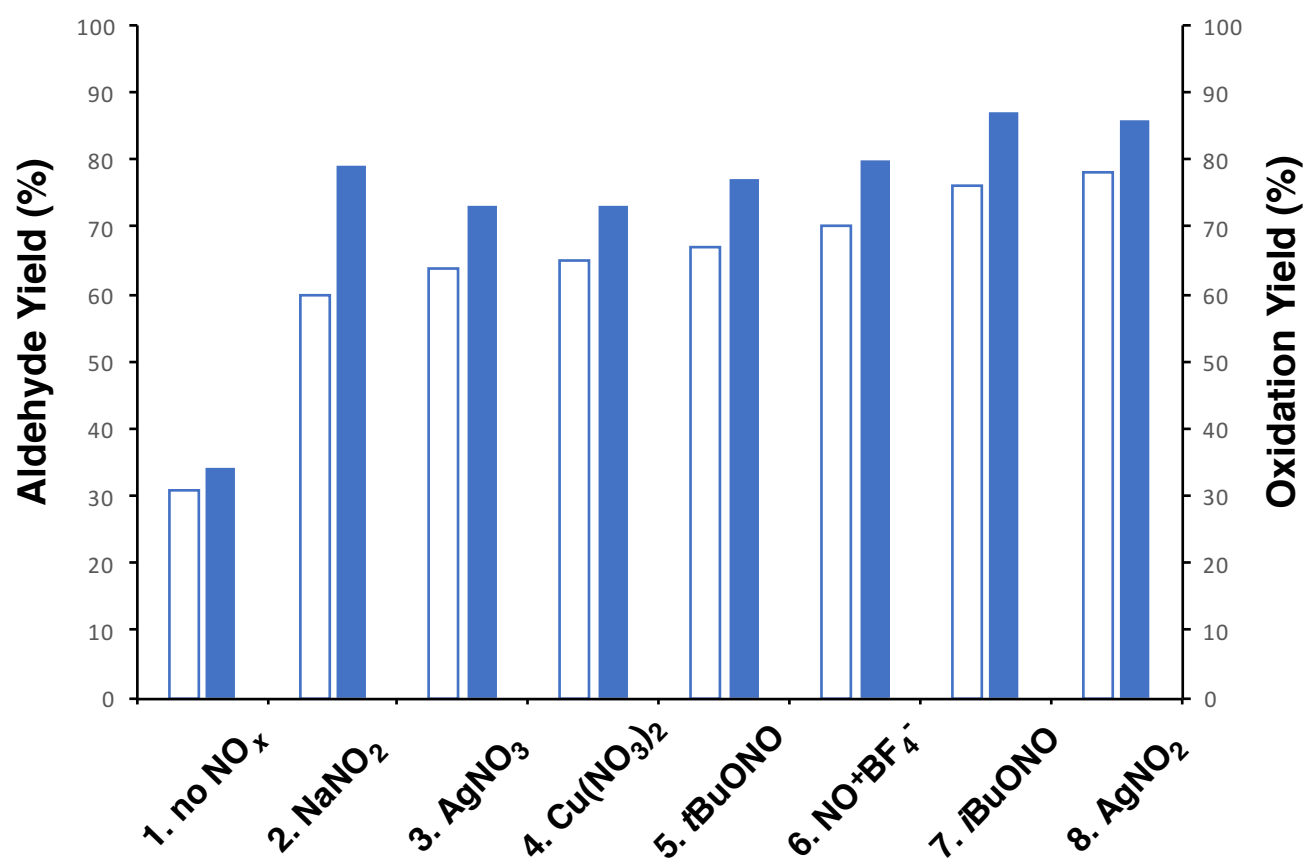

\begin{tabular}{|c|c|c|c|c|}
\hline $\begin{array}{c}\mathrm{NO}_{x} \\
\text { species } \\
\end{array}$ & $\begin{array}{l}\text { Aldehyde } \\
\text { yield }(\%)^{a}\end{array}$ & $\begin{array}{c}\text { Ketone } \\
\text { yield }(\%)\end{array}$ & $\begin{array}{l}\text { Oxidation } \\
\text { yield }(\%)^{b}\end{array}$ & $\begin{array}{c}\text { Selectivity } \\
\text { (aldehyde:ketone) }\end{array}$ \\
\hline $\mathrm{AgNO}_{2}$ & 78 & 8 & 86 & $10: 1$ \\
\hline $\mathrm{AgNO}_{3}$ & 64 & 9 & 73 & $7: 1$ \\
\hline $\mathrm{NaNO}_{2}$ & 60 & 19 & 79 & $3: 1$ \\
\hline $\mathrm{NO}^{+} \mathrm{BF}_{4}^{-}$ & 70 & 10 & 80 & $7: 1$ \\
\hline $\mathrm{Cu}\left(\mathrm{NO}_{3}\right)_{2}$ & 65 & 8 & 73 & $8: 1$ \\
\hline$t \mathrm{BuONO}$ & 67 & 10 & 77 & $7: 1$ \\
\hline$i \mathrm{BuONO}$ & 76 & 11 & 87 & $7: 1$ \\
\hline no $\mathrm{NO}_{x}$ & 31 & 3 & 34 & $10: 1$ \\
\hline
\end{tabular}

${ }^{a}$ Yields were calculated from the crude ${ }^{1} \mathrm{H}$ NMR spectrum.

${ }^{b}$ Oxidation yield is the sum of the yields of aldehyde $\mathbf{2 a}$ and methyl ketone $\mathbf{3 a}$.

Procedure for Catalyst Optimization:

To a flame-dried $25-\mathrm{mL}$ round-bottom flask with a magnetic stir bar were added bis(benzonitrile)palladium(II) chloride $(9.2 \mathrm{mg}, 0.024 \mathrm{mmol}, 0.12$ equiv), copper(II)

chloride dihydrate $(4.1 \mathrm{mg}, 0.024 \mathrm{mmol}, 0.12$ equiv), and silver nitrite (1.8 $\mathrm{mg}, 0.012$ 
mmol, 0.06 equiv). The flask was capped with a rubber septum, and tert-butyl alcohol $(3.75 \mathrm{~mL})$ and nitromethane $(0.25 \mathrm{~mL})$ were added sequentially by syringe. The mixture was stirred at $23{ }^{\circ} \mathrm{C}$ and sparged with oxygen gas (balloon) for 3 minutes. Alkene 1a (42.9 mg, $0.20 \mathrm{mmol}, 1.00$ equiv) was added dropwise by syringe, and the reaction mixture was sparged with oxygen for another minute. The reaction was stirred under oxygen atmosphere at $23{ }^{\circ} \mathrm{C}$ for 14 hours, after which the reaction mixture was diluted with water $(4 \mathrm{~mL})$ and extracted with dichloromethane $(3 \times 5 \mathrm{~mL})$. The organic extracts were dried over sodium sulfate, then filtered and concentrated in vacuo. Nitrobenzene (24.6 mg, $0.20 \mathrm{mmol}, 1.00$ equiv) was added as an internal standard immediately prior to NMR analysis, and the yield and selectivity of the formation of aldehyde $\mathbf{2 a}$ was calculated from the ${ }^{1} \mathrm{H}$ NMR spectrum $(\mathrm{d} 1=15 \mathrm{~s}) .{ }^{4,5}$

\section{General Experimental Procedures}
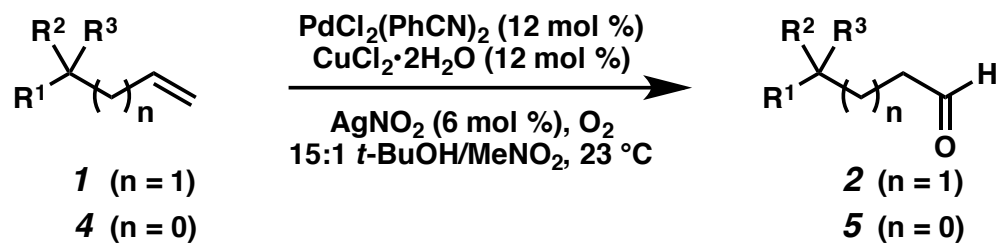

General Procedure A. Aldehyde-selective Wacker-type oxidation of alkenes.

To a flame-dried 25-mL round-bottom flask with a magnetic stir bar were added bis(benzonitrile)palladium(II) chloride $(9.2 \mathrm{mg}, 0.024 \mathrm{mmol}, 0.12 \mathrm{muiv})$, copper(II) chloride dihydrate ( $4.1 \mathrm{mg}, 0.024 \mathrm{mmol}, 0.12$ equiv), and silver nitrite $(1.8 \mathrm{mg}, 0.012$ mmol, 0.06 equiv). The flask was capped with a rubber septum, and tert-butyl alcohol $(3.75 \mathrm{~mL})$ and nitromethane $(0.25 \mathrm{~mL})$ were added sequentially by syringe. The mixture was stirred at $23{ }^{\circ} \mathrm{C}$ and sparged with oxygen gas (balloon) for 3 minutes. Alkene 1 or 4 ( $0.20 \mathrm{mmol}, 1.00$ equiv) was added dropwise by syringe, and the reaction mixture was sparged with oxygen for another minute. The reaction was stirred under oxygen atmosphere at $23{ }^{\circ} \mathrm{C}$ until TLC analysis indicated consumption of starting material. The reaction mixture was diluted with water $(4 \mathrm{~mL})$ and extracted with dichloromethane $(3 \mathrm{x}$ $5 \mathrm{~mL})$. The organic extracts were dried over sodium sulfate, then filtered and concentrated in vacuo. The crude residue was purified by silica gel column chromatography, using mixture of hexanes and ethyl acetate as eluent to afford aldehyde 2 or $5^{6}$

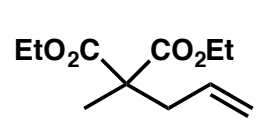

$1 a$

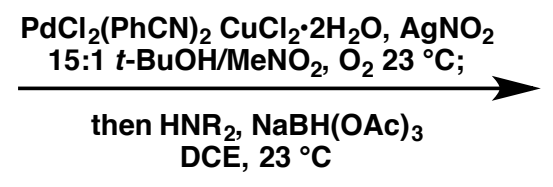

DCE, $23^{\circ} \mathrm{C}$

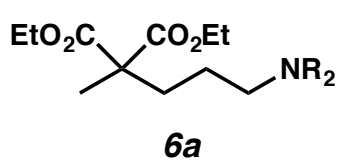

$6 a$

General Procedure B. Hydroamination of diethyl 2-allyl-2-methylmalonate (1a).

To a flame-dried $25-\mathrm{mL}$ round-bottom flask with a magnetic stir bar were added bis(benzonitrile)palladium(II) chloride $(9.2 \mathrm{mg}, 0.024 \mathrm{mmol}, 0.12$ equiv), copper(II) chloride dihydrate ( $4.1 \mathrm{mg}, 0.024 \mathrm{mmol}, 0.12$ equiv), and silver nitrite (1.8 $\mathrm{mg}, 0.012$ mmol, 0.06 equiv). The flask was capped with a rubber septum, and tert-butyl alcohol $(3.75 \mathrm{~mL})$ and nitromethane $(0.25 \mathrm{~mL})$ were added sequentially by syringe. The mixture was stirred at $23{ }^{\circ} \mathrm{C}$ and sparged with oxygen gas (balloon) for 3 minutes. Alkene 1a 
(42.9 mg, $0.20 \mathrm{mmol}, 1.00$ equiv) was added dropwise by syringe, and the reaction mixture was sparged with oxygen for another minute. The reaction was stirred under oxygen atmosphere at $23{ }^{\circ} \mathrm{C}$ for 12 hours, when TLC analysis indicated consumption of starting material. The solvent was removed under reduced pressure, and the residue was loaded onto a short plug of silica gel, eluting with 30\% ethyl acetate in hexanes (100 $\mathrm{mL}$ ). The oil obtained upon concentration was then redissolved in 1,2-dichloroethane (4 $\mathrm{mL})$ and treated with amine $\left(0.22 \mathrm{mmol}, 1.1\right.$ equiv) at $23{ }^{\circ} \mathrm{C}$. After one hour, sodium triacetoxyborohydride $(63.6 \mathrm{mg}, 0.30 \mathrm{mmol}, 1.50$ equiv) was added in one portion. Stirring was continued at $23{ }^{\circ} \mathrm{C}$ for 5 hours, at which time the reaction was diluted with diethyl ether $(3 \mathrm{~mL})$, washed with saturated aqueous sodium bicarbonate $(5 \mathrm{~mL})$, and extracted with diethyl ether $(3 \times 5 \mathrm{~mL})$. The organic extracts were dried over sodium sulfate, then filtered and concentrated under reduced pressure. The crude residue was purified by silica gel column chromatography, using mixture of hexanes and ethyl acetate with $0.5 \%$ triethylamine as eluent to afford amine $\mathbf{6 a}$.

\section{Substrate Synthesis and Characterization Data}

Compounds $1 \mathbf{a}$ and $\mathbf{S 5},{ }^{7} \mathbf{1 e},{ }^{8} \mathbf{1 g},{ }^{9} \mathbf{1 h},{ }^{10} \mathbf{1 i},{ }^{6} \mathbf{1 f},{ }^{9}$ and $\mathbf{4 a - c},{ }^{11} \mathbf{S 6}^{9}$ may be prepared as previously reported by our research group.<smiles>CCOC(=O)C(C)Br</smiles>

S1

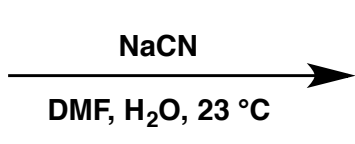

(30\% yield)

\section{Ethyl-2-cyanopropanoate (S2):}

A round-bottom flask equipped with a magnetic stir bar and thermometer was charged with sodium cyanide ( $2.44 \mathrm{~g}, 49.7 \mathrm{mmol}, 1.50$ equiv), $N, N$-dimethylformamide $(22 \mathrm{~mL})$, and water $(2.2 \mathrm{~mL})$. Alkyl bromide $\mathbf{S 1}(4.30 \mathrm{~mL}, 33.1 \mathrm{mmol}, 1.00$ equiv) was added dropwise over 15 minutes, making sure the internal temperature did not exceed $35{ }^{\circ} \mathrm{C}$ throughout addition. After complete addition, the internal thermometer was removed, and the mixture was stirred at $23{ }^{\circ} \mathrm{C}$ for 12 hours, at which time the reaction mixture was diluted with diethyl ether and washed sequentially with cold 5\% aqueous hydrochloric acid $(15 \mathrm{~mL})$ and saturated aqueous sodium bicarbonate $(15 \mathrm{~mL})$. The organic layer was dried over sodium sulfate, then filtered and concentrated under reduced pressure. The crude residue was purified by silica gel column chromatography $(10 \% \rightarrow 20 \%$ ethyl acetate in hexanes), furnishing cyanoester S2 as a colorless oil (1.27 g, 30\% yield). Characterization data match those reported in the literature. ${ }^{12}$ 
<smiles>CCOC(=O)C(C)C#N</smiles>

S2

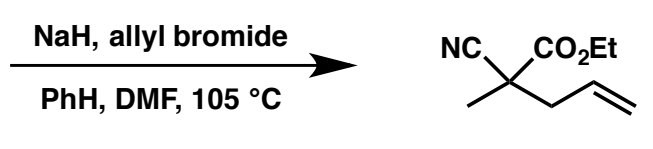

(85\% yield)

Ethyl 2-cyano-2-methylpent-4-enoate (1b):

$1 b$

To a suspension of sodium hydride (60\% dispersion in mineral oil, $419 \mathrm{mg}, 10.5 \mathrm{mmol}$, 1.05 equiv) in benzene $(15 \mathrm{~mL})$ was added a solution of cyanoester $\mathbf{S 2}(1.27 \mathrm{~g}, 9.98$ mmol, 1.00 equiv) in benzene $(12 \mathrm{~mL}) . N, N$-dimethylformamide $(8 \mathrm{~mL})$ was added to stabilize the sodium enolate, and the mixture was stirred at $23{ }^{\circ} \mathrm{C}$ for 20 minutes before allyl bromide ( $910 \mu \mathrm{L} \mathrm{mL}, 10.5 \mathrm{mmol}, 1.05$ equiv) was added dropwise. Upon complete addition, the reaction mixture was heated to reflux $\left(105^{\circ} \mathrm{C}\right)$. After 12 hours, the reaction was allowed to cool to room temperature before quenching with water $(15 \mathrm{~mL})$ and extracting with diethyl ether $(3 \times 20 \mathrm{~mL})$. The organic extracts were washed with brine $(20 \mathrm{~mL})$ and dried over magnesium sulfate before filtration and concentration under reduced pressure. The crude residue was purified by silica gel column chromatography (11\% ethyl acetate in hexanes) to afford alkene $\mathbf{1 b}$ as a colorless oil (1.43 $\mathrm{g}, 85 \%$ yield). $\mathrm{R} f=0.68$ (33\% ethyl acetate in hexanes); ${ }^{1} \mathrm{H} \mathrm{NMR}\left(\mathrm{CDCl}_{3}, 400 \mathrm{MHz}\right) \delta 5.81$ (ddt, $J=$ $16.1,11.0,7.3 \mathrm{~Hz}, 1 \mathrm{H}), 5.33-5.18(\mathrm{~m}, 2 \mathrm{H}), 4.26$ (qd, $J=7.1,1.0 \mathrm{~Hz}, 2 \mathrm{H}), 2.67$ (ddt, $J=$ 13.8, 7.2, $1.2 \mathrm{~Hz}, 1 \mathrm{H}), 2.55-2.45(\mathrm{~m}, 1 \mathrm{H}), 1.58(\mathrm{~s}, 3 \mathrm{H}), 1.32(\mathrm{t}, J=7.1 \mathrm{~Hz}, 3 \mathrm{H}) ;{ }^{13} \mathrm{C}$ NMR $\left(\mathrm{CDCl}_{3}, 101 \mathrm{MHz}\right) \delta 169.0,130.7,121.2,119.8,63.0,43.8,42.2,22.8,14.2$; IR (Neat Film, KBr) 3083, 2985, 1744, 1455, 1233, 1174, 1017, 930; HRMS (FAB+) $m / z$ calc'd for $\mathrm{C}_{9} \mathrm{H}_{14} \mathrm{NO}_{2}[\mathrm{M}+\mathrm{H}]^{+}:$168.1024, found 168.1012 .

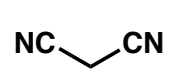

S3

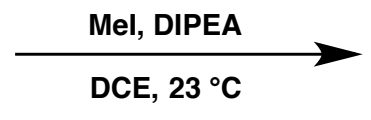

(49\% yield)

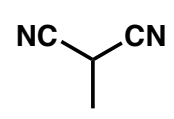

S4

2-Methylmalononitrile (S4):

To a flame-dried round-bottom flask were added malononitrile S3 (3.00 g, 45.4 mmol, 1.00 equiv) and 1,2-dichloroethane $(90 \mathrm{~mL})$. The suspension was cooled to $0{ }^{\circ} \mathrm{C}$ using an ice water bath, and diisopropylethylamine (7.91 mL, $45.4 \mathrm{mmol}, 1.00$ equiv) and methyl iodide (2.83 mL, $45.4 \mathrm{mmol}, 1.00$ equiv) were added dropwise sequentially. The resulting mixture was stirred at $23{ }^{\circ} \mathrm{C}$ for 24 hours, at which time the reaction was quenched with water and transferred to a separatory funnel. The aqueous layer was extracted with ethyl acetate $(5 \times 50 \mathrm{~mL})$, and the combined organic extracts were washed with brine $(50 \mathrm{~mL})$ and dried over sodium sulfate. After filtration and concentration, the crude residue obtained was purified by silica gel column chromatography $(5 \% \rightarrow 10 \% \rightarrow$ $15 \%$ ethyl acetate in hexanes) to furnish 2-methylmalononitrile (S4) as a white solid (1.77 g, 49\% yield). Characterization data match those reported in the literature. ${ }^{13}$ 


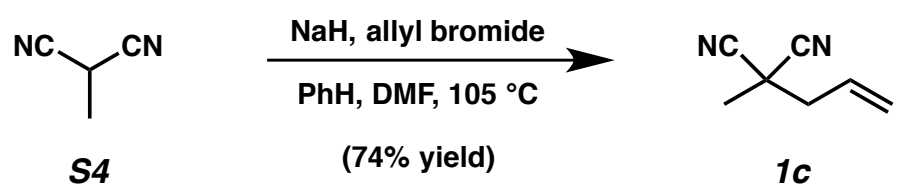

2-Allyl-2-methylmalononitrile (1c):

To a suspension of sodium hydride (60\% dispersion in mineral oil, $309 \mathrm{mg}, 7.72 \mathrm{mmol}$, 1.05 equiv) in benzene $(7.1 \mathrm{~mL})$ was added a solution of 2-methylmalononitrile $\mathbf{S 4}$ (589 $\mathrm{mg}, 7.35 \mathrm{mmol}, 1.00$ equiv) in benzene $(7.1 \mathrm{~mL})$. $N, N$-dimethylformamide $(3.5 \mathrm{~mL})$ was added to stabilize the sodium enolate, and the mixture was stirred at $23{ }^{\circ} \mathrm{C}$ for 20 minutes before allyl bromide ( $670 \mu \mathrm{L} \mathrm{mL}, 7.72 \mathrm{mmol}, 1.05$ equiv) was added dropwise. Upon complete addition, the reaction mixture was heated to reflux $\left(105^{\circ} \mathrm{C}\right)$. After 12 hours, the reaction was allowed to cool to room temperature before quenching with water ( 8 $\mathrm{mL})$ and extracting with diethyl ether $(3 \times 10 \mathrm{~mL})$. The organic extracts were washed with brine $(10 \mathrm{~mL})$ and dried over magnesium sulfate before filtration and concentration under reduced pressure. The crude residue was purified by silica gel column chromatography (10\% ethyl acetate in hexanes) to afford alkene 1c as a colorless oil (653 $\mathrm{mg}, 74 \%$ yield $) . \mathrm{R} f=0.52$ (33\% ethyl acetate in hexanes) ${ }^{1} \mathrm{H} \mathrm{NMR}\left(\mathrm{CDCl}_{3}, 500 \mathrm{MHz}\right) \delta$ 5.89 (ddt, $J=16.7,10.1,7.3 \mathrm{~Hz}, 1 \mathrm{H}), 5.55-5.31$ (m, 2H), 2.68 (ddd, $J=7.3,1.3,0.8 \mathrm{~Hz}$, 2H), $1.79(\mathrm{~s}, 3 \mathrm{H}) ;{ }^{13} \mathrm{C} \mathrm{NMR}\left(\mathrm{CDCl}_{3}, 126 \mathrm{MHz}\right) \delta 128.5,123.6,115.9,43.0,31.7,24.2$; IR (Neat Film, KBr) 3087, 2987, 2927, 1654, 1650, 1454, 1440, 1417, 1276, 1180, 994, 936, 729; HRMS (FAB+) $m / z$ calc'd for $\mathrm{C}_{7} \mathrm{H}_{9} \mathrm{~N}_{2}[\mathrm{M}+\mathrm{H}]^{+}: 121.0760$, found 121.0758.

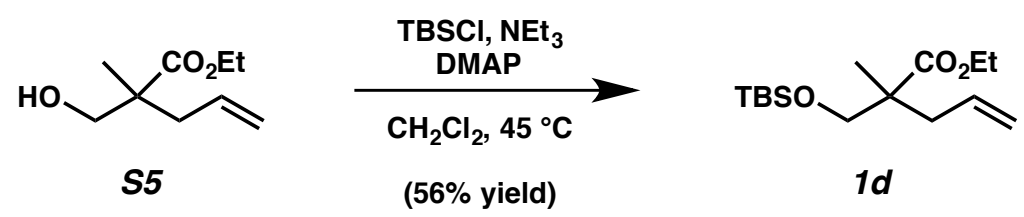

Ethyl 2-(((tert-butyldimethylsilyl)oxy)methyl)-2-methylpent-4-enoate (1d): ${ }^{14}$

To a flame-dried two-necked round-bottom flask equipped with a reflux condenser and magnetic stir bar were added alcohol S5 (108.2 mg, $0.611 \mathrm{mmol}, 1.00$ equiv) and dichloromethane $(12.2 \mathrm{~mL})$. tert-Butyldimethylsilyl chloride $(101.2 \mathrm{mg}, 0.672 \mathrm{mmol}$, 1.10 equiv), triethylamine $(0.17 \mathrm{~mL}, \quad 1.22 \mathrm{mmol}, 2.00$ equiv), and 4(dimethylamino)pyridine $\left(7.5 \mathrm{mg}, 0.0611 \mathrm{mmol}, 0.10\right.$ equiv) were added at $23{ }^{\circ} \mathrm{C}$, and the mixture was heated to reflux $\left(45^{\circ} \mathrm{C}\right)$. After 42 hours, the reaction was allowed to cool to $23{ }^{\circ} \mathrm{C}$ and washed with $2 \mathrm{M}$ aqueous hydrochloric acid $(2 \times 10 \mathrm{~mL})$ and brine $(10$ $\mathrm{mL})$, then dried over sodium sulfate. After filtration and concentration under reduced pressure, the crude residue was purified by silica gel column chromatography (3\% ethyl acetate in hexanes), delivering alkene $\mathbf{1 d}$ as a colorless oil (98.1 mg, 56\% yield). $\mathrm{R} f=$ 0.79 (33\% ethyl acetate in hexanes); ${ }^{1} \mathrm{H}$ NMR $\left(\mathrm{CDCl}_{3}, 400 \mathrm{MHz}\right) \delta 5.72$ (ddt, $J=16.5$, $10.6,7.4 \mathrm{~Hz}, 1 \mathrm{H}), 5.14-4.96(\mathrm{~m}, 2 \mathrm{H}), 4.12(\mathrm{qd}, J=7.2,0.9 \mathrm{~Hz}, 2 \mathrm{H}), 3.70-3.46(\mathrm{~m}, 2 \mathrm{H})$, 2.38 (ddt, $J=13.6,7.2,1.2 \mathrm{~Hz}, 1 \mathrm{H}), 2.22$ (ddt, $J=13.6,7.7,1.1 \mathrm{~Hz}, 1 \mathrm{H}), 1.24$ (t, $J=7.1$ $\mathrm{Hz}, 3 \mathrm{H}), 1.13(\mathrm{~s}, 3 \mathrm{H}), 0.87(\mathrm{~s}, 9 \mathrm{H}), 0.02(\mathrm{~s}, 6 \mathrm{H}) ;{ }^{13} \mathrm{C} \mathrm{NMR}\left(\mathrm{CDCl}_{3}, 101 \mathrm{MHz}\right) \delta 175.8$, 134.1, 118.1, 68.1, 60.4, 48.3, 39.5, 25.9, 19.3, 18.3, 14.4, -5.5; IR (Neat Film, KBr) 2956, 2929, 2857, 1732, 1472, 1386, 1251, 1227, 1101, 837, $776 \mathrm{~cm}^{-1}$; HRMS (ESI+) $m / z$ calc'd for $\mathrm{C}_{15} \mathrm{H}_{31} \mathrm{O}_{3} \mathrm{Si}[\mathrm{M}+\mathrm{H}]^{+}:$287.2037, found 287.2040. 
<smiles>C=CC[C@]1(C)CCc2cc(OC)ccc2C1=O</smiles>

S6

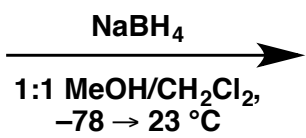

( $86 \%$ yield)<smiles>C=CC[C@]1(C)CCc2cc(OC)ccc2C1O</smiles>

S7

(2S)-2-Allyl-6-methoxy-2-methyl-1,2,3,4-tetrahydronaphthalen-1-ol (S7):

To a solution of ketone $\mathbf{S 6}$ (64.9 $\mathrm{mg}, 0.282 \mathrm{mmol}, 1.00$ equiv) in dichloromethane (2.8 $\mathrm{mL})$ and methanol $(2.8 \mathrm{~mL})$ was added a solution of sodium borohydride $(21.3 \mathrm{mg}, 0.564$ mmol, 2.00 equiv) in dichloromethane $(1.2 \mathrm{~mL})$ and methanol $(1.2 \mathrm{~mL})$ at $-78^{\circ} \mathrm{C}$. The reaction mixture was allowed to warm to $23^{\circ} \mathrm{C}$ over the course of six hours. When TLC analysis indicated full consumption of starting material, the reaction was quenched with acetone $(2.0 \mathrm{~mL})$ and $2 \mathrm{~N} \mathrm{NaOH}(2.0 \mathrm{~mL})$. The phases were separated, and the organic layer was immediately washed with brine $(10 \mathrm{~mL})$ and dried over sodium sulfate. After filtration and concentration under reduced pressure, the crude residue was purified by silica gel column chromatography (15\% ethyl acetate in hexanes), furnishing alcohol S7 as a $1: 1$ mixture of diastereomers $(56.5 \mathrm{mg}, 86 \%$ yield). $\mathrm{R} f=0.26$ (20\% ethyl acetate in hexanes); ${ }^{1} \mathrm{H}$ NMR $\left(\mathrm{CDCl}_{3}, 500 \mathrm{MHz}\right) \delta 7.35(\mathrm{~d}, J=8.5 \mathrm{~Hz}, 1 \mathrm{H}), 7.28$ (d, $J=8.5 \mathrm{~Hz}$, $1 \mathrm{H}), 6.79-6.73(\mathrm{~m}, 2 \mathrm{H}), 6.64(\mathrm{dt}, J=5.1,1.8 \mathrm{~Hz}, 2 \mathrm{H}), 6.04-5.83(\mathrm{~m}, 2 \mathrm{H}), 5.16-5.00(\mathrm{~m}$, $5 \mathrm{H}), 4.23(\mathrm{~s}, 1 \mathrm{H}), 3.78(\mathrm{~s}, 6 \mathrm{H}), 2.87-2.65(\mathrm{~m}, 5 \mathrm{H}), 2.28$ (ddt, $J=13.6,7.3,1.2 \mathrm{~Hz}, 1 \mathrm{H})$, 2.13-2.01 (m, 3H), 1.87 (ddd, $J=13.5,9.4,6.7 \mathrm{~Hz}, 1 \mathrm{H}), 1.78(\mathrm{ddd}, J=13.8,7.5,6.3 \mathrm{~Hz}$, $1 \mathrm{H}$ ), 1.55 (dt, $J=13.4,6.6 \mathrm{~Hz}, 1 \mathrm{H}), 1.46$ (dddd, $J=13.6,5.9,4.7,1.0 \mathrm{~Hz}, 2 \mathrm{H}$ ), 0.99 (s, $3 \mathrm{H}), 0.88(\mathrm{~s}, 4 \mathrm{H}) ;{ }^{13} \mathrm{C} \mathrm{NMR}\left(\mathrm{CDCl}_{3}, 126 \mathrm{MHz}\right) \delta 159.0,158.9,137.7,137.4,135.3$, 135.0, 131.0, 130.9, 130.6, 130.2, 117.7, 117.6, 113.3, 113.2, 112.6, 75.1, 74.9, 55.3, 42.6, 41.6, 37.1, 36.9, 29.4, 29.1, 26.1, 26.0, 21.1, 19.9; IR (Neat Film, KBr) 3430 (br), 2928, 1610, 1501, 1456, 1263, 1159, 1104, 1038, 1015, 912, $802 \mathrm{~cm}^{-1}$; HRMS (FAB+) $m / z$ calc'd for $\mathrm{C}_{15} \mathrm{H}_{20} \mathrm{O}_{2}[\mathrm{M} \bullet]^{+}: 232.1463$, found 232.1439 .<smiles>C=CC[C@]1(C)CCc2cc(OC)ccc2C1O</smiles>

(S)-2-Allyl-6-methoxy-2-methyl-1,2,3,4-tetrahydronaphthalene (1j): $\mathrm{mL})$ was added triethylsilane $(0.12 \mathrm{~mL}, 0.730 \mathrm{mmol}, 3.00$ equiv $)$ and boron trifluoride diethyl etherate $\left(60 \mu \mathrm{L}, 0.486 \mathrm{mmol}, 2.00\right.$ equiv) at $-60^{\circ} \mathrm{C}$. After 10 minutes, the reaction mixture was warmed to $-10^{\circ} \mathrm{C}$ and stirred at this temperature for 7 hours. A saturated aqueous solution of potassium carbonate was added, and the mixture was extracted with dichloromethane $(2 \times 20 \mathrm{~mL})$. The combined organic extracts were dried over sodium sulfate before filtration and concentration under reduced pressure. The crude residue was purified by silica gel column chromatography (5\% ethyl acetate in hexanes), affording tetralin $\mathbf{1 j}$ as a colorless oil $(50.3 \mathrm{mg}$, $96 \%$ yield). $\mathrm{R} f=0.67(20 \%$ ethyl acetate in hexanes); ${ }^{1} \mathrm{H} \mathrm{NMR}\left(\mathrm{CDCl}_{3}, 500 \mathrm{MHz}\right) \delta 6.97(\mathrm{~d}, J=8.3 \mathrm{~Hz}, 1 \mathrm{H}), 6.73-$ $6.63(\mathrm{~m}, 2 \mathrm{H}), 5.91$ (ddt, $J=16.9,10.2,7.5 \mathrm{~Hz}, 1 \mathrm{H}), 5.14-4.96(\mathrm{~m}, 2 \mathrm{H}), 3.79(\mathrm{~s}, 3 \mathrm{H}), 2.79$ (t, $J=6.7 \mathrm{~Hz}, 2 \mathrm{H}), 2.60-2.39$ (m, 2H), 2.06 (qdt, $J=13.7,7.3,1.2 \mathrm{~Hz}, 2 \mathrm{H}), 1.67-1.47$ 
(m, 2H), $0.96(\mathrm{~s}, 3 \mathrm{H}) ;{ }^{13} \mathrm{C} \mathrm{NMR}\left(\mathrm{CDCl}_{3}, 126 \mathrm{MHz}\right) \delta 157.5,137.0,135.2,130.5,128.3$, 117.3, 113.4, 112.0, 55.3, 45.4, 41.1, 33.8, 32.6, 26.5, 24.8; IR (Neat Film, KBr) 3073, 2951, 2914, 1611, 1503, 1464, 1267, 1254, 1236, 1153, 1042, 912, $808 \mathrm{~cm}^{-1}$; HRMS

$(\mathrm{ESI}+) \mathrm{m} / z$ calc' $\mathrm{d}$ for $\mathrm{C}_{15} \mathrm{H}_{21} \mathrm{O}[\mathrm{M}+\mathrm{H}]^{+}: 217.1587$, found $217.1584 ;[\alpha]^{25}{ }_{\mathrm{D}} 6.47(c 1.0$, $\left.\mathrm{CHCl}_{3}\right)$.

\section{Aldehyde Characterization Data}

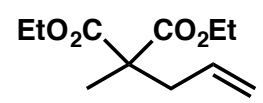

$1 a$

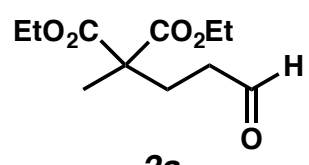

$2 a$

Diethyl 2-methyl-2-(3-oxopropyl)malonate (2a):

Aldehyde 2a was prepared from 1a using General Procedure A, reaction time: $7 \mathrm{~h}$, column eluent: $7 \% \rightarrow 10 \%$ ethyl acetate in hexanes. $90 \%$ isolated yield. $\mathrm{R} f=0.45(33 \%$ ethyl acetate in hexanes); ${ }^{1} \mathrm{H}$ NMR $\left(\mathrm{CDCl}_{3}, 500 \mathrm{MHz}\right) \delta 9.76(\mathrm{t}, J=1.3 \mathrm{~Hz}, 1 \mathrm{H}), 4.18$ (qd, $J=7.2,0.6 \mathrm{~Hz}, 4 \mathrm{H}), 2.56-2.47(\mathrm{~m}, 2 \mathrm{H}), 2.22-2.13(\mathrm{~m}, 2 \mathrm{H}), 1.41(\mathrm{~s}, 3 \mathrm{H}), 1.25(\mathrm{t}, J=$ $7.1 \mathrm{~Hz}, 6 \mathrm{H}) ;{ }^{13} \mathrm{C} \mathrm{NMR}\left(\mathrm{CDCl}_{3}, 126 \mathrm{MHz}\right) \delta 201.1,171.9,61.6,52.9,39.6,27.9,20.5$, 14.2; IR (Neat Film, KBr) 2984, 1730, 1465, 1381, 1262, 1110, 1023, $861 \mathrm{~cm}^{-1}$; HRMS (ESI+) $m / z$ calc'd for $\mathrm{C}_{11} \mathrm{H}_{19} \mathrm{O}_{5}[\mathrm{M}+\mathrm{H}]^{+}: 231.1227$, found 231.1232 .

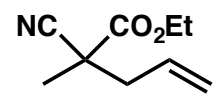

$1 b$

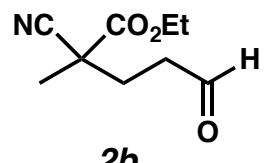

$2 b$

Ethyl 2-cyano-2-methyl-5-oxopentanoate (2b):

Aldehyde 2b was prepared from 1b using General Procedure A, reaction time: $7 \mathrm{~h}$, column eluent: $20 \%$ ethyl acetate in hexanes. $81 \%$ isolated yield. $\mathrm{R} f=0.39(33 \%$ ethyl acetate in hexanes); ${ }^{1} \mathrm{H}$ NMR $\left(\mathrm{CDCl}_{3}, 400 \mathrm{MHz}\right) \delta 9.77(\mathrm{~d}, J=0.9 \mathrm{~Hz}, 1 \mathrm{H}), 4.25$ (qd, $J=$ 7.1, $0.7 \mathrm{~Hz}, 2 \mathrm{H}), 2.83-2.53(\mathrm{~m}, 2 \mathrm{H}), 2.27$ (dddd, $J=14.4,10.0,5.6,0.7 \mathrm{~Hz}, 1 \mathrm{H}), 2.15-$ $2.02(\mathrm{~m}, 1 \mathrm{H}), 1.61(\mathrm{~d}, J=0.7 \mathrm{~Hz}, 3 \mathrm{H}), 1.31(\mathrm{td}, J=7.1,0.7 \mathrm{~Hz}, 3 \mathrm{H}) ;{ }^{13} \mathrm{C} \mathrm{NMR}\left(\mathrm{CDCl}_{3}\right.$, $101 \mathrm{MHz}) \delta 199.2,168.8,119.5,63.2,43.1,39.9,30.1,23.6,14.1$; IR (Neat Film, KBr) 2988, 2944, 1744, 1715, 1453, 1255, 1128, 1017, $857 \mathrm{~cm}^{-1}$; HRMS (FAB+) $\mathrm{m} / z$ calc'd for $\mathrm{C}_{9} \mathrm{H}_{14} \mathrm{NO}_{3}[\mathrm{M}+\mathrm{H}]^{+}:$: 184.0974 , found 184.0976 .

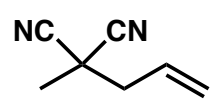

$1 c$

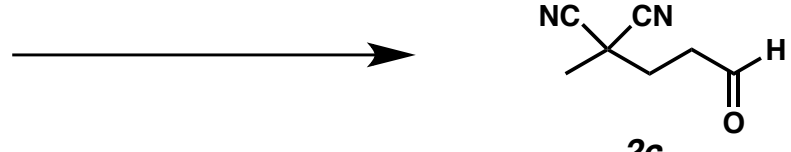

$2 c$

2-Methyl-2-(3-oxopropyl)malononitrile (2c):

Aldehyde 2c was prepared from 1c using General Procedure A, reaction time: $17 \mathrm{~h}$, column eluent: $20 \%$ ethyl acetate in hexanes. $89 \%$ isolated yield. $\mathrm{R} f=0.25$ (33\% ethyl acetate in hexanes); ${ }^{1} \mathrm{H}$ NMR $\left(\mathrm{CDCl}_{3}, 400 \mathrm{MHz}\right) \delta 9.85(\mathrm{~s}, 1 \mathrm{H}), 3.00-2.84(\mathrm{~m}, 2 \mathrm{H})$, 
2.38-2.21 (m, 2H), $1.84(\mathrm{~s}, 3 \mathrm{H}) ;{ }^{13} \mathrm{C} \mathrm{NMR}\left(\mathrm{CDCl}_{3}, 101 \mathrm{MHz}\right) \delta 197.7,115.67,39.9,31.6$, 31.2, 25.0; IR (Neat Film, KBr) 2848, 1724, 1454, 1389, 1150, 897, $629 \mathrm{~cm}^{-1}$; HRMS $(\mathrm{FAB}+) \mathrm{m} / z$ calc'd for $\mathrm{C}_{7} \mathrm{H}_{9} \mathrm{~N}_{2} \mathrm{O}[\mathrm{M}+\mathrm{H}]^{+}:$137.0715, found 137.0688.

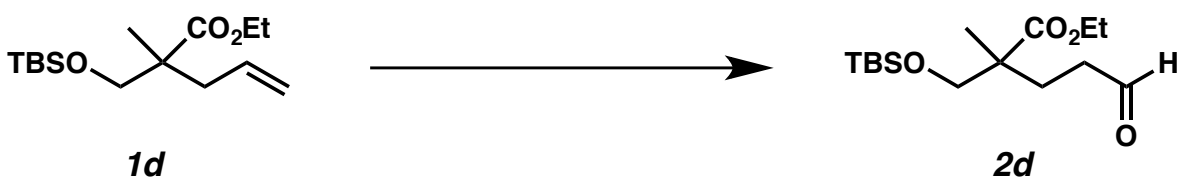

Ethyl 2-(((tert-butyldimethylsilyl)oxy)methyl)-2-methyl-5-oxopentanoate (2d):

Aldehyde 2d was prepared from 1d using General Procedure A, reaction time: $15 \mathrm{~h}$, column eluent: $7 \%$ ethyl acetate in hexanes. $87 \%$ isolated yield. $\mathrm{R} f=0.70(33 \%$ ethyl acetate in hexanes); ${ }^{1} \mathrm{H}$ NMR $\left(\mathrm{CDCl}_{3}, 500 \mathrm{MHz}\right) \delta 9.74(\mathrm{t}, J=1.6 \mathrm{~Hz}, 1 \mathrm{H}), 4.10(\mathrm{q}, J=$ $7.1 \mathrm{~Hz}, 2 \mathrm{H}), 3.64-3.57$ (m, 2H), 2.46-2.40 (m, 2H), 1.97 (ddd, $J=14.0,8.7,7.1 \mathrm{~Hz}, 1 \mathrm{H})$, $1.82-1.72(\mathrm{~m}, 1 \mathrm{H}), 1.23(\mathrm{t}, J=7.1 \mathrm{~Hz}, 3 \mathrm{H}), 1.13(\mathrm{~s}, 3 \mathrm{H}), 0.85(\mathrm{~s}, 9 \mathrm{H}), 0.01 \mathrm{~s}, 6 \mathrm{H}) ;{ }^{13} \mathrm{C}$ NMR $\left(\mathrm{CDCl}_{3}, 126 \mathrm{MHz}\right) \delta 202.1,175.5,68.4,60.7,47.6,39.6,27.2,25.9,19.7,18.3$, 14.3, -5.5; IR (Neat Film, KBr) 2955, 2930, 2857, 1728, 1472, 1252, 1184, 1100, 838, $777,668 \mathrm{~cm}^{-1}$; HRMS (ESI+) $\mathrm{m} / z$ calc'd for $\mathrm{C}_{15} \mathrm{H}_{31} \mathrm{O}_{4} \mathrm{Si}[\mathrm{M}+\mathrm{H}]^{+}: 303.1986$, found 303.1983 .<smiles>C=CC[C@]1(C)CCC(OC(C)(C)C)=CC1=O</smiles>

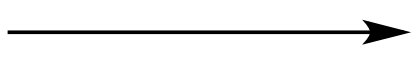

(S)-3-(4-Isobutoxy-1-methyl-2-oxocyclohex-3-en-1-yl)propanal (2e):

Aldehyde 2e was prepared from 1e using General Procedure A, reaction time: $14 \mathrm{~h}$, column eluent: $15 \%$ ethyl acetate in hexanes. $60 \%$ isolated yield. $\mathrm{R} f=0.34(33 \%$ ethyl acetate in hexanes); ${ }^{1} \mathrm{H}$ NMR $\left(\mathrm{CDCl}_{3}, 500 \mathrm{MHz}\right) \delta 9.76(\mathrm{t}, J=1.5 \mathrm{~Hz}, 1 \mathrm{H}), 5.24(\mathrm{~s}, 1 \mathrm{H})$, $3.57(\mathrm{~d}, J=6.5 \mathrm{~Hz}, 2 \mathrm{H}), 2.53-2.36(\mathrm{~m}, 4 \mathrm{H}), 2.02(\mathrm{dq}, J=13.3,6.7 \mathrm{~Hz}, 1 \mathrm{H}), 1.93-1.69$ $(\mathrm{m}, 4 \mathrm{H}), 1.10(\mathrm{~s}, 3 \mathrm{H}), 1.00-0.95(\mathrm{~m}, 6 \mathrm{H}) ;{ }^{13} \mathrm{C} \mathrm{NMR}\left(\mathrm{CDCl}_{3}, 126 \mathrm{MHz}\right) \delta 203.2,202.4$, $176.3,101.5,75.0,42.7,39.4,32.8,29.1,27.9,26.0,22.6,19.2$; IR (Neat Film, KBr) 2961, 2932, 1724, 1648, 1607, 1384, 1369, 1195, 993, $840 \mathrm{~cm}^{-1}$; HRMS (ESI+) $\mathrm{m} / z$ calc'd for $\mathrm{C}_{14} \mathrm{H}_{23} \mathrm{O}_{3}[\mathrm{M}+\mathrm{H}]^{+}:$239.1642, found 239.1638; $[\alpha]^{25}-5.0\left(c 0.94, \mathrm{CHCl}_{3}\right)$.

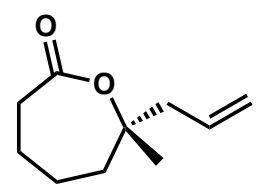

$1 f$

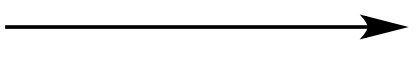

(S)-3-(2-Methyl-7-oxooxepan-2-yl)propanal (2f): column eluent: $20 \% \rightarrow 40 \%$ ethyl acetate in hexanes. $67 \%$ isolated yield. $\mathrm{R} f=0.30$ (67\% ethyl acetate in hexanes); ${ }^{1} \mathrm{H} \mathrm{NMR}\left(\mathrm{CDCl}_{3}, 500 \mathrm{MHz}\right) \delta 9.82(\mathrm{~d}, J=1.2 \mathrm{~Hz}, 1 \mathrm{H})$, 2.80-2.57 (m, 4H), 2.11 (ddd, $J=14.9,9.0,6.3 \mathrm{~Hz}, 1 \mathrm{H}), 1.96-1.74$ (m, 6H), 1.69-1.57 
(m, $1 \mathrm{H}), 1.44(\mathrm{~s}, 3 \mathrm{H}) ;{ }^{13} \mathrm{C} \mathrm{NMR}\left(\mathrm{CDCl}_{3}, 126 \mathrm{MHz}\right) \delta 201.8,174.9,82.3,39.4,38.9,37.6$, 34.9, 24.7, 24.1, 23.7; IR (Neat Film, KBr) 2936, 1720, 1716, 1289, 1185, 1107, 1018, $858 \mathrm{~cm}^{-1}$; HRMS (FAB+) $\mathrm{m} / z$ calc'd for $\mathrm{C}_{10} \mathrm{H}_{17} \mathrm{O}_{3}[\mathrm{M}+\mathrm{H}]^{+}:$: 185.1178, found 185.1177; $[\alpha]^{25} 1.6\left(c 2.46, \mathrm{CHCl}_{3}\right)$.

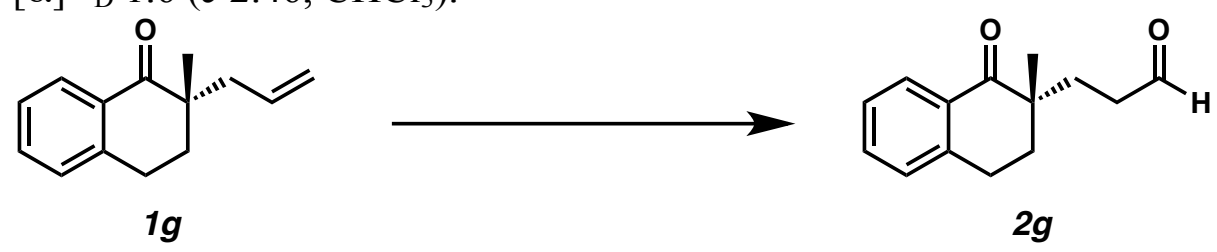

(S)-3-(2-Methyl-1-oxo-1,2,3,4-tetrahydronaphthalen-2-yl)propanal (2g):

Aldehyde $\mathbf{2 g}$ was prepared from $\mathbf{1 g}$ using General Procedure A, reaction time: $12 \mathrm{~h}$, column eluent: $5 \%$ ethyl acetate in hexanes. $80 \%$ isolated yield. $\mathrm{R} f=0.15(20 \%$ ethyl acetate in hexanes); ${ }^{1} \mathrm{H}$ NMR $\left(\mathrm{CDCl}_{3}, 500 \mathrm{MHz}\right) \delta 9.76(\mathrm{t}, J=1.5 \mathrm{~Hz}, 1 \mathrm{H}), 8.01(\mathrm{dd}, J=$ 7.9, $1.4 \mathrm{~Hz}, 1 \mathrm{H}), 7.49-7.42(\mathrm{~m}, 1 \mathrm{H}), 7.33-7.26(\mathrm{~m}, 1 \mathrm{H}), 7.22$ (ddq, $J=7.6,1.5,0.8 \mathrm{~Hz}$, $1 \mathrm{H}), 3.01(\mathrm{t}, J=6.3 \mathrm{~Hz}, 2 \mathrm{H}), 2.61-2.30(\mathrm{~m}, 2 \mathrm{H}), 2.13-1.82(\mathrm{~m}, 4 \mathrm{H}), 1.21(\mathrm{~s}, 3 \mathrm{H}) ;{ }^{13} \mathrm{C}$ NMR $\left(\mathrm{CDCl}_{3}, 126 \mathrm{MHz}\right) \delta 202.2,201.9,143.1,133.4,131.5,128.9,128.1,126.9,44.1$, 39.2, 34.2, 28.8, 25.3, 22.2; IR (Neat Film, KBr) 2929, 1722, 1682, 1600, 1454, 1224, 976, 798, $742 \mathrm{~cm}^{-1}$; HRMS (FAB+) $m / z$ calc'd for $\mathrm{C}_{14} \mathrm{H}_{17} \mathrm{O}_{2}[\mathrm{M}+\mathrm{H}]^{+}: 217.1229$, found $217.1258 ;[\alpha]^{25}-1.0\left(c 1.65, \mathrm{CHCl}_{3}\right)$.

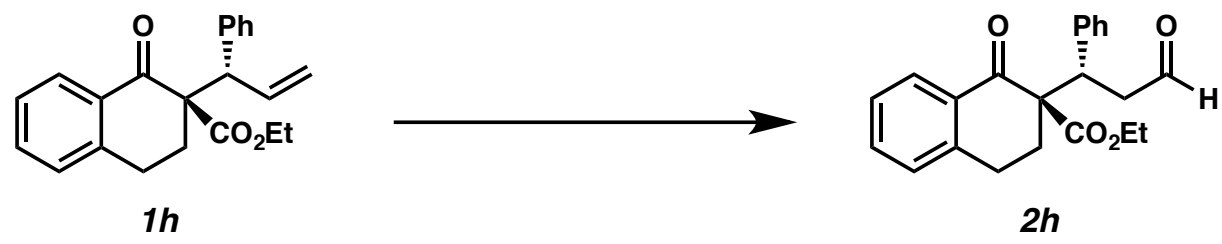

Ethyl (R)-1-oxo-2-((S)-3-oxo-1-phenylpropyl)-1,2,3,4-tetrahydronaphthalene-2carboxylate (2h):

Aldehyde $\mathbf{2 h}$ was prepared from $\mathbf{1 h}$ using General Procedure A, reaction time: $40 \mathrm{~h}$, column eluent: $10 \%$ ethyl acetate in hexanes. $75 \%$ isolated yield. $\mathrm{R} f=0.48(33 \%$ ethyl acetate in hexanes); ${ }^{1} \mathrm{H}$ NMR $\left(\mathrm{CDCl}_{3}, 400 \mathrm{MHz}\right) \delta 9.59(\mathrm{t}, J=1.7 \mathrm{~Hz}, 1 \mathrm{H}), 8.00(\mathrm{dd}, J=$ 7.9, $1.5 \mathrm{~Hz}, 1 \mathrm{H}), 7.44(\mathrm{td}, J=7.5,1.5 \mathrm{~Hz}, 1 \mathrm{H}), 7.40-7.34(\mathrm{~m}, 2 \mathrm{H}), 7.31-7.24(\mathrm{~m}, 2 \mathrm{H})$, 7.24-7.12 (m, 3H), 4.19 (dd, $J=8.4,6.2 \mathrm{~Hz}, 1 \mathrm{H}), 4.08$ (q, $J=7.1 \mathrm{~Hz}, 2 \mathrm{H}), 3.13-3.07$ (m, 2H), 3.07-2.97 (m, 1H), $2.88(\mathrm{dt}, J=17.8,4.5 \mathrm{~Hz}, 1 \mathrm{H}), 2.34$ (ddd, $J=13.7,4.8,3.7 \mathrm{~Hz}$, $1 \mathrm{H}), 1.98$ (ddd, $J=13.8,11.2,5.1 \mathrm{~Hz}, 1 \mathrm{H}), 1.09(\mathrm{t}, J=7.1 \mathrm{~Hz}, 3 \mathrm{H}) ;{ }^{13} \mathrm{C} \mathrm{NMR}\left(\mathrm{CDCl}_{3}\right.$, $101 \mathrm{MHz}) \delta 200.9,194.7,170.2,142.7,139.1,133.6,132.6,130.5,128.7,128.4,128.3$, 127.5, 126.9, 61.8, 60.5, 46.3, 43.0, 30.5, 26.1, 14.0; IR (Neat Film, KBr) 2978, 2725, $1725,1689,1600,1454,1298,1235,1214,1018,909,742,703,648 \mathrm{~cm}^{-1}$; HRMS (ESI+) $m / z$ calc'd for $\mathrm{C}_{22} \mathrm{H}_{23} \mathrm{O}_{5}[\mathrm{M}+\mathrm{OH}]^{+}: 367.1540$, found $367.1535 ;[\alpha]^{25}{ }_{\mathrm{D}} 15.7$ (c 1.52, $\left.\mathrm{CHCl}_{3}\right)$. 


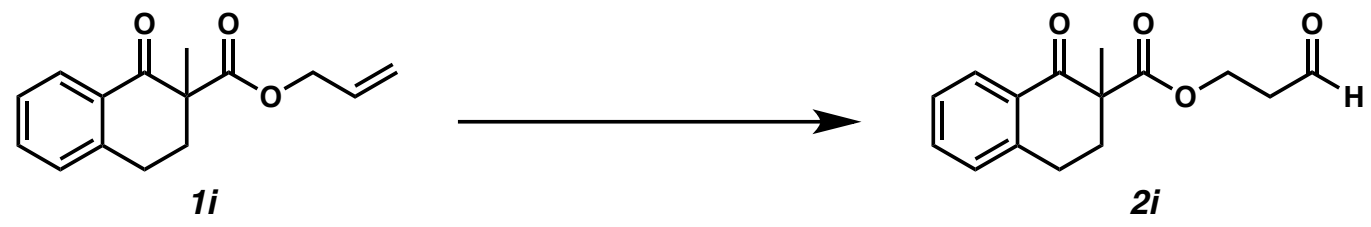

3-Oxopropyl 2-methyl-1-oxo-1,2,3,4-tetrahydronaphthalene-2-carboxylate (2i):

Aldehyde 2i was prepared from 1i using General Procedure A, reaction time: $10 \mathrm{~h}$, column eluent: $15 \%$ ethyl acetate in hexanes. $74 \%$ isolated yield. $\mathrm{R} f=0.27(33 \%$ ethyl acetate in hexanes); ${ }^{1} \mathrm{H} \mathrm{NMR}\left(\mathrm{CDCl}_{3}, 500 \mathrm{MHz}\right) \delta 9.61(\mathrm{t}, J=1.4 \mathrm{~Hz}, 1 \mathrm{H}), 8.02(\mathrm{dd}, J=$ $7.9,1.4 \mathrm{~Hz}, 1 \mathrm{H}), 7.47(\mathrm{td}, J=7.5,1.5 \mathrm{~Hz}, 1 \mathrm{H}), 7.34-7.28(\mathrm{~m}, 1 \mathrm{H}), 7.24-7.19(\mathrm{~m}, 1 \mathrm{H})$, $4.54-4.31(\mathrm{~m}, 2 \mathrm{H}), 3.12-2.86(\mathrm{~m}, 2 \mathrm{H}), 2.68(\mathrm{ddt}, J=7.2,6.0,1.5 \mathrm{~Hz}, 2 \mathrm{H}), 2.58$ (ddd, $J$ $=13.7,6.2,4.9 \mathrm{~Hz}, 1 \mathrm{H}), 2.05(\mathrm{ddt}, J=13.8,9.0,4.6 \mathrm{~Hz}, 1 \mathrm{H}), 1.48(\mathrm{~s}, 3 \mathrm{H}) ;{ }^{13} \mathrm{C} \mathrm{NMR}$ $\left(\mathrm{CDCl}_{3}, 126 \mathrm{MHz}\right) \delta 199.0,196.1,172.9,143.1,133.7,131.6,128.9,128.1,127.0,58.9$, 54.0, 42.5, 33.7, 25.9, 20.4; IR (Neat Film, KBr) 2936, 1732, 1687, 1682, 1601, 1455, $1308,1265,1228,1189,1114,743 \mathrm{~cm}^{-1}$; HRMS $(\mathrm{FAB}+) \mathrm{m} / z$ calc'd for $\mathrm{C}_{15} \mathrm{H}_{17} \mathrm{O}_{4}$ $[\mathrm{M}+\mathrm{H}]^{+}:$261.1127, found 261.1155 .
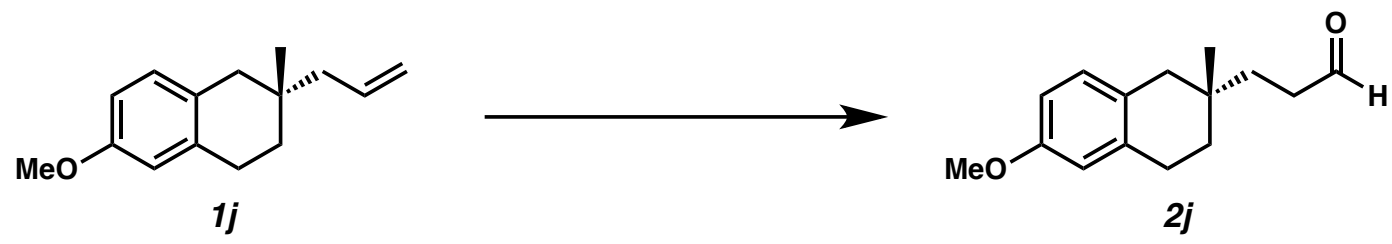

(S)-3-(6-Methoxy-2-methyl-1,2,3,4-tetrahydronaphthalen-2-yl)propanal (2j):

Aldehyde $\mathbf{2} \mathbf{j}$ was prepared from $\mathbf{1 j}$ using General Procedure A, reaction time: $12 \mathrm{~h}$, column eluent: $5 \%$ ethyl acetate in hexanes. $63 \%$ isolated yield. $\mathrm{R} f=0.34(20 \%$ ethyl acetate in hexanes); ${ }^{1} \mathrm{H} \mathrm{NMR}\left(\mathrm{CDCl}_{3}, 500 \mathrm{MHz}\right) \delta 9.79(\mathrm{t}, J=1.9 \mathrm{~Hz}, 1 \mathrm{H}), 6.95(\mathrm{~d}, J=$ $8.4 \mathrm{~Hz}, 1 \mathrm{H}), 6.71-6.66(\mathrm{~m}, 1 \mathrm{H}), 6.64(\mathrm{~d}, J=2.7 \mathrm{~Hz}, 1 \mathrm{H}), 3.77(\mathrm{~s}, 3 \mathrm{H}), 2.77$ (td, $J=6.7$, $4.2 \mathrm{~Hz}, 2 \mathrm{H}), 2.56-2.39(\mathrm{~m}, 4 \mathrm{H}), 1.65-1.60(\mathrm{~m}, 2 \mathrm{H}), 1.58$ (t, $J=6.8 \mathrm{~Hz}, 2 \mathrm{H}), 0.93$ (s, 3H); ${ }^{13} \mathrm{C} \mathrm{NMR}\left(\mathrm{CDCl}_{3}, 126 \mathrm{MHz}\right) \delta 203.0,157.7,136.7,130.5,127.7,113.4,112.2,55.4,41.1$, 39.1, 33.9, 32.6, 31.9, 26.4, 24.4; IR (Neat Film, KBr) 2916, 2834, 2719, 1724, 1610, $1503,1267,1242,1040,808 \mathrm{~cm}^{-1}$; HRMS (FAB+) $\mathrm{m} / z$ calc'd for $\mathrm{C}_{15} \mathrm{H}_{20} \mathrm{O}_{2}[\mathrm{M} \bullet]^{+}$: 232.1463, found 232.1473; $[\alpha]^{25} 85.6\left(c 1.00, \mathrm{CHCl}_{3}\right)$.<smiles>C=CC(C)(C)C(=O)c1ccccc1</smiles>
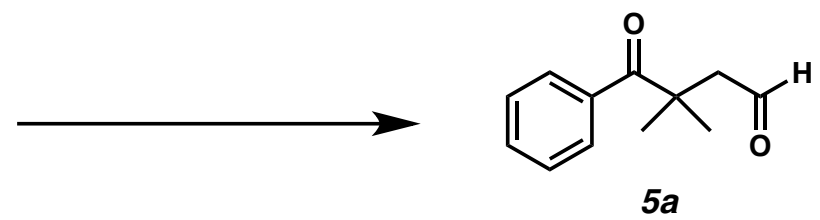

3,3-Dimethyl-4-oxo-4-phenylbutanal (5a):

Aldehyde 5a was prepared from 4a using General Procedure A, reaction time: $20 \mathrm{~h}$, column eluent: $10 \%$ ethyl acetate in hexanes. $85 \%$ isolated yield. $\mathrm{R} f=0.30(33 \%$ ethyl acetate in hexanes); ${ }^{1} \mathrm{H} \mathrm{NMR}\left(\mathrm{CDCl}_{3}, 500 \mathrm{MHz}\right) \delta 9.74(\mathrm{t}, J=1.4 \mathrm{~Hz}, 1 \mathrm{H}), 7.70-7.64$ $(\mathrm{m}, 2 \mathrm{H}), 7.50-7.45(\mathrm{~m}, 1 \mathrm{H}), 7.44-7.38(\mathrm{~m}, 2 \mathrm{H}), 2.83(\mathrm{~d}, J=1.5 \mathrm{~Hz}, 2 \mathrm{H}), 1.46(\mathrm{~s}, 6 \mathrm{H})$; ${ }^{13} \mathrm{C} \mathrm{NMR}\left(\mathrm{CDCl}_{3}, 126 \mathrm{MHz}\right) \delta 208.2,200.6,138.4,131.2,128.3,127.8,54.7,46.1,26.7$; IR (Neat Film, KBr) 2974, 1784, 1712, 1450, 1291, 1114, 967, $714 \mathrm{~cm}^{-1}$; HRMS (ESI+) $m / z$ calc'd for $\mathrm{C}_{12} \mathrm{H}_{15} \mathrm{O}_{2}[\mathrm{M}+\mathrm{H}]^{+}:$191.1067, found 191.1075. 

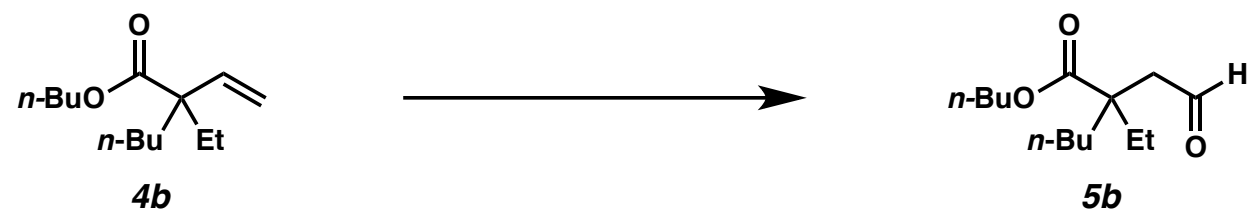

Butyl 2-ethyl-2-(2-oxoethyl)hexanoate (5b):

Aldehyde $\mathbf{5 b}$ was prepared from $\mathbf{4 b}$ using General Procedure A, reaction time: $45 \mathrm{~h}$, column eluent: $10 \%$ ethyl acetate in hexanes. $69 \%$ isolated yield. $\mathrm{R} f=0.36(10 \%$ ethyl acetate in hexanes); ${ }^{1} \mathrm{H} \mathrm{NMR}\left(\mathrm{CDCl}_{3}, 500 \mathrm{MHz}\right) \delta 9.76(\mathrm{t}, J=2.3 \mathrm{~Hz}, 1 \mathrm{H}), 4.10(\mathrm{t}, J=$ $6.6 \mathrm{~Hz}, 2 \mathrm{H}), 2.62(\mathrm{~d}, J=2.3 \mathrm{~Hz}, 2 \mathrm{H}), 1.79-1.56(\mathrm{~m}, 6 \mathrm{H}), 1.42-1.32(\mathrm{~m}, 2 \mathrm{H}), 1.31-1.24$ $(\mathrm{m}, 2 \mathrm{H}), 1.23-1.08(\mathrm{~m}, 2 \mathrm{H}), 0.92(\mathrm{t}, J=7.4 \mathrm{~Hz}, 3 \mathrm{H}), 0.87(\mathrm{t}, J=7.2 \mathrm{~Hz}, 3 \mathrm{H}), 0.83$ (t, $J=$ $7.5 \mathrm{~Hz}, 3 \mathrm{H}) ;{ }^{13} \mathrm{C} \mathrm{NMR}\left(\mathrm{CDCl}_{3}, 126 \mathrm{MHz}\right) \delta 201.7,176.0,64.8,48.1,47.3,35.7,30.7$, 29.0, 26.5, 23.2, 19.3, 14.1, 13.8, 8.7; IR (Neat Film, KBr) 2961, 2936, 2874, 1724, 1459, $1383,1203,1139,1022,737 \mathrm{~cm}^{-1}$; HRMS (ESI+) $m / z$ calc'd for $\mathrm{C}_{14} \mathrm{H}_{26} \mathrm{O}_{3}[\mathrm{M}+\mathrm{H}]^{+}$: 243.1955 , found 243.1961 .<smiles>C=C[C@]1(C)CCc2c3c(cc(OC)c2C1=O)C(C)(C)CCC3</smiles>

$4 c$

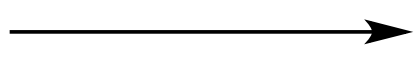

(S)-2-(10-methoxy-2,8,8-trimethyl-1-oxo-1,2,3,4,5,6,7,8-octahydrophenanthren-2yl)acetaldehyde (5c):

Aldehyde 5c was prepared from 4c using General Procedure A, reaction time: $48 \mathrm{~h}$, column eluent: $5 \%$ ethyl acetate in hexanes. $64 \%$ isolated yield. $\mathrm{R} f=0.40(33 \%$ ethyl acetate in hexanes); ${ }^{1} \mathrm{H} \mathrm{NMR}\left(\mathrm{CDCl}_{3}, 500 \mathrm{MHz}\right) \delta 9.89(\mathrm{t}, J=2.5 \mathrm{~Hz}, 1 \mathrm{H}), 6.85(\mathrm{~s}, 1 \mathrm{H})$, $3.88(\mathrm{~s}, 3 \mathrm{H}), 2.80(\mathrm{dd}, J=8.0,4.9 \mathrm{~Hz}, 2 \mathrm{H}), 2.67(\mathrm{dd}, J=15.5,2.3 \mathrm{~Hz}, 1 \mathrm{H}), 2.60-2.45$ $(\mathrm{m}, 3 \mathrm{H}), 2.22-2.11(\mathrm{~m}, 1 \mathrm{H}), 1.96(\mathrm{dt}, J=13.6,4.9 \mathrm{~Hz}, 1 \mathrm{H}), 1.89-1.78(\mathrm{~m}, 2 \mathrm{H}), 1.68-$ $1.61(\mathrm{~m}, 2 \mathrm{H}), 1.30(\mathrm{~s}, 9 \mathrm{H}) ;{ }^{13} \mathrm{C}$ NMR $\left(\mathrm{CDCl}_{3}, 126 \mathrm{MHz}\right) \delta 202.3,200.6,158.9,153.1$, 143.4, 126.3, 118.9, 108.6, 56.0, 51.3, 45.4, 38.4, 35.0, 33.8, 31.8, 31.7, 27.0, 23.7, 22.0, 19.4; IR (Neat Film, KBr) 2959, 2930, 2866, 1717, 1676, 1591, 1558, 1459, 1401, 1318, 1246, 1227, 1104, 1042, 1013, 972, 850, $734 \mathrm{~cm}^{-1}$; HRMS (ESI+) $\mathrm{m} / z$ calc'd for $\mathrm{C}_{20} \mathrm{H}_{26} \mathrm{O}_{3}[\mathrm{M}+\mathrm{H}]^{+}:$315.1955, found 315.1947; $[\alpha]^{25} 4.03\left(c 1.00, \mathrm{CHCl}_{3}\right)$. 
Amine Characterization Data

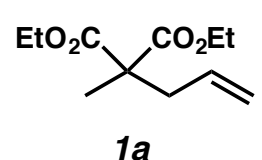

1 a

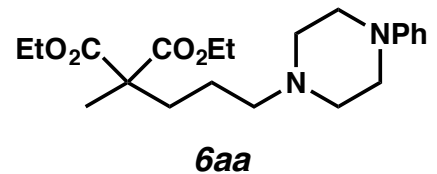

$6 a a$

Diethyl 2-methyl-2-(3-(4-phenylpiperazin-1-yl)propyl)malonate (6aa):

Amine 6aa was prepared from 1a using General Procedure B, column eluent: $25 \%$ ethyl acetate in hexanes with $0.5 \%$ triethylamine. $98 \%$ isolated yield. $\mathrm{R}_{f}=0.16$ (33\% ethyl acetate in hexanes); ${ }^{1} \mathrm{H}$ NMR $\left(\mathrm{CDCl}_{3}, 400 \mathrm{MHz}\right) \delta 7.29-7.18(\mathrm{~m}, 2 \mathrm{H}), 6.92(\mathrm{dt}, J=7.9$, $1.0 \mathrm{~Hz}, 2 \mathrm{H}), 6.88-6.79(\mathrm{~m}, 1 \mathrm{H}), 4.18(\mathrm{q}, J=7.1 \mathrm{~Hz}, 4 \mathrm{H}), 3.26-3.12$ (m, 4H), 2.66-2.53 (m, 4H), 2.45-2.33 (m, 2H), 1.94-1.82 (m, 2H), 1.55-1.44 (m, 2H), $1.41(\mathrm{~d}, J=4.4 \mathrm{~Hz}$, $3 \mathrm{H}), 1.25(\mathrm{t}, J=7.1 \mathrm{~Hz}, 6 \mathrm{H}) ;{ }^{13} \mathrm{C} \mathrm{NMR}\left(\mathrm{CDCl}_{3}, 101 \mathrm{MHz}\right) \delta 172.4,151.4,129.2,119.8$, 116.1, 61.3, 58.7, 53.6, 53.3, 49.2, 33.5, 21.9, 20.1, 14.2; IR (Neat Film, KBr) 2816, 1731, $1600,1502,1257,1235,1110,759,692 \mathrm{~cm}^{-1}$; HRMS (ESI +$) \mathrm{m} / z$ calc'd for $\mathrm{C}_{21} \mathrm{H}_{33} \mathrm{~N}_{2} \mathrm{O}_{5}$ $[\mathrm{M}+\mathrm{OH}]^{+}:$393.2384, found 393.2386.

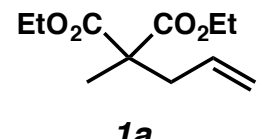

$1 a$

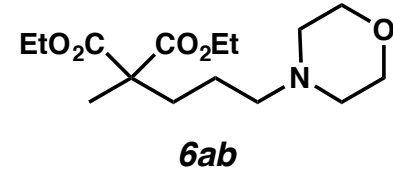

$6 a b$

Diethyl 2-methyl-2-(3-morpholinopropyl)malonate (6ab):

Amine 6ab was prepared from 1a using General Procedure B, column eluent: $8 \% \rightarrow 25 \%$ ethyl acetate in hexanes with $0.5 \%$ triethylamine. $91 \%$ isolated yield. ${ }^{1} \mathrm{H} \mathrm{NMR}\left(\mathrm{CDCl}_{3}\right.$, $300 \mathrm{MHz}) \delta 4.17(\mathrm{q}, J=7.1 \mathrm{~Hz}, 4 \mathrm{H}), 3.76-3.65(\mathrm{~m}, 4 \mathrm{H}), 2.41(\mathrm{dd}, J=5.8,3.6 \mathrm{~Hz}, 4 \mathrm{H})$, $2.37-2.29(\mathrm{~m}, 2 \mathrm{H}), 1.89-1.81(\mathrm{~m}, 2 \mathrm{H}), 1.52-1.36(\mathrm{~m}, 5 \mathrm{H}), 1.23(\mathrm{t}, J=7.1 \mathrm{~Hz}, 6 \mathrm{H})$; ${ }^{13} \mathrm{C} \mathrm{NMR}\left(\mathrm{CDCl}_{3}, 101 \mathrm{MHz}\right) \delta 172.4,66.8,61.4,58.9,53.6,53.5,33.4,21.4,20.1,14.2$; IR (Neat Film, KBr) 2958, 1730, 1457, 1256, 1232, 1118, 1023, $862 \mathrm{~cm}^{-1}$; HRMS (ESI+) $m / z$ calc'd for $\mathrm{C}_{15} \mathrm{H}_{28} \mathrm{NO}_{5}[\mathrm{M}+\mathrm{H}]^{+}: 302.1962$, found 302.1961 .

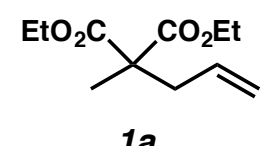

$1 a$

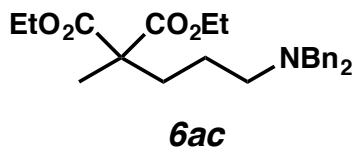

6ac

Diethyl 2-(3-(dibenzylamino)propyl)-2-methylmalonate (6ac):

Amine 6ac was prepared from 1a using General Procedure B, column eluent: 8\% ethyl acetate in hexanes with $0.5 \%$ triethylamine. $76 \%$ isolated yield. $\mathrm{R} f=0.72(33 \%$ ethyl acetate in hexanes); ${ }^{1} \mathrm{H}$ NMR $\left(\mathrm{CDCl}_{3}, 500 \mathrm{MHz}\right) \delta 7.38-7.27(\mathrm{~m}, 8 \mathrm{H}), 7.25-7.20(\mathrm{~m}$, $2 \mathrm{H}), 4.22-4.10(\mathrm{~m}, 4 \mathrm{H}), 3.54(\mathrm{~s}, 4 \mathrm{H}), 2.43(\mathrm{t}, J=7.0 \mathrm{~Hz}, 2 \mathrm{H}), 1.88-1.80(\mathrm{~m}, 2 \mathrm{H}), 1.50-$ $1.41(\mathrm{~m}, 2 \mathrm{H}), 1.38(\mathrm{~d}, J=0.8 \mathrm{~Hz}, 3 \mathrm{H}), 1.22(\mathrm{td}, J=7.1,0.6 \mathrm{~Hz}, 6 \mathrm{H}) ;{ }^{13} \mathrm{C} \mathrm{NMR}\left(\mathrm{CDCl}_{3}\right.$, $126 \mathrm{MHz}) \delta 172.5,139.8,128.9,128.3,126.9,61.2,58.3,53.6,53.5,33.3,21.9,20.1$, 14.2; IR (Neat Film, KBr) 2981, 2796, 1731, 1453, 1245, 1111, 1028, 746, $699 \mathrm{~cm}^{-1}$; HRMS (ESI+) $m / z$ calc'd for $\mathrm{C}_{25} \mathrm{H}_{34} \mathrm{NO}_{4}[\mathrm{M}+\mathrm{H}]^{+}: 412.2482$, found 412.2494 . 

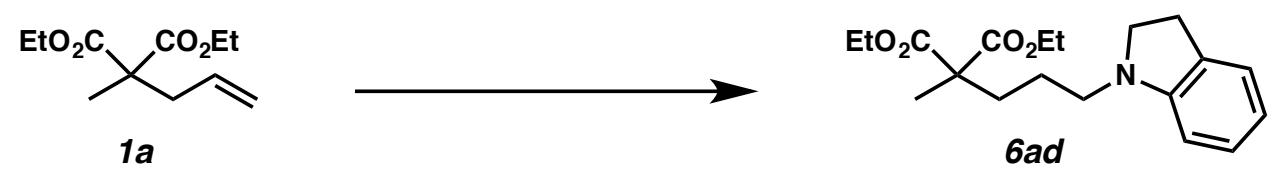

Diethyl 2-(3-(indolin-1-yl)propyl)-2-methylmalonate (6ad):

Amine 6ad was prepared from 1a using General Procedure B, column eluent: 6\% ethyl acetate in hexanes with $0.5 \%$ triethylamine. $96 \%$ isolated yield. $\mathrm{R}_{f}=0.66$ (33\% ethyl acetate in hexanes); ${ }^{1} \mathrm{H}$ NMR $\left(\mathrm{CDCl}_{3}, 400 \mathrm{MHz}\right) \delta 7.12-7.00(\mathrm{~m}, 2 \mathrm{H}), 6.64(\mathrm{t}, J=7.5$ $\mathrm{Hz}, 1 \mathrm{H}), 6.45$ (d, $J=7.8 \mathrm{~Hz}, 1 \mathrm{H}), 4.18$ (q, $J=7.1 \mathrm{~Hz}, 4 \mathrm{H}), 3.32$ (t, $J=8.3 \mathrm{~Hz}, 2 \mathrm{H}), 3.06$ (t, $J=7.2 \mathrm{~Hz}, 2 \mathrm{H}), 2.95(\mathrm{t}, J=8.2 \mathrm{~Hz}, 2 \mathrm{H}), 2.01-1.89(\mathrm{~m}, 2 \mathrm{H}), 1.61-1.54(\mathrm{~m}, 2 \mathrm{H}), 1.43$ $(\mathrm{s}, 3 \mathrm{H}), 1.25(\mathrm{t}, J=7.1 \mathrm{~Hz}, 7 \mathrm{H}) ;{ }^{13} \mathrm{C}$ NMR $\left(\mathrm{CDCl}_{3}, 101 \mathrm{MHz}\right) \delta 172.5,152.7,130.2$, 127.4, 124.5, 117.5, 107.0, 61.4, 53.6, 53.1, 49.6, 33.3, 28.7, 22.5, 20.2, 14.2; IR (Neat Film, KBr) 2980, 1730, 1607, 1490, 1254, 1232, 1113, 1022, $746 \mathrm{~cm}^{-1}$; HRMS (ESI+) $m / z$ calc'd for $\mathrm{C}_{19} \mathrm{H}_{28} \mathrm{NO}_{4}[\mathrm{M}+\mathrm{H}]^{+}:$334.2013, found 334.2019.
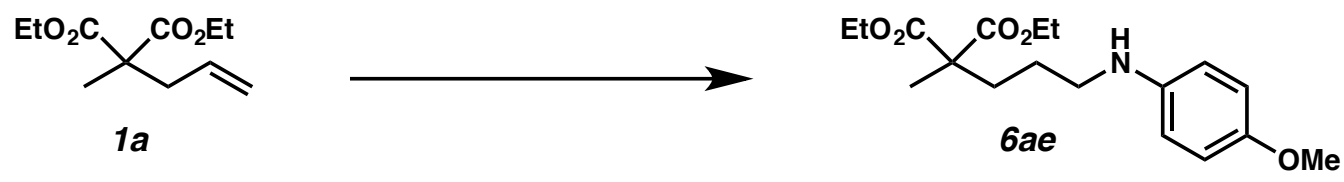

Diethyl 2-(3-((4-methoxyphenyl)amino)propyl)-2-methylmalonate (6ae):

Amine 6ae was prepared from 1a using General Procedure B, column eluent: $10 \%$ ethyl acetate in hexanes with $0.5 \%$ triethylamine. $86 \%$ isolated yield. $\mathrm{R}_{f}=0.45(33 \%$ ethyl acetate in hexanes); ${ }^{1} \mathrm{H}$ NMR $\left(\mathrm{CDCl}_{3}, 400 \mathrm{MHz}\right) \delta 6.81-6.71(\mathrm{~m}, 2 \mathrm{H}), 6.60-6.51(\mathrm{~m}$, $2 \mathrm{H}), 4.17(\mathrm{q}, J=7.1 \mathrm{~Hz}, 4 \mathrm{H}), 3.74(\mathrm{~s}, 3 \mathrm{H}), 3.08(\mathrm{t}, J=6.9 \mathrm{~Hz}, 2 \mathrm{H}), 2.00-1.89(\mathrm{~m}, 2 \mathrm{H})$, $1.62-1.48(\mathrm{~m}, 2 \mathrm{H}), 1.41(\mathrm{~s}, 3 \mathrm{H}), 1.23(\mathrm{t}, J=7.1 \mathrm{~Hz}, 7 \mathrm{H}) ;{ }^{13} \mathrm{C} \mathrm{NMR}\left(\mathrm{CDCl}_{3}, 101 \mathrm{MHz}\right) \delta$ 172.4, 152.2, 142.6, 115.1, 114.2, 61.4, 56.0, 53.6, 45.1, 33.3, 24.7, 20.1, 14.2; IR (Neat Film, KBr) 2982, 1730, 1514, 1235, 1187, 1110, 1037, $820 \mathrm{~cm}^{-1}$; HRMS (ESI+) $\mathrm{m} / \mathrm{z}$ calc'd for $\mathrm{C}_{18} \mathrm{H}_{28} \mathrm{NO}_{5}[\mathrm{M}+\mathrm{H}]^{+}: 338.1962$, found 338.1953 .
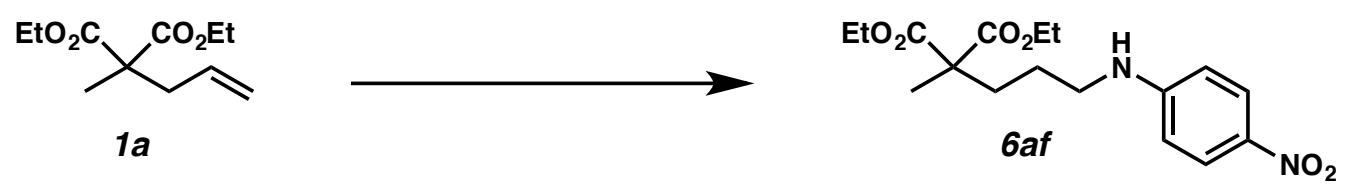

Diethyl 2-methyl-2-(3-((4-nitrophenyl)amino)propyl)malonate (6af):

Amine 6af was prepared from 1a using General Procedure B, column eluent: $10 \% \rightarrow$ $20 \%$ ethyl acetate in hexanes with $0.5 \%$ triethylamine. $95 \%$ isolated yield. $\mathrm{R} f=0.31$ (33\% ethyl acetate in hexanes); ${ }^{1} \mathrm{H}$ NMR $\left(\mathrm{CDCl}_{3}, 500 \mathrm{MHz}\right) \delta 8.12-8.04(\mathrm{~m}, 2 \mathrm{H}), 6.54$ $6.48(\mathrm{~m}, 2 \mathrm{H}), 4.18(\mathrm{q}, J=7.1 \mathrm{~Hz}, 4 \mathrm{H}), 3.22(\mathrm{t}, J=6.8 \mathrm{~Hz}, 2 \mathrm{H}), 1.98-1.92(\mathrm{~m}, 2 \mathrm{H}), 1.69-$ $1.61(\mathrm{~m}, 2 \mathrm{H}), 1.43(\mathrm{~s}, 3 \mathrm{H}), 1.24(\mathrm{t}, J=7.1 \mathrm{~Hz}, 6 \mathrm{H}) ;{ }^{13} \mathrm{C} \mathrm{NMR}\left(\mathrm{CDCl}_{3}, 126 \mathrm{MHz}\right) \delta 172.3$, 153.3, 138.1, 126.6, 111.1, 61.6, 53.5, 43.5, 33.1, 24.2, 20.2, 14.2; IR (Neat Film, KBr) $3383,2836,1748,1721,1610,1475,1314,1328,1190,1114,829 \mathrm{~cm}^{-1}$; HRMS (ESI + ) $m / z$ calc'd for $\mathrm{C}_{17} \mathrm{H}_{25} \mathrm{~N}_{2} \mathrm{O}_{6}[\mathrm{M}+\mathrm{H}]^{+}: 353.1707$, found 353.1707. 
Alkene Transformation Procedures and Characterization Data

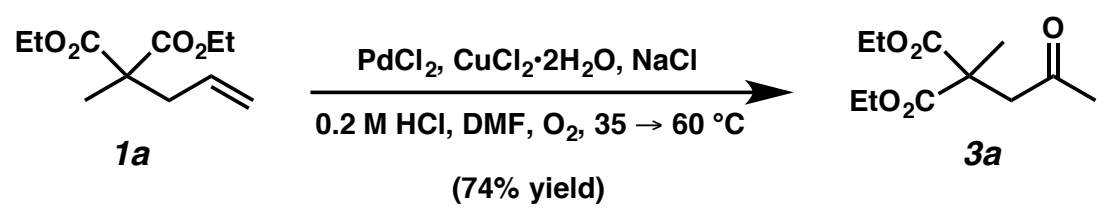

Diethyl 2-methyl-2-(2-oxopropyl)malonate (3a):

To a two-necked round-bottom flask were added palladium(II) chloride (10.6 mg, 0.06 mmol, 0.30 equiv), copper(II) chloride dihydrate (20.5 mg, $0.12 \mathrm{mmol}, 0.60$ equiv), and sodium chloride $(15.0 \mathrm{mg}, 0.26 \mathrm{mmol}, 1.30$ equiv). The mixture was diluted with $0.2 \mathrm{M}$ aqueous hydrochloric acid $(3.1 \mathrm{~mL})$ and stirred vigorously at $35{ }^{\circ} \mathrm{C}$ under oxygen atmosphere (balloon) for 30 minutes. Alkene $1 \mathrm{a}(42.9 \mathrm{mg}, 0.20 \mathrm{mmol}, 1.00$ equiv) was added as a solution in $N, N$-dimethylformamide $(1.0 \mathrm{~mL})$, and the resulting solution was heated stirred vigorously under oxygen atmosphere at $60{ }^{\circ} \mathrm{C}$ for 6 hours. The reaction mixture was allowed to cool to $23{ }^{\circ} \mathrm{C}$ and extracted with chloroform $(2 \times 5 \mathrm{~mL})$. The organic extracts were dried over magnesium sulfate, filtered, and concentrated. The crude residue was purified by silica gel column chromatography ( $8 \%$ ethyl acetate in hexanes) to afford ketone 3a as a colorless oil (34.3 mg, 74\% yield). $\mathrm{R} f=0.24$ (33\% ethyl acetate in hexanes); ${ }^{1} \mathrm{H}$ NMR $\left(\mathrm{CDCl}_{3}, 500 \mathrm{MHz}\right) \delta 4.18(\mathrm{q}, J=7.1 \mathrm{~Hz}, 4 \mathrm{H}), 3.08(\mathrm{~s}$, $2 \mathrm{H}), 2.15(\mathrm{~s}, 3 \mathrm{H}), 1.51(\mathrm{~s}, 3 \mathrm{H}), 1.24(\mathrm{t}, J=7.1 \mathrm{~Hz}, 6 \mathrm{H}) ;{ }^{13} \mathrm{C} \mathrm{NMR}\left(\mathrm{CDCl}_{3}, 126 \mathrm{MHz}\right) \delta$ 205.1, 171.6, 61.7, 51.6, 48.8, 30.5, 20.6, 14.1; IR (Neat Film, KBr) 2984, 1732, 1463 , 1376, 1242, 1109, 1024, 863, $798 \mathrm{~cm}^{-1}$; HRMS (ESI+) $\mathrm{m} / z$ calc'd for $\mathrm{C}_{11} \mathrm{H}_{19} \mathrm{O}_{5}[\mathrm{M}+\mathrm{H}]^{+}$: 231.1227, found 231.1226.

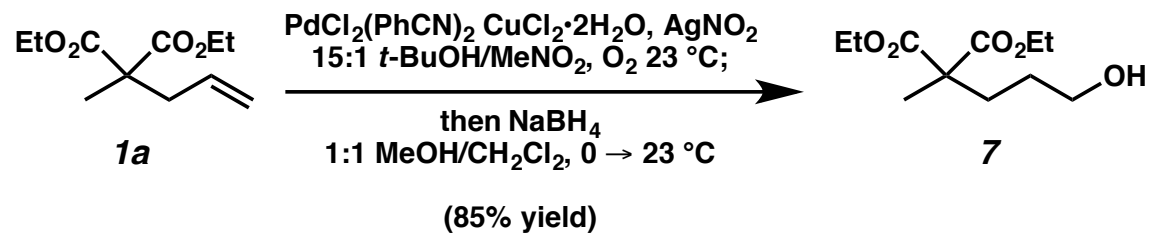

Diethyl 2-(3-hydroxypropyl)-2-methylmalonate (7):

To a flame-dried 25-mL round-bottom flask with a magnetic stir bar were added bis(benzonitrile)palladium(II) chloride (9.2 mg, $0.024 \mathrm{mmol}, 0.12$ equiv), copper(II) chloride dihydrate ( $4.1 \mathrm{mg}, 0.024 \mathrm{mmol}, 0.12$ equiv), and silver nitrite (1.8 $\mathrm{mg}, 0.012$ mmol, 0.06 equiv). The flask was capped with a rubber septum, and tert-butyl alcohol $(3.75 \mathrm{~mL})$ and nitromethane $(0.25 \mathrm{~mL})$ were added sequentially by syringe. The mixture was stirred at $23{ }^{\circ} \mathrm{C}$ and sparged with oxygen gas (balloon) for 3 minutes. Alkene $\mathbf{1 a}$ (42.9 mg, $0.20 \mathrm{mmol}, 1.00$ equiv) was added dropwise by syringe, and the reaction mixture was sparged with oxygen for another minute. The reaction was stirred under oxygen atmosphere at $23{ }^{\circ} \mathrm{C}$ for 12 hours, when TLC analysis indicated consumption of starting material. The solvent was removed under reduced pressure, and the residue was loaded onto a short plug of silica gel, eluting with $30 \%$ ethyl acetate in hexanes (100 $\mathrm{mL})$. The oil obtained upon concentration was then redissolved in $1: 1 \mathrm{MeOH} / \mathrm{CH}_{2} \mathrm{Cl}_{2}(4$ $\mathrm{mL}$ total volume) and cooled to $0{ }^{\circ} \mathrm{C}$ using an ice water bath. Sodium borohydride (11.3 $\mathrm{mg}, 0.30 \mathrm{mmol}, 1.50$ equiv) was added in one portion, and the resulting mixture was 
stirred at $23{ }^{\circ} \mathrm{C}$ for 2 hours, at which time the reaction was quenched with acetone and 2 $\mathrm{N}$ aqueous sodium hydroxide $(2 \mathrm{~mL})$. The phases were separated, and the organic layer was immediately washed with brine $(5 \mathrm{~mL})$ and dried over sodium sulfate. Filtration and concentration delivered the crude product, which was purified by silica gel column chromatography (35\% ethyl acetate in hexanes) to afford alcohol 7 as a colorless oil (39.7 mg, $85 \%$ yield). $\mathrm{R} f=0.18$ (33\% ethyl acetate in hexanes); ${ }^{1} \mathrm{H} \mathrm{NMR}\left(\mathrm{CDCl}_{3}, 300 \mathrm{MHz}\right) \delta$ 4.18 (q, $J=7.1 \mathrm{~Hz}, 4 \mathrm{H}), 3.64(\mathrm{t}, J=6.4 \mathrm{~Hz}, 2 \mathrm{H}), 1.98-1.88(\mathrm{~m}, 2 \mathrm{H}), 1.59-1.49(\mathrm{~m}, 2 \mathrm{H})$, $1.42(\mathrm{~d}, J=2.4 \mathrm{~Hz}, 3 \mathrm{H}), 1.24(\mathrm{t}, J=7.1 \mathrm{~Hz}, 6 \mathrm{H}) ;{ }^{13} \mathrm{C} \mathrm{NMR}\left(\mathrm{CDCl}_{3}, 75 \mathrm{MHz}\right) \delta 172.5$, 62.9, 61.4, 53.5, 32.0, 27.8, 20.1, 14.2; IR (Neat Film, KBr) 3469 (br), 2982, 2939, 1730, 1460, 1270, 1119, 1020, $859 \mathrm{~cm}^{-1}$; HRMS (FAB+) $\mathrm{m} / z$ calc'd for $\mathrm{C}_{11} \mathrm{H}_{21} \mathrm{O}_{5}[\mathrm{M}+\mathrm{H}]^{+}$: 233.1389, found 233.1382 .

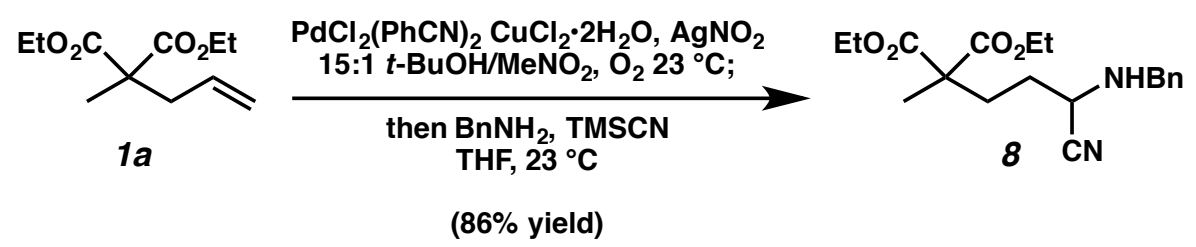

Diethyl 2-(3-(benzylamino)-3-cyanopropyl)-2-methylmalonate (8):

To a flame-dried $25-\mathrm{mL}$ round-bottom flask with a magnetic stir bar were added bis(benzonitrile)palladium(II) chloride $(9.2 \mathrm{mg}, 0.024 \mathrm{mmol}, 0.12$ equiv), copper(II) chloride dihydrate $(4.1 \mathrm{mg}, 0.024 \mathrm{mmol}, 0.12$ equiv), and silver nitrite $(1.8 \mathrm{mg}, 0.012$ mmol, 0.06 equiv). The flask was capped with a rubber septum, and tert-butyl alcohol $(3.75 \mathrm{~mL})$ and nitromethane $(0.25 \mathrm{~mL})$ were added sequentially by syringe. The mixture was stirred at $23{ }^{\circ} \mathrm{C}$ and sparged with oxygen gas (balloon) for 3 minutes. Alkene 1a (42.9 mg, $0.20 \mathrm{mmol}, 1.00$ equiv) was added dropwise by syringe, and the reaction mixture was sparged with oxygen for another minute. The reaction was stirred under oxygen atmosphere at $23{ }^{\circ} \mathrm{C}$ for 12 hours, when TLC analysis indicated consumption of starting material. The solvent was removed under reduced pressure, and the residue was loaded onto a short plug of silica gel, eluting with $30 \%$ ethyl acetate in hexanes (100 $\mathrm{mL}$ ). The oil obtained upon concentration was then redissolved in THF ( $4 \mathrm{~mL}$ total volume) and treated with benzylamine $\left(23 \mu \mathrm{L}, 0.21 \mathrm{mmol}, 1.05\right.$ equiv) at $23{ }^{\circ} \mathrm{C}$. After one hour, trimethylsilyl cyanide ( $26 \mu \mathrm{L}, 0.21 \mathrm{mmol}, 1.05$ equiv) was added, and the resulting mixture was stirred at $23{ }^{\circ} \mathrm{C}$ for 7 hours, at which time the volatiles were removed under reduced pressure. The crude residue obtained was purified by silica gel column chromatography ( $20 \%$ ethyl acetate in hexanes) to furnish $\alpha$-aminonitrile $\mathbf{8}$ as a colorless oil (59.6 mg, 86\% yield). $\mathrm{R} f=0.42$ (33\% ethyl acetate in hexanes); ${ }^{1} \mathrm{H}$ NMR $\left(\mathrm{CDCl}_{3}, 500 \mathrm{MHz}\right) \delta 7.37-7.31(\mathrm{~m}, 4 \mathrm{H}), 7.31-7.26(\mathrm{~m}, 1 \mathrm{H}), 4.18(\mathrm{qd}, J=7.1,2.2 \mathrm{~Hz}$, $4 \mathrm{H}), 4.06(\mathrm{~d}, J=12.9 \mathrm{~Hz}, 1 \mathrm{H}), 3.82(\mathrm{~d}, J=12.9 \mathrm{~Hz}, 1 \mathrm{H}), 3.49$ (t, $J=7.0 \mathrm{~Hz}, 1 \mathrm{H}), 2.17-$ $2.05(\mathrm{~m}, 1 \mathrm{H}), 2.00(\mathrm{ddd}, J=13.7,9.5,7.4 \mathrm{~Hz}, 1 \mathrm{H}), 1.81-1.73(\mathrm{~m}, 2 \mathrm{H}), 1.41(\mathrm{~s}, 3 \mathrm{H}), 1.24$ $(\mathrm{td}, J=7.1,2.3 \mathrm{~Hz}, 6 \mathrm{H}) ;{ }^{13} \mathrm{C} \mathrm{NMR}\left(\mathrm{CDCl}_{3}, 126 \mathrm{MHz}\right) \delta 171.9,138.2,128.7,128.5$, 127.7, 119.8, 61.6, 53.2, 51.7, 49.8, 31.8, 28.9, 20.2, 14.2; IR (Neat Film, KBr) 3325, 2983, 1728, 1454, 1261, 1189, 1112, 1027, 738, $700 \mathrm{~cm}^{-1}$; HRMS (ESI+) $m / z$ calc'd for $\mathrm{C}_{19} \mathrm{H}_{27} \mathrm{~N}_{2} \mathrm{O}_{4}[\mathrm{M}+\mathrm{H}]^{+}: 347.1965$, found 347.1970. 


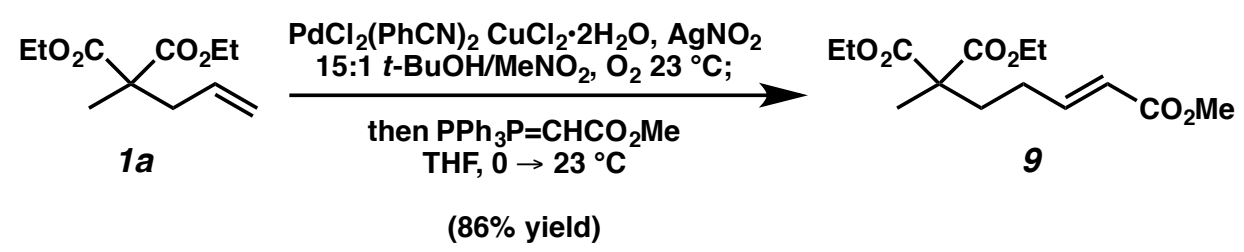

5,5-Diethyl 1-methyl $(E)$-hex-1-ene-1,5,5-tricarboxylate (9):

To a flame-dried $25-\mathrm{mL}$ round-bottom flask with a magnetic stir bar were added bis(benzonitrile)palladium(II) chloride $(9.2 \mathrm{mg}, 0.024 \mathrm{mmol}, 0.12$ equiv), copper(II) chloride dihydrate $(4.1 \mathrm{mg}, 0.024 \mathrm{mmol}, 0.12$ equiv), and silver nitrite $(1.8 \mathrm{mg}, 0.012$ mmol, 0.06 equiv). The flask was capped with a rubber septum, and tert-butyl alcohol $(3.75 \mathrm{~mL})$ and nitromethane $(0.25 \mathrm{~mL})$ were added sequentially by syringe. The mixture was stirred at $23{ }^{\circ} \mathrm{C}$ and sparged with oxygen gas (balloon) for 3 minutes. Alkene 1a (42.9 $\mathrm{mg}, 0.20 \mathrm{mmol}, 1.00$ equiv) was added dropwise by syringe, and the reaction mixture was sparged with oxygen for another minute. The reaction was stirred under oxygen atmosphere at $23{ }^{\circ} \mathrm{C}$ for 12 hours, when TLC analysis indicated consumption of starting material. The solvent was removed under reduced pressure, and the residue was loaded onto a short plug of silica gel, eluting with 30\% ethyl acetate in hexanes (100 $\mathrm{mL}$ ). The oil obtained upon concentration was then redissolved in THF (4 mL total volume) and cooled to $0{ }^{\circ} \mathrm{C}$ using an ice water bath. Carbomethoxy methylene triphenyl phosphorane (100.3 mg, $0.30 \mathrm{mmol}, 1.50$ equiv) was added in one portion, and the resulting mixture was stirred at $23{ }^{\circ} \mathrm{C}$ for 20 hours, at which time the reaction was transferred to a separatory funnel with diethyl ether and washed sequentially with water $(5 \mathrm{~mL})$ and brine $(5 \mathrm{~mL})$. The organic layer was dried over sodium sulfate, filtered, and concentrated to a crude yellow oil. Purification by silica gel column chromatography (10\% ethyl acetate in hexanes) afforded $\alpha, \beta$-unsaturated methyl ester 9 as a colorless oil (49.3 mg, 86\% yield). $\mathrm{R} f=0.56$ (33\% ethyl acetate in hexanes); ${ }^{1} \mathrm{H} \mathrm{NMR}\left(\mathrm{CDCl}_{3}, 300\right.$ MHz) $\delta 6.93$ (dtd, $J=15.3,6.7,1.8 \mathrm{~Hz}, 1 \mathrm{H}$ ), 5.83 (dt, $J=15.7,1.7 \mathrm{~Hz}, 1 \mathrm{H}), 4.17$ (qd, $J=$ $7.2,1.7 \mathrm{~Hz}, 4 \mathrm{H}), 3.71(\mathrm{~d}, J=1.9 \mathrm{~Hz}, 3 \mathrm{H}), 2.26-2.10(\mathrm{~m}, 2 \mathrm{H}), 2.04-1.92(\mathrm{~m}, 2 \mathrm{H}), 1.41(\mathrm{~d}$, $J=1.7 \mathrm{~Hz}, 3 \mathrm{H}), 1.24(\mathrm{td}, J=7.1,1.7 \mathrm{~Hz}, 6 \mathrm{H}) ;{ }^{13} \mathrm{C} \mathrm{NMR}\left(\mathrm{CDCl}_{3}, 126 \mathrm{MHz}\right) \delta 172.0$, 167.0, 148.1, 121.5, 61.5, 53.4, 51.6, 33.9, 27.3, 20.1, 14.2; IR (Neat Film, KBr) 2984, 2951, 1734, 1730, 1659, 1437, 1268, 1234, 1110, 1024, $858 \mathrm{~cm}^{-1}$; HRMS (FAB+) $\mathrm{m} / \mathrm{z}$ calc'd for $\mathrm{C}_{14} \mathrm{H}_{23} \mathrm{O}_{6}[\mathrm{M}+\mathrm{H}]^{+}:$287.1489, found 287.1485.

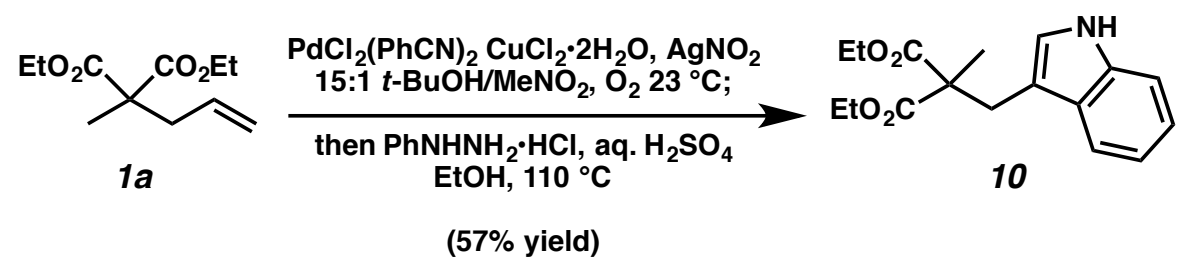

Diethyl 2-((1H-indol-3-yl)methyl)-2-methylmalonate (10):

To a flame-dried $25-\mathrm{mL}$ round-bottom flask with a magnetic stir bar were added bis(benzonitrile)palladium(II) chloride ( $23.0 \mathrm{mg}, 0.060 \mathrm{mmol}, 0.12$ equiv), copper(II) chloride dihydrate ( $10.2 \mathrm{mg}, 0.060 \mathrm{mmol}, 0.12$ equiv), and silver nitrite ( $4.6 \mathrm{mg}, 0.030$ mmol, 0.06 equiv). The flask was capped with a rubber septum, and tert-butyl alcohol $(9.4 \mathrm{~mL})$ and nitromethane $(0.60 \mathrm{~mL})$ were added sequentially by syringe. The mixture was stirred at $23{ }^{\circ} \mathrm{C}$ and sparged with oxygen gas (balloon) for 3 minutes. Alkene 1a 
(107 mg, $0.50 \mathrm{mmol}, 1.00$ equiv) was added dropwise by syringe, and the reaction mixture was sparged with oxygen for another minute. The reaction was stirred under oxygen atmosphere at $23{ }^{\circ} \mathrm{C}$ for 12 hours, when TLC analysis indicated consumption of starting material. The solvent was removed under reduced pressure, and the residue was loaded onto a short plug of silica gel, eluting with $30 \%$ ethyl acetate in hexanes (100 $\mathrm{mL})$. The oil obtained upon concentration was then diluted with a pre-heated solution $\left(50{ }^{\circ} \mathrm{C}\right)$ of $4 \%$ aqueous sulfuric acid $(4.7 \mathrm{~mL})$ and phenyl hydrazine hydrochloride $(79.5$ $\mathrm{mg}, 0.550 \mathrm{mmol}, 1.10$ equiv). After addition of ethanol $(3.5 \mathrm{~mL})$, the mixture was heated to reflux at $110^{\circ} \mathrm{C}$ for 7 hours. The reaction mixture was cooled to $23{ }^{\circ} \mathrm{C}$ and treated with saturated aqueous sodium bicarbonate and ethyl acetate. The phases were separated, and the aqueous layer was extracted with ethyl acetate $(2 \times 20 \mathrm{~mL})$. The combined organic extracts were dried over sodium sulfate before filtration and concentration under reduced pressure. The crude residue was purified by silica gel column chromatography (20\% ethyl acetate in hexanes) to afford indole $\mathbf{1 0}$ as yellow oil (86.1 mg, $57 \%$ yield). $\mathrm{R} f$ $=0.44(33 \%$ ethyl acetate in hexanes $) ;{ }^{1} \mathrm{H} \mathrm{NMR}\left(\mathrm{CDCl}_{3}, 500 \mathrm{MHz}\right) \delta 8.17(\mathrm{~s}, 1 \mathrm{H}), 7.59$ $(\mathrm{d}, J=7.9 \mathrm{~Hz}, 1 \mathrm{H}), 7.32(\mathrm{dt}, J=8.1,1.0 \mathrm{~Hz}, 1 \mathrm{H}), 7.11(\mathrm{ddd}, J=8.0,7.0,1.1 \mathrm{~Hz}, 1 \mathrm{H})$, $6.98(\mathrm{~d}, J=2.4 \mathrm{~Hz}, 1 \mathrm{H}), 6.98(\mathrm{~d}, J=2.4 \mathrm{~Hz}, 1 \mathrm{H}), 4.27-4.10(\mathrm{~m}, 4 \mathrm{H}), 3.41(\mathrm{~d}, J=0.9 \mathrm{~Hz}$, $2 \mathrm{H}), 1.44(\mathrm{~s}, 3 \mathrm{H}), 1.24(\mathrm{t}, J=7.1 \mathrm{~Hz}, 6 \mathrm{H}) ;{ }^{13} \mathrm{C} \mathrm{NMR}\left(\mathrm{CDCl}_{3}, 126 \mathrm{MHz}\right) \delta 172.6,135.9$, $128.5,123.5,121.9,119.5,111.2,110.5,61.4,55.4,30.7,20.4,14.1$; IR (Neat Film, KBr) 3403, 2983, 1728, 1458, 1293, 1254, 1106, 1021, 861, $743 \mathrm{~cm}^{-1}$; HRMS (ESI+) $\mathrm{m} / z$ calc'd for $\mathrm{C}_{17} \mathrm{H}_{22} \mathrm{NO}_{4}[\mathrm{M}+\mathrm{H}]^{+}: 304.1543$, found 304.1548 .
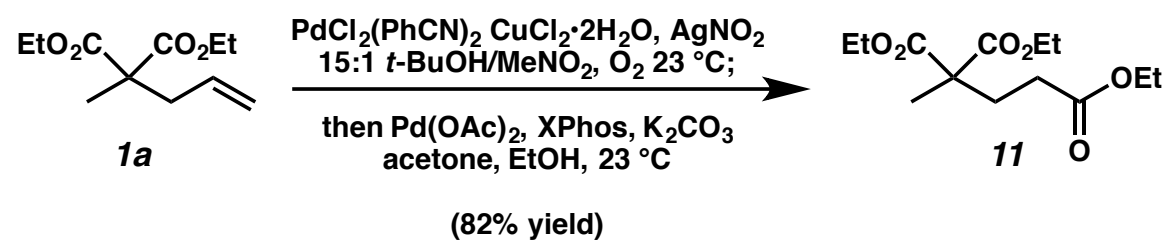

Triethyl butane-1,3,3-tricarboxylate (11):

To a flame-dried 25-mL round-bottom flask with a magnetic stir bar were added bis(benzonitrile)palladium(II) chloride $(9.2 \mathrm{mg}, 0.024 \mathrm{mmol}, 0.12$ equiv), copper(II) chloride dihydrate ( $4.1 \mathrm{mg}, 0.024 \mathrm{mmol}, 0.12$ equiv), and silver nitrite (1.8 $\mathrm{mg}, 0.012$ mmol, 0.06 equiv). The flask was capped with a rubber septum, and tert-butyl alcohol $(3.75 \mathrm{~mL})$ and nitromethane $(0.25 \mathrm{~mL})$ were added sequentially by syringe. The mixture was stirred at $23{ }^{\circ} \mathrm{C}$ and sparged with oxygen gas (balloon) for 3 minutes. Alkene $\mathbf{1 a}$ (42.9 mg, $0.20 \mathrm{mmol}, 1.00$ equiv) was added dropwise by syringe, and the reaction mixture was sparged with oxygen for another minute. The reaction was stirred under oxygen atmosphere at $23{ }^{\circ} \mathrm{C}$ for 12 hours, when TLC analysis indicated consumption of starting material. The solvent was removed under reduced pressure, and the residue was loaded onto a short plug of silica gel, eluting with 30\% ethyl acetate in hexanes (100 $\mathrm{mL})$. The oil obtained upon concentration was then redissolved in degassed ethanol (2 $\mathrm{mL})$, and oven-dried potassium carbonate $(10.0 \mathrm{mg}, 0.072 \mathrm{mmol}, 0.36$ equiv) was added. After stirring for 20 minutes, a solution of palladium(II) acetate $(2.2 \mathrm{mg}, 0.01 \mathrm{mmol}, 0.05$ equiv) and XPhos (9.5 mg, $0.02 \mathrm{mmol}, 0.10$ equiv) in acetone $(2 \mathrm{~mL})$ that had been stirring at $23{ }^{\circ} \mathrm{C}$ for 20 minutes was added via syringe under argon atmosphere. The resulting dark green solution was stirred at $23{ }^{\circ} \mathrm{C}$ for 6 hours, at which time the volatiles 
were removed under reduced pressure. The crude residue obtained was purified by silica gel column chromatography (8\% ethyl acetate in hexanes) to furnish tri-ester $\mathbf{1 1}$ as a colorless oil (45.0 mg, 82\% yield). $\mathrm{R} f=0.53$ (33\% ethyl acetate in hexanes); ${ }^{1} \mathrm{H}$ NMR $\left(\mathrm{CDCl}_{3}, 500 \mathrm{MHz}\right) \delta 4.16(\mathrm{q}, J=7.1,0.8 \mathrm{~Hz}, 4 \mathrm{H}), 4.11(\mathrm{q}, J=7.2,0.9 \mathrm{~Hz}, 2 \mathrm{H}), 2.38-$ $2.27(\mathrm{~m}, 2 \mathrm{H}), 2.23-2.13(\mathrm{~m}, 2 \mathrm{H}), 1.39(\mathrm{~s}, 3 \mathrm{H}), 1.23(\mathrm{td}, J=7.1,0.8 \mathrm{~Hz}, 9 \mathrm{H}) ;{ }^{13} \mathrm{C} \mathrm{NMR}$ $\left(\mathrm{CDCl}_{3}, 126 \mathrm{MHz}\right) \delta 173.0,171.9,61.5,60.6,53.0,30.7,29.9,20.2,14.3$; IR (Neat Film, $\mathrm{KBr}) 2982,2941,1738,1732,1466,1380,1243,1185,1109,1025,860 \mathrm{~cm}^{-1}$; HRMS (ESI+) $m / z$ calc'd for $\mathrm{C}_{13} \mathrm{H}_{23} \mathrm{O}_{6}[\mathrm{M}+\mathrm{H}]^{+}: 275.1489$, found 275.1483 .

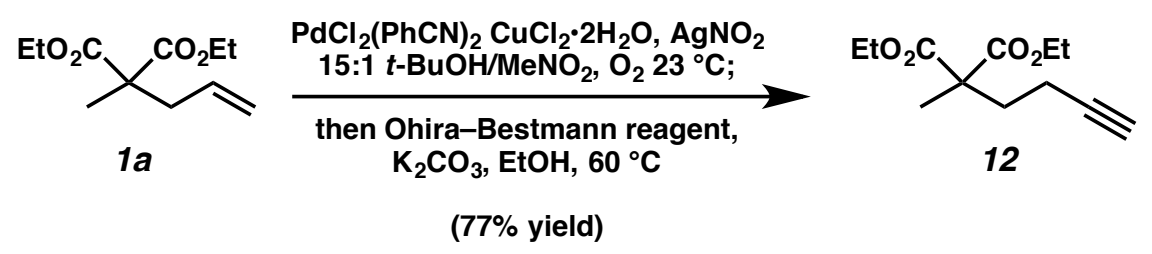

Diethyl 2-(but-3-yn-1-yl)-2-methylmalonate (12):

To a flame-dried $25-\mathrm{mL}$ round-bottom flask with a magnetic stir bar were added bis(benzonitrile)palladium(II) chloride $(9.2 \mathrm{mg}, 0.024 \mathrm{mmol}, 0.12$ equiv), copper(II) chloride dihydrate $(4.1 \mathrm{mg}, 0.024 \mathrm{mmol}, 0.12$ equiv), and silver nitrite $(1.8 \mathrm{mg}, 0.012$ mmol, 0.06 equiv). The flask was capped with a rubber septum, and tert-butyl alcohol $(3.75 \mathrm{~mL})$ and nitromethane $(0.25 \mathrm{~mL})$ were added sequentially by syringe. The mixture was stirred at $23{ }^{\circ} \mathrm{C}$ and sparged with oxygen gas (balloon) for 3 minutes. Alkene $\mathbf{1 a}$ (42.9 mg, $0.20 \mathrm{mmol}, 1.00$ equiv) was added dropwise by syringe, and the reaction mixture was sparged with oxygen for another minute. The reaction was stirred under oxygen atmosphere at $23{ }^{\circ} \mathrm{C}$ for 12 hours, when TLC analysis indicated consumption of starting material. The solvent was removed under reduced pressure, and the residue was loaded onto a short plug of silica gel, eluting with 30\% ethyl acetate in hexanes (100 $\mathrm{mL})$. The oil obtained upon concentration was then redissolved in ethanol (4 mL), and potassium carbonate $(33.2 \mathrm{mg}, 0.24 \mathrm{mmol}, 1.20$ equiv) and Ohira-Bestmann reagent (46.1 mg, $0.24 \mathrm{mmol}, 1.20$ equiv) were added. The resulting mixture was stirred at $60{ }^{\circ} \mathrm{C}$ for 24 hours, at which time the reaction was quenched with water $(4 \mathrm{~mL})$, diluted with diethyl ether $(2 \mathrm{~mL})$, and washed with $5 \%$ aqueous sodium bicarbonate. The organic layer was dried over magnesium sulfate, filtered, and concentrated. The crude residue obtained was purified by silica gel column chromatography ( $8 \%$ ethyl acetate in hexanes) to furnish alkyne 12 as a colorless oil $(35.0 \mathrm{mg}, 77 \%$ yield). $\mathrm{R} f=0.72$ (33\% ethyl acetate in hexanes); ${ }^{1} \mathrm{H} \mathrm{NMR}\left(\mathrm{CDCl}_{3}, 300 \mathrm{MHz}\right) \delta 4.18(\mathrm{q}, J=7.1 \mathrm{~Hz}, 4 \mathrm{H}), 2.28-2.06(\mathrm{~m}, 4 \mathrm{H})$, $1.95(\mathrm{t}, J=2.5 \mathrm{~Hz}, 1 \mathrm{H}), 1.42(\mathrm{~s}, 3 \mathrm{H}), 1.25(\mathrm{t}, J=7.1 \mathrm{~Hz}, 7 \mathrm{H}) ;{ }^{13} \mathrm{C} \mathrm{NMR}\left(\mathrm{CDCl}_{3}, 75\right.$ $\mathrm{MHz}) \delta 171.9,83.5,68.8,61.5,53.2,34.6,20.0$, 14.3, 14.2; IR (Neat Film, KBr) 3291, 2983, 1731, 1465, 1381, 1265, 1189, 1109, 1025, 861, $659 \mathrm{~cm}^{-1}$; HRMS (FAB+) $\mathrm{m} / z$ calc'd for $\mathrm{C}_{12} \mathrm{H}_{20} \mathrm{O}_{4}[\mathrm{M}+\mathrm{H}]^{+}:$227.1283, found 227.1287 . 


\section{Notes and References}

1. Pangborn, A. B.; Giardello, M. A.; Grubbs, R. H.; Rosen, R. K.; Timmers, F. J. Organometallics 1996, 15, 1518-1520.

2. Pietruszka, J.; Witt, A. Synthesis 2006, 24, 4266-4268.

3. Boers, R. B.; Randulfe, Y. P.; van der Haas, H. N. S.; van Rossum-Baan, M.; Lugtenburg, J. Eur. J. Org. Chem. 2002, 2094-2108.

4. Anhydrous $\mathrm{CuCl}$ and $\mathrm{CuCl}_{2}$ were also examined as copper sources, but use of $\mathrm{CuCl}_{2} \cdot 2 \mathrm{H}_{2} \mathrm{O}$ resulted in the highest yields.

5. While investigations into the complex mechanism of this transformation are still ongoing, evidence suggests that a $t$ - $\mathrm{BuOH}$-ligated nitrite $\mathrm{Pd}-\mathrm{Cu}$ species promotes selective formation of the aldehyde. For more mechanistic analysis, see: a) Jiang, Y.-Y.; Zhang, Q.; Yu, H.-Z.; Fu, Y. ACS Catal. 2015, 5, 1414-1423; b)

Anderson, B. J.; Keith, J. A.; Sigman, M. S. J. Am. Chem. Soc. 2010, 132, 1187211874; c) Keith, J. A.; Nielsen, R. J.; Oxgaard, J.; Goddard, W. A., III J. Am. Chem. Soc. 2007, 129, 12342-12343.

6. Lactam substrates bearing quaternary carbons at the homoallylic position were also investigated, but these substrates reacted sluggishly, and only low yields (32$37 \%$ ) of the aldehyde product were obtained, often contaminated by enal side product.

7. Xing, X.; O’Connor, N. R.; Stoltz, B. M. Angew. Chem., Int. Ed. 2015, 54, $11186-11190$.

8. Hong, A. Y.; Krout, M. R.; Jensen, T.; Bennett, N. B.; Harned, A. M.; Stoltz, B. M. Angew. Chem., Int. Ed. 2011, 50, 2756-2760.

9. Behenna, D. C.; Mohr, J. T.; Sherden, N. H.; Marinescu, S. C.; Harned, A. M.; Tani, K.; Seto, M.; Ma, S.; Novák, Z.; Krout, M. R.; McFadden, R. M.; Roizen, J. L.; Enquist, J. A., Jr.; White, D. E.; Levine, S. R.; Petrova, K. V.; Iwashita, A.; Virgil, S. C.; Stoltz, B. M. Chem. - Eur. J. 2011, 17, 14199-14223.

10. Liu, W.-B.; Reeves, C. M.; Virgil, S. C.; Stoltz, B. M. J. Am. Chem. Soc. 2013, 135, 10626-10629.

11. Liu, Y.; Virgil, S. C.; Grubbs, R. H.; Stoltz, B. M. Angew. Chem., Int. Ed. 2015, $54,11800-11803$.

12. Zhang, X.; Jia, X.; Fang, L.; Liu, N.; Wang, J.; Fan, X. Org. Lett. 2011, 13, 50245027.

13. Ghorai, M. K.; Talukdar, R.; Tiwari, D. P. Org. Lett. 2014, 16, 2204-2207.

14. When unprotected 1d was subjected to the aldehyde-selective Wacker conditions, mixtures containing several inseparable compounds were obtained after purification. 


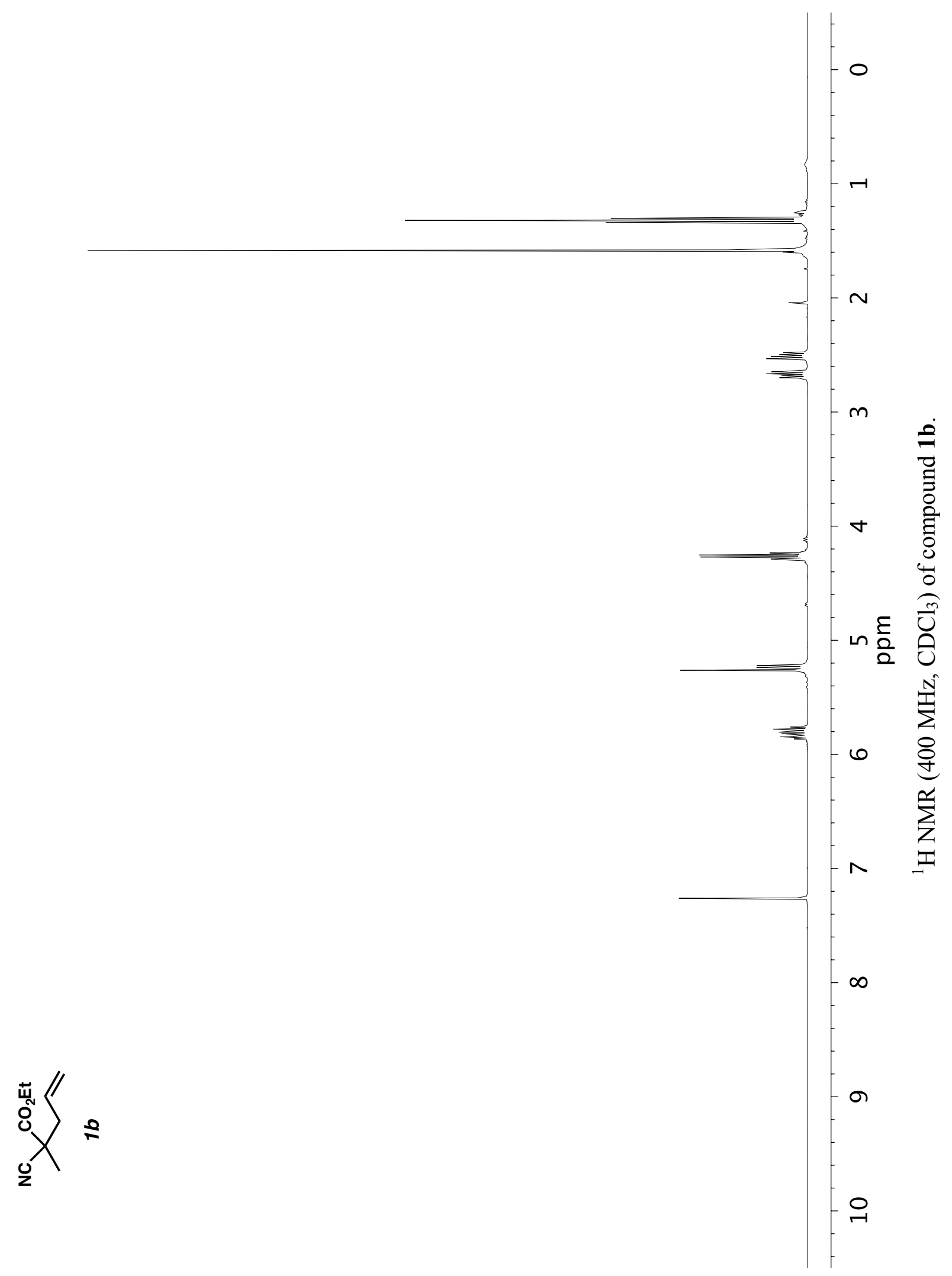




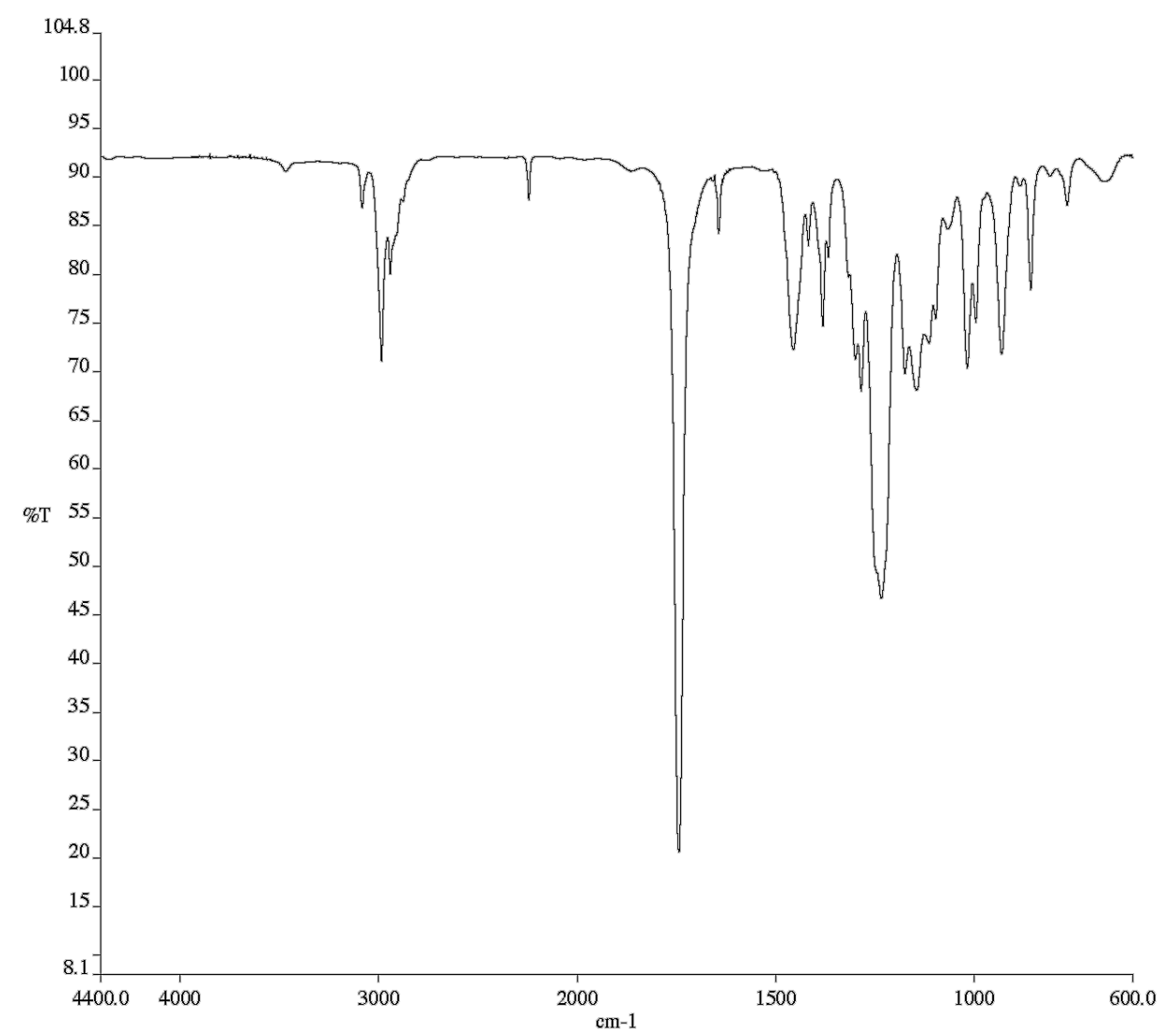

Infrared spectrum (Thin Film, KBr) of compound $\mathbf{1 b}$.

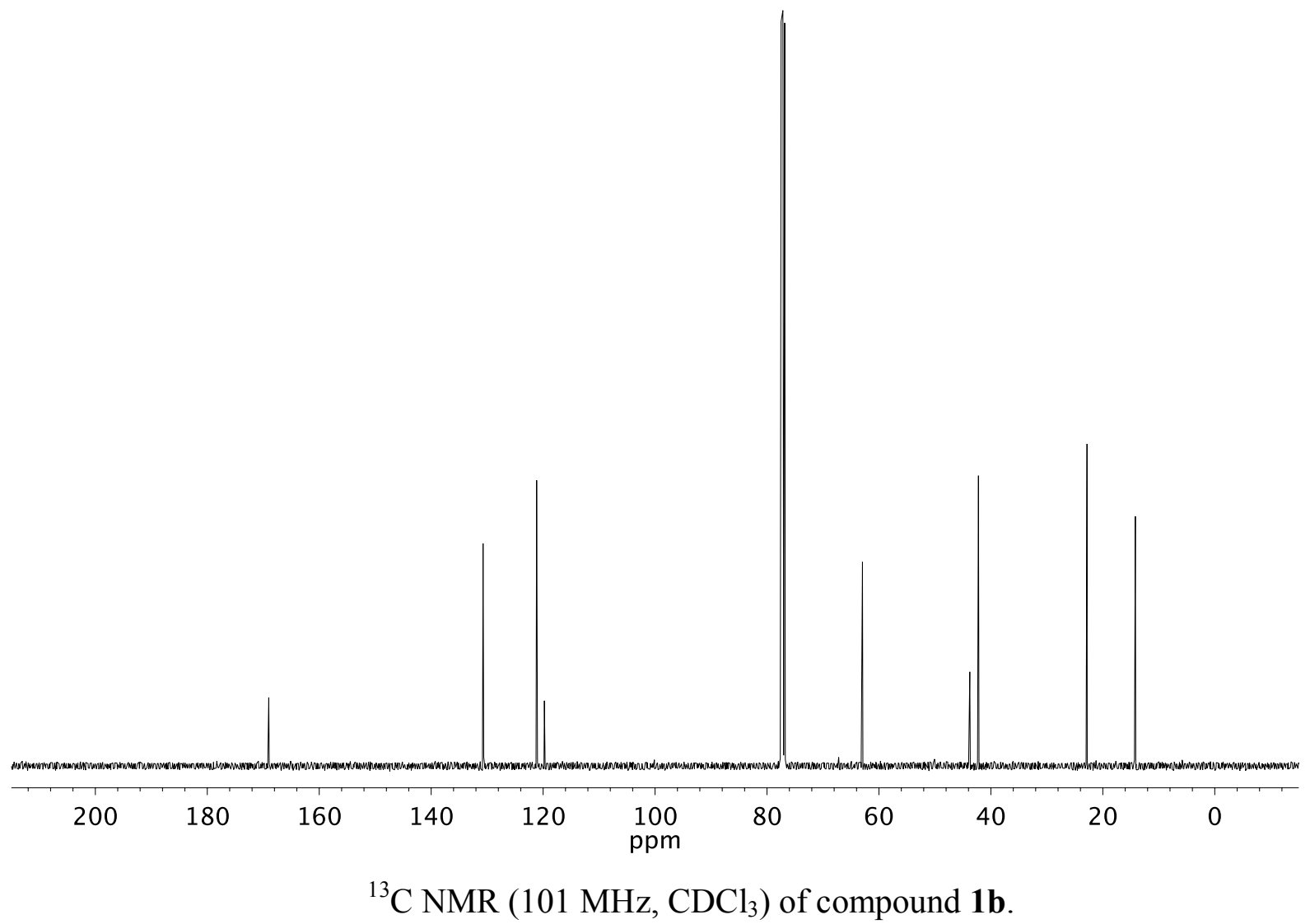




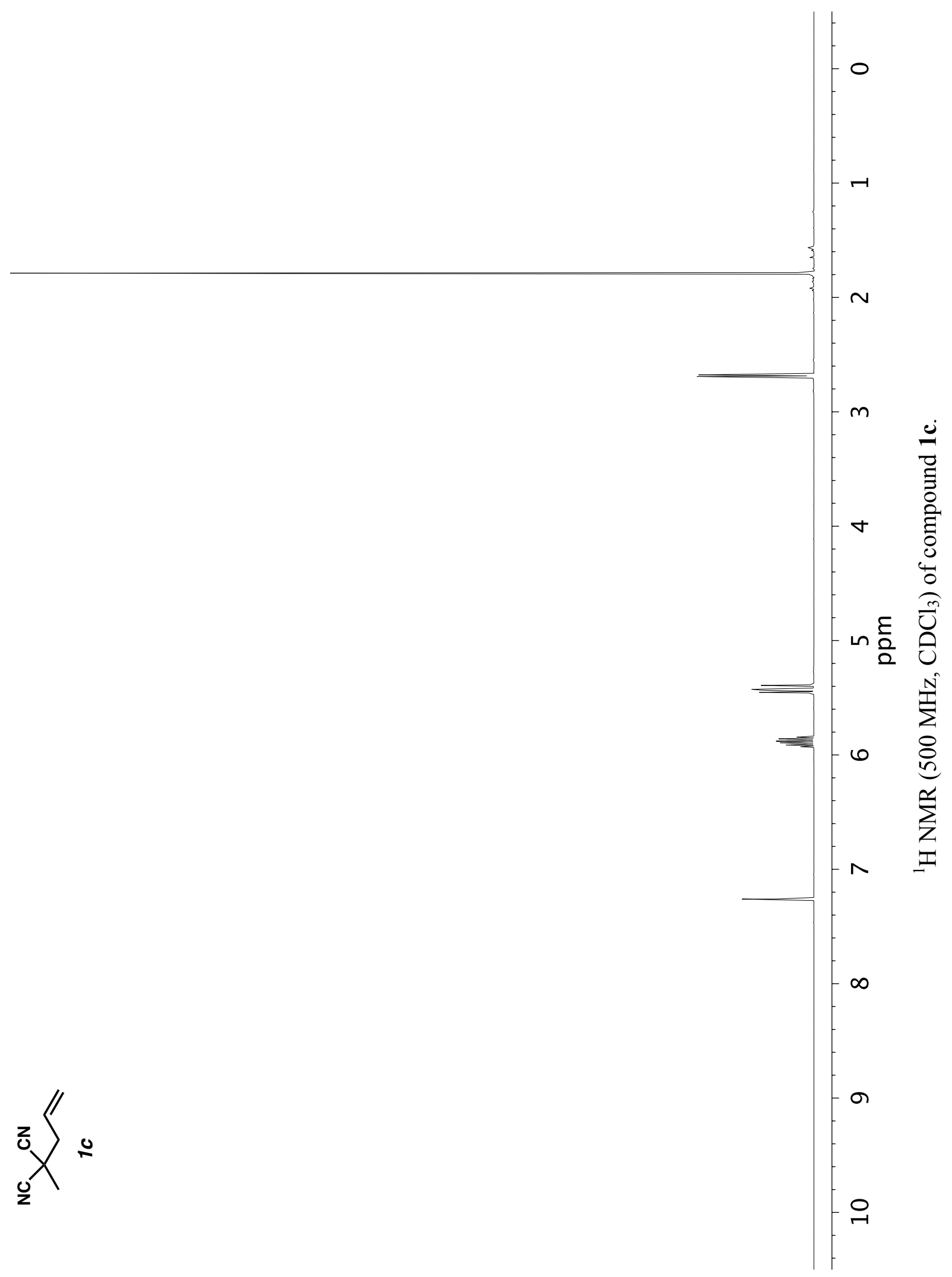



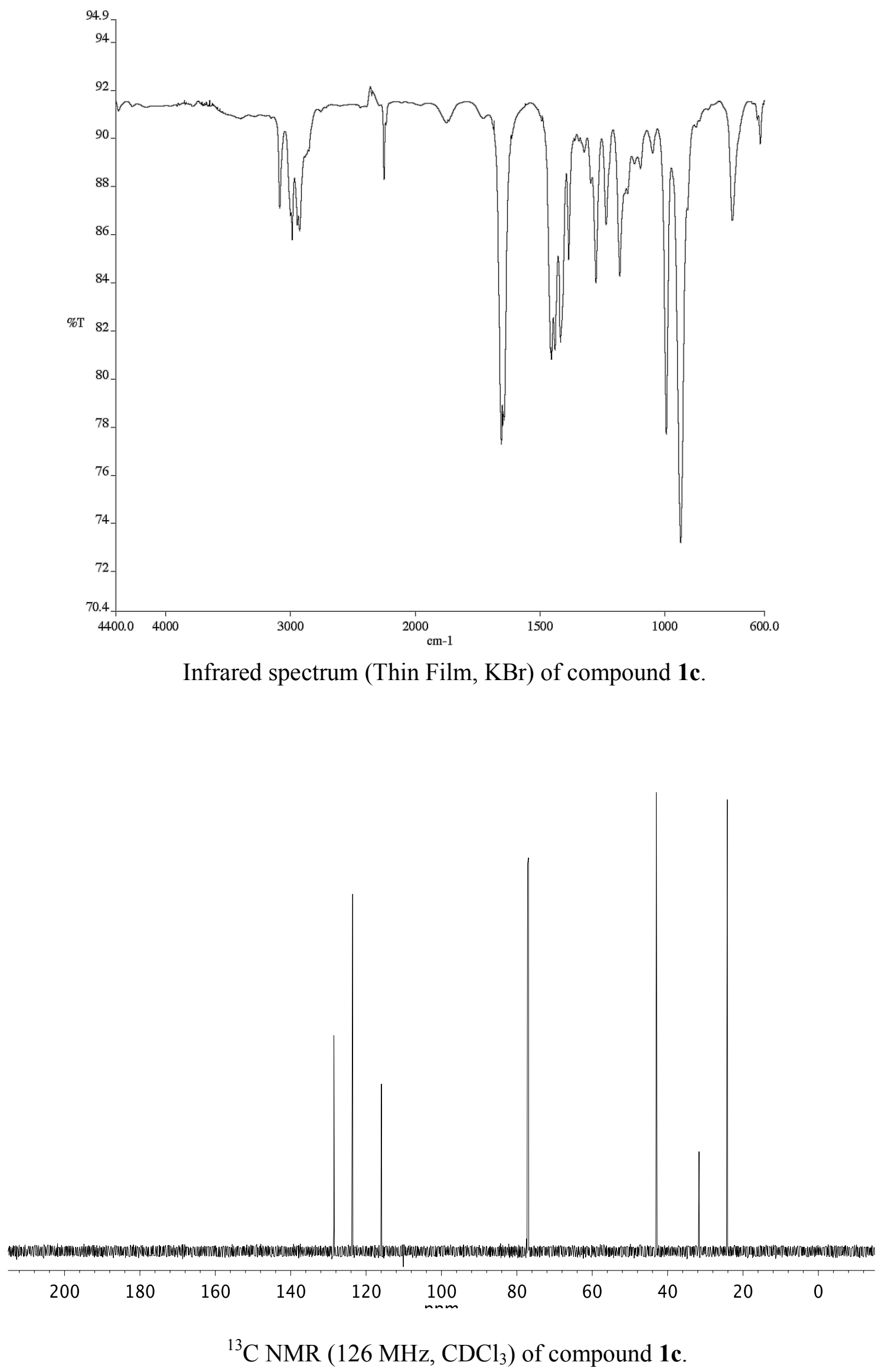


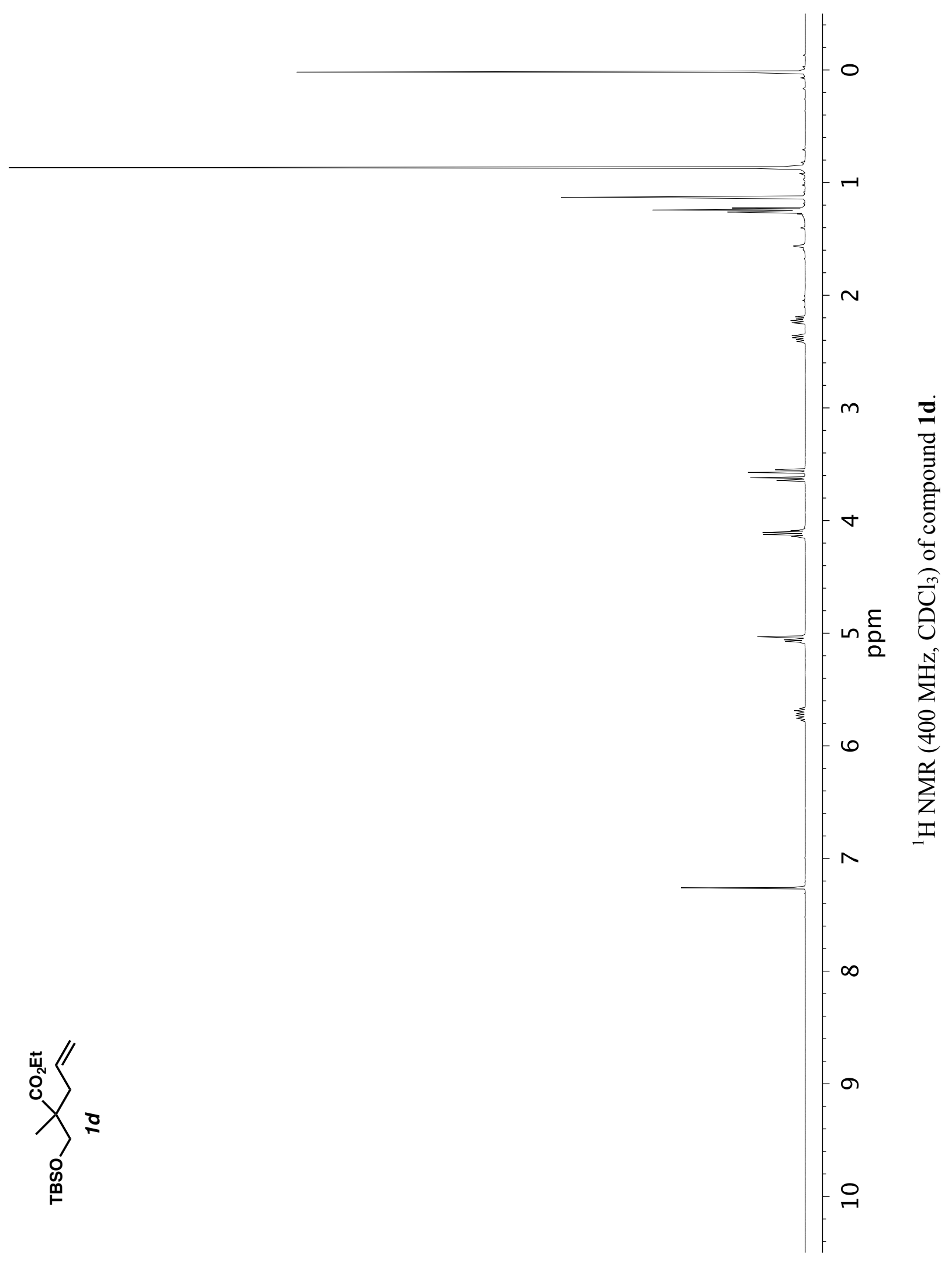



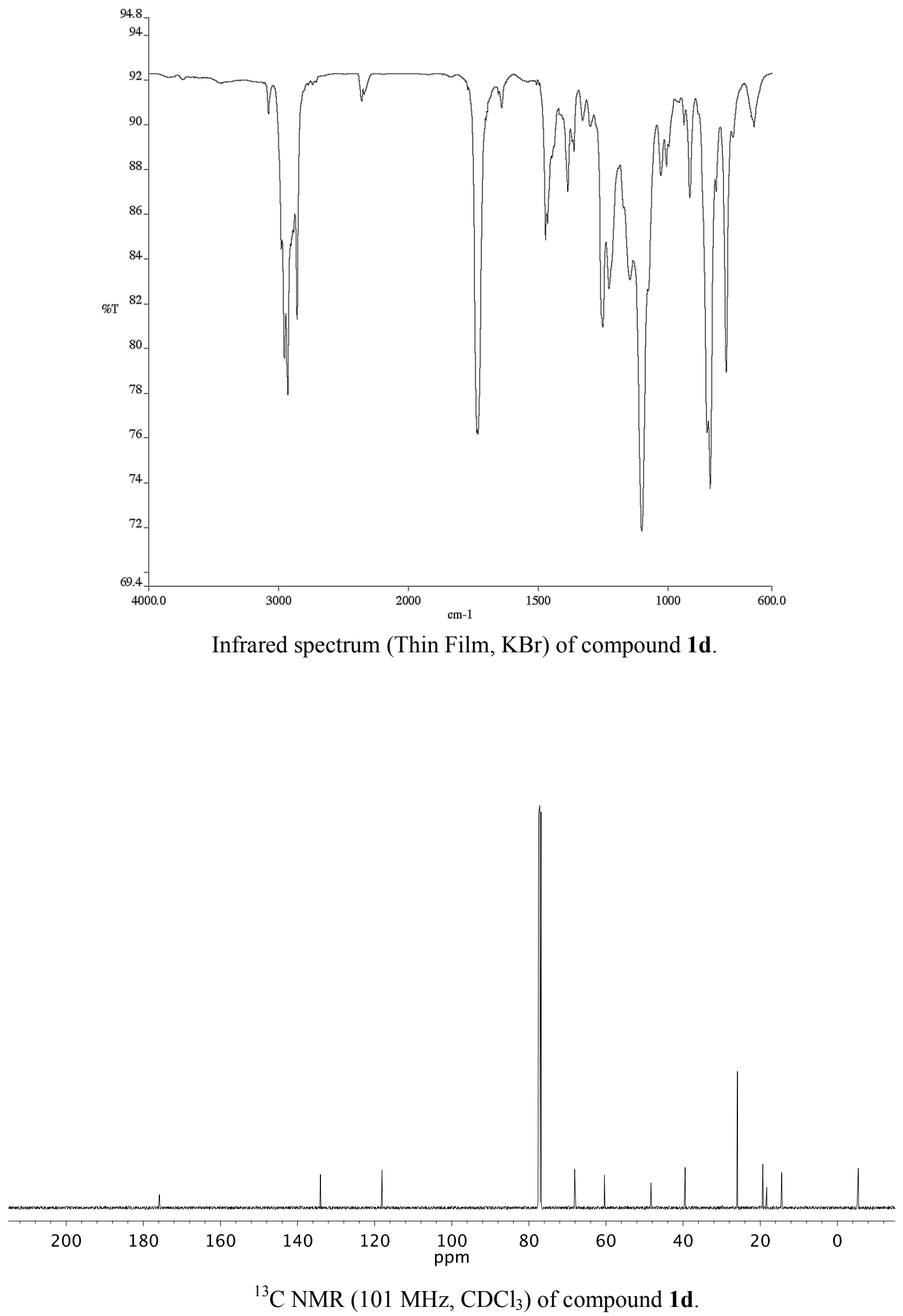


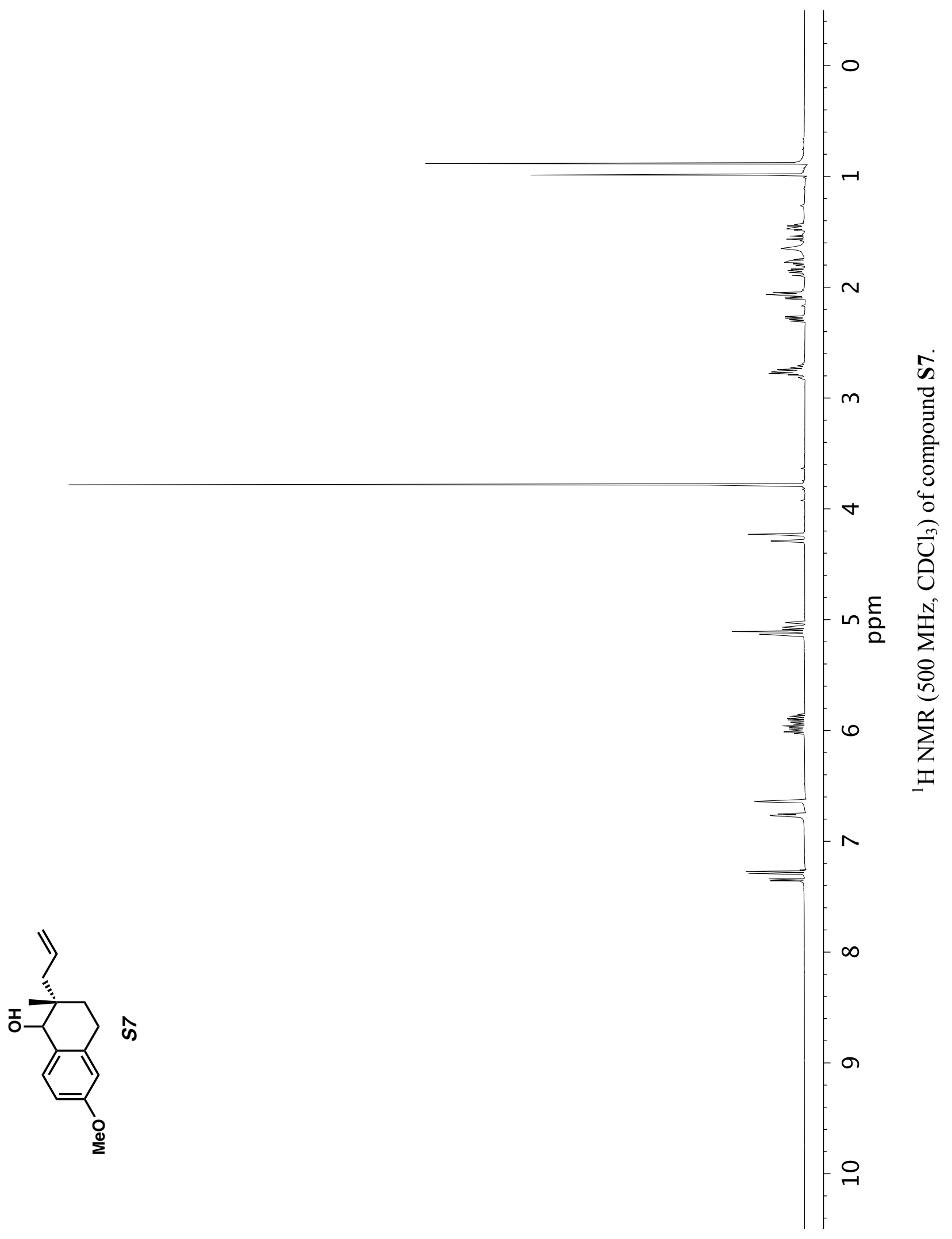



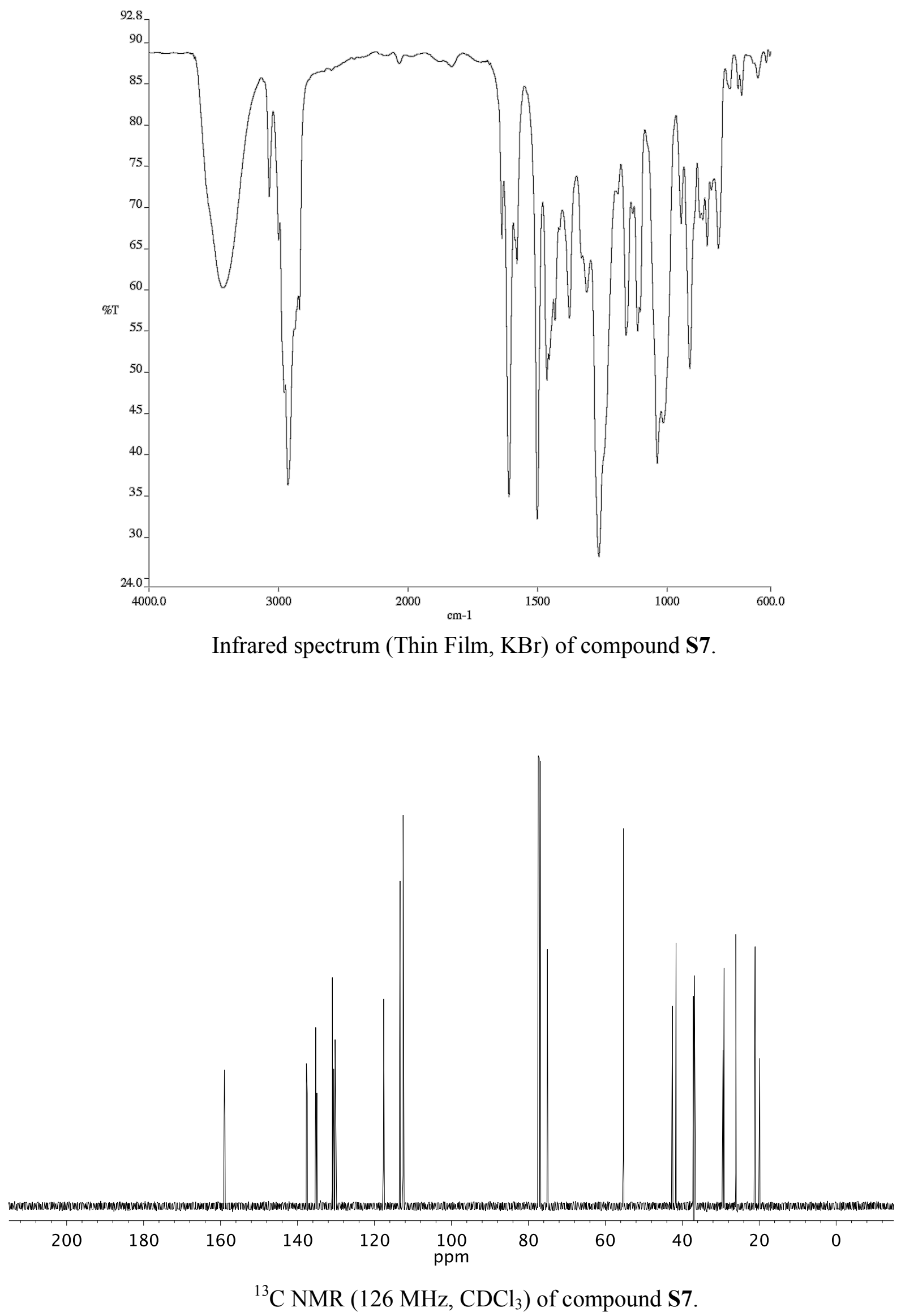


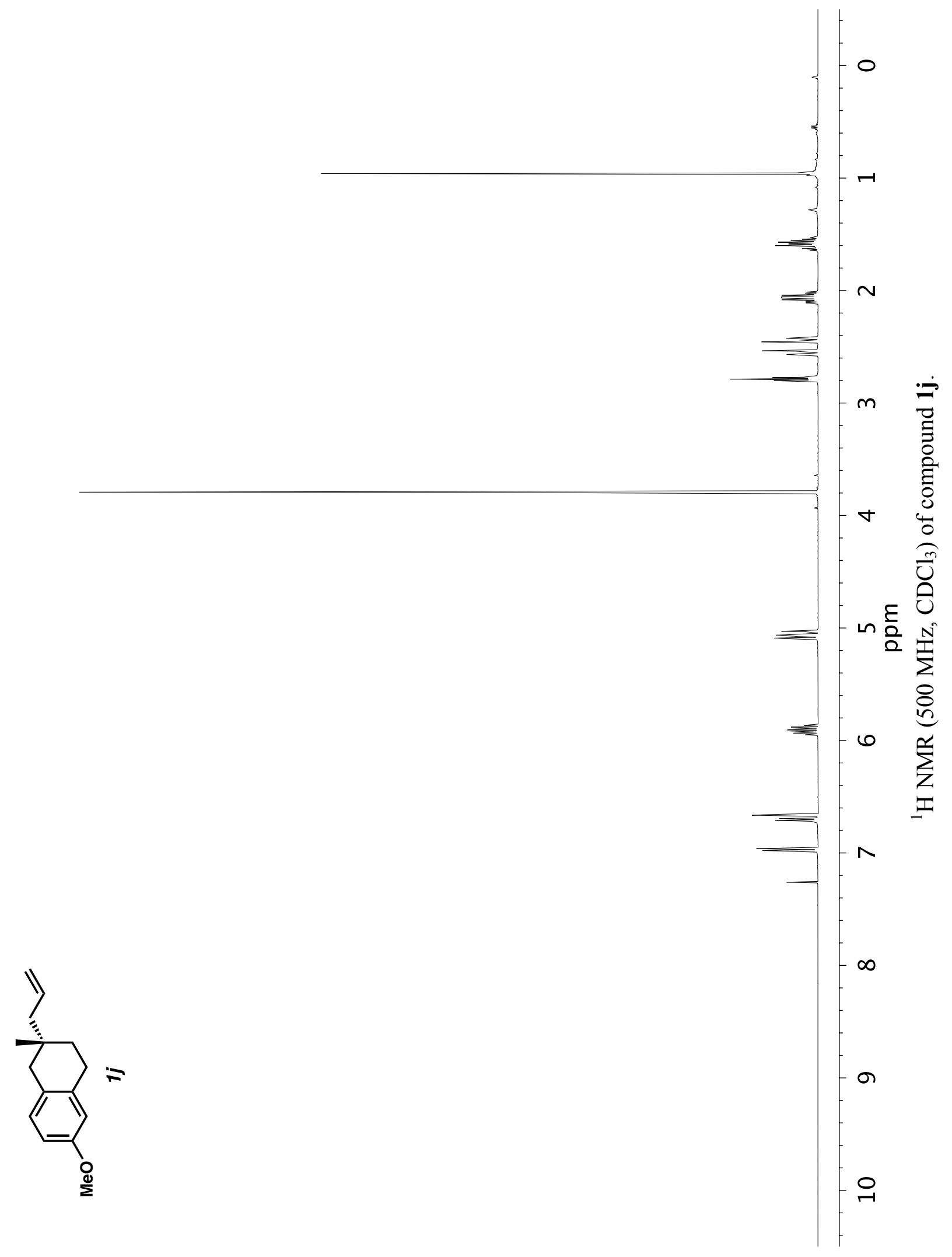



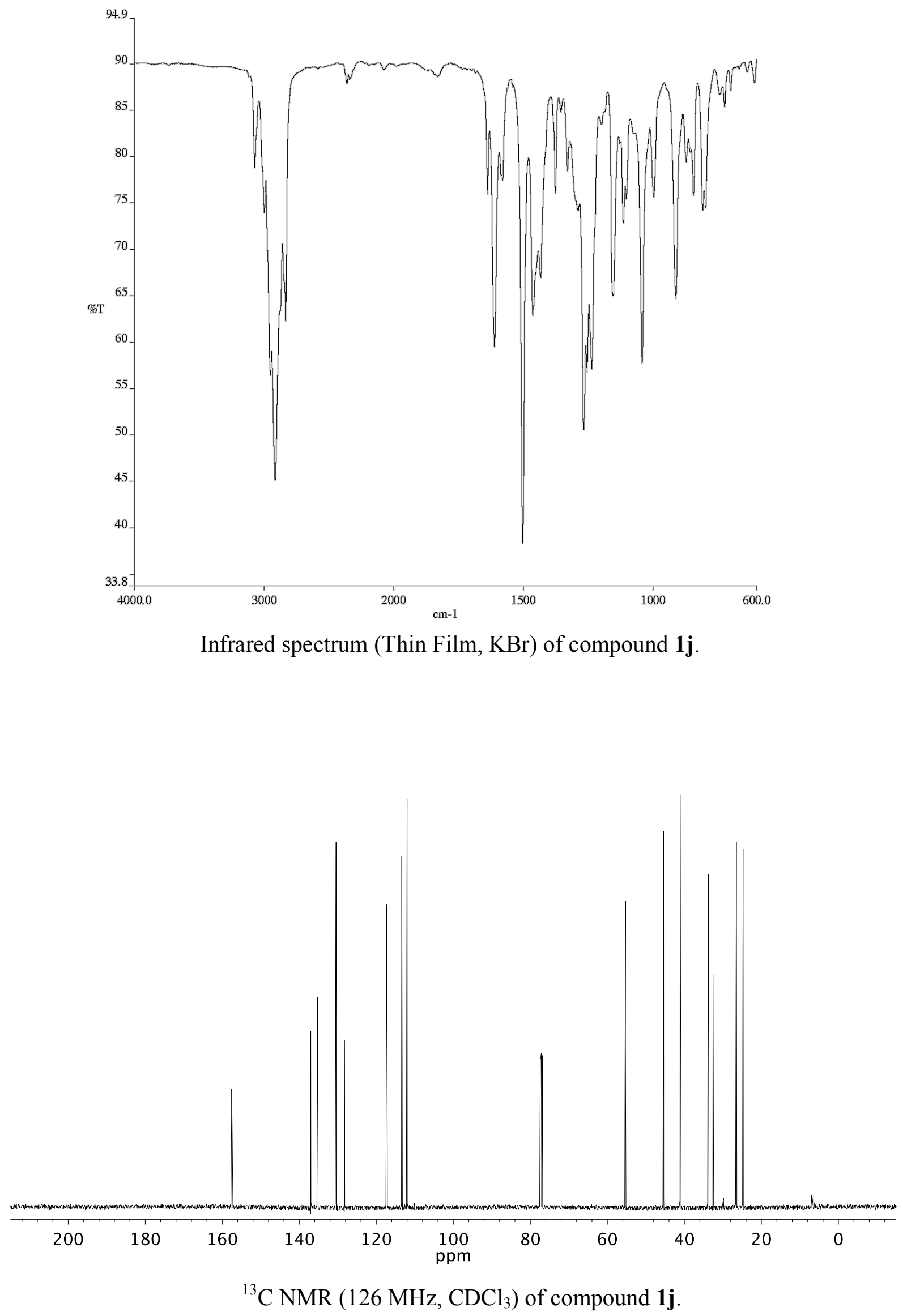


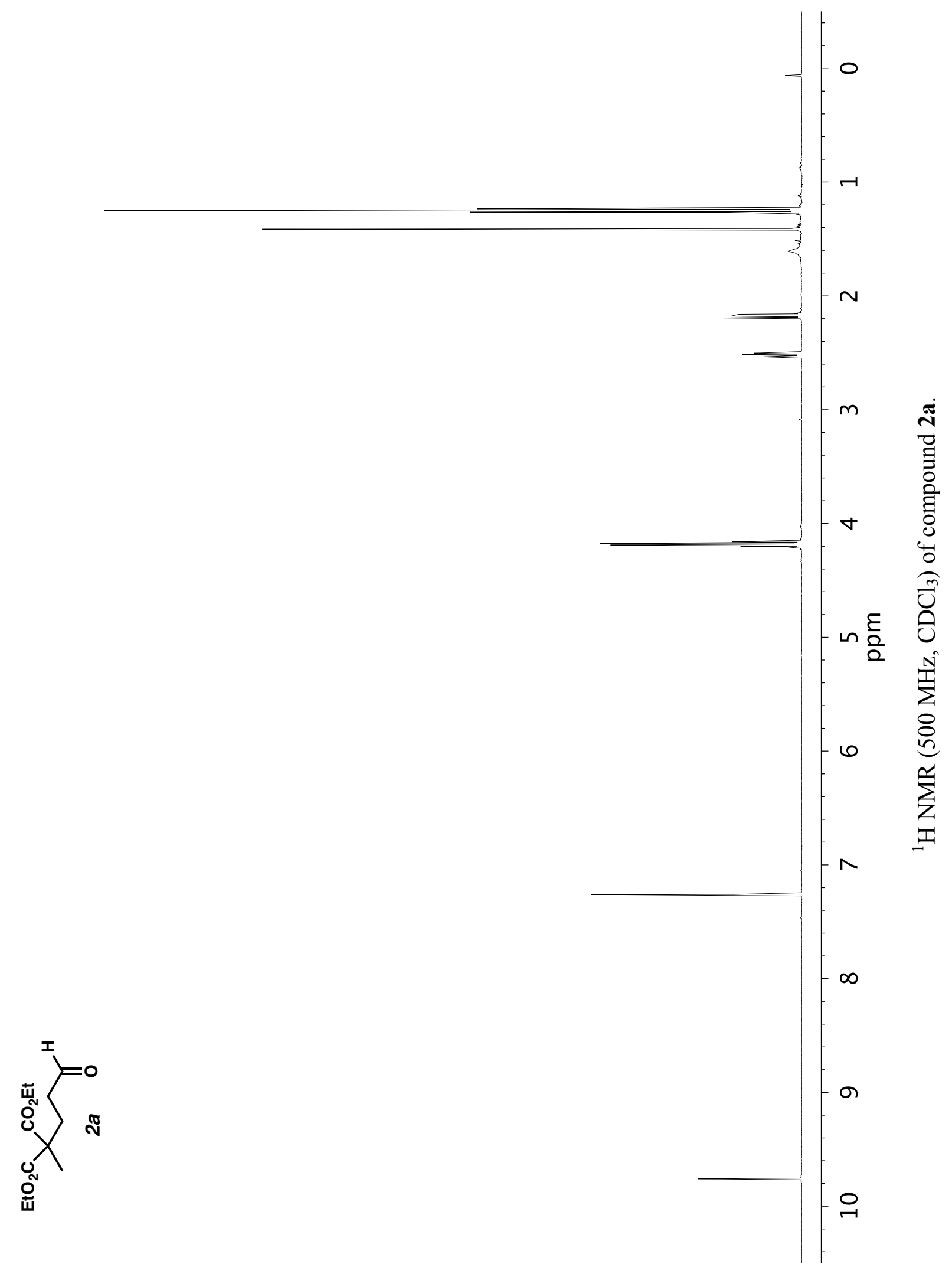



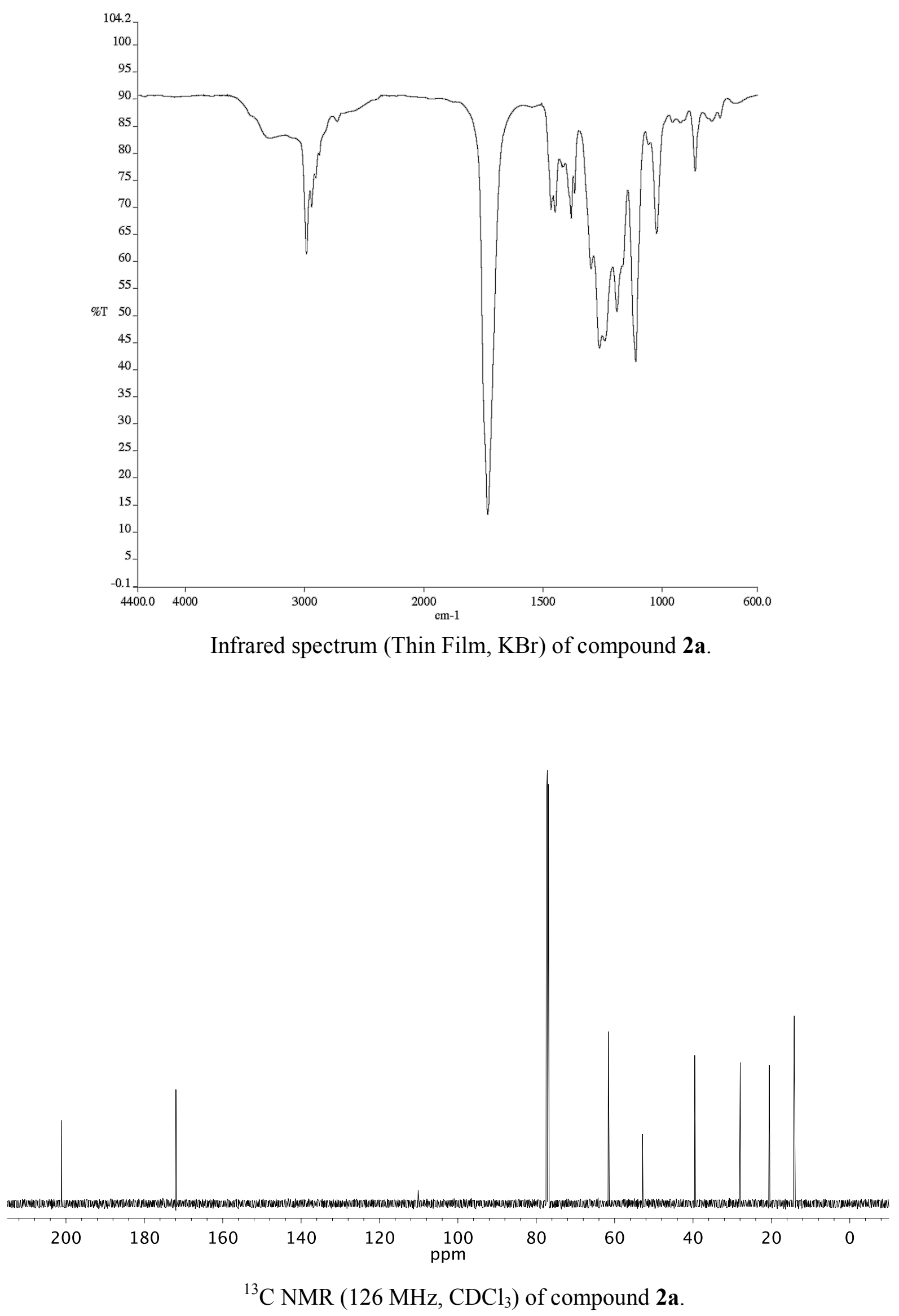


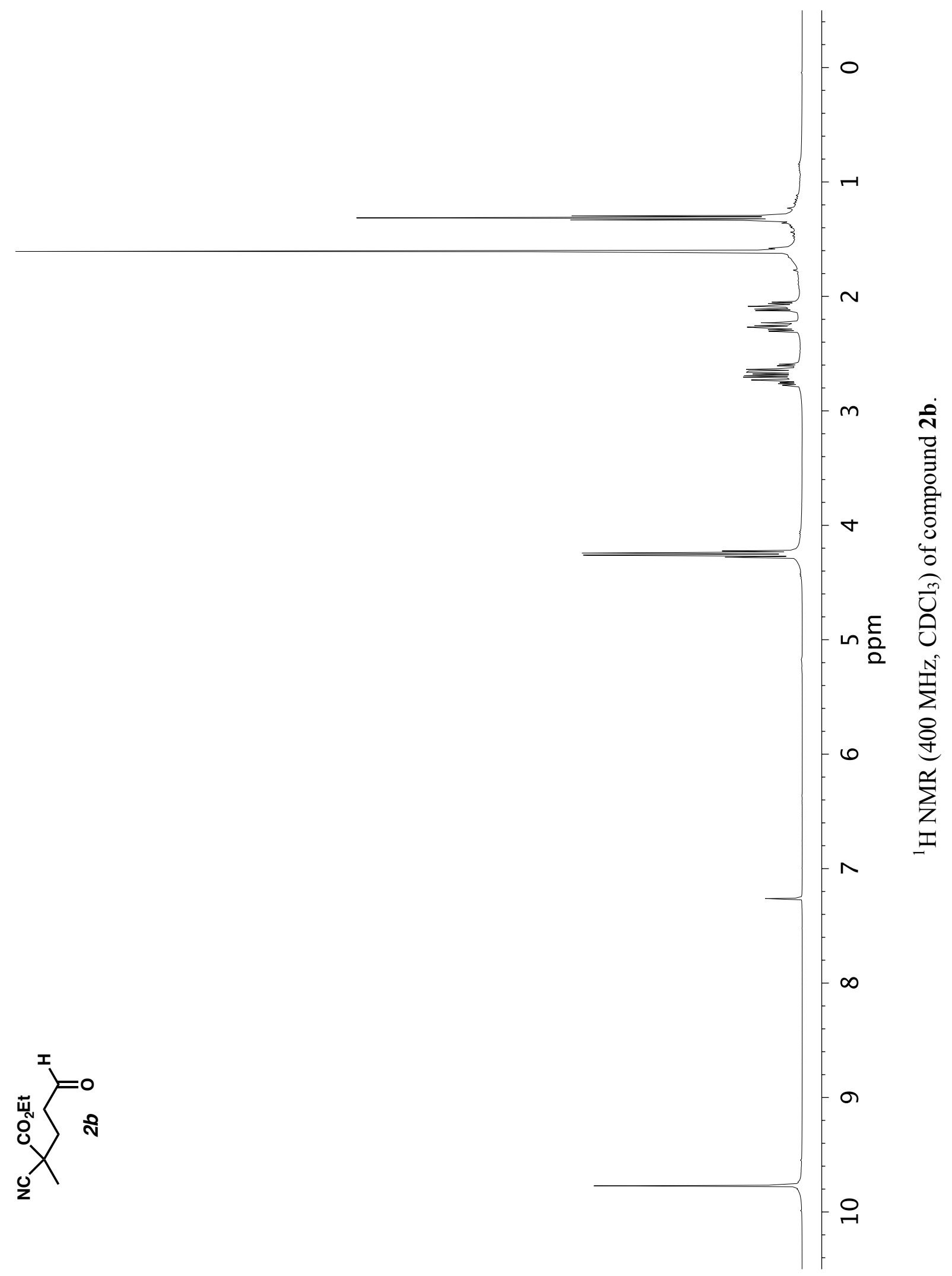




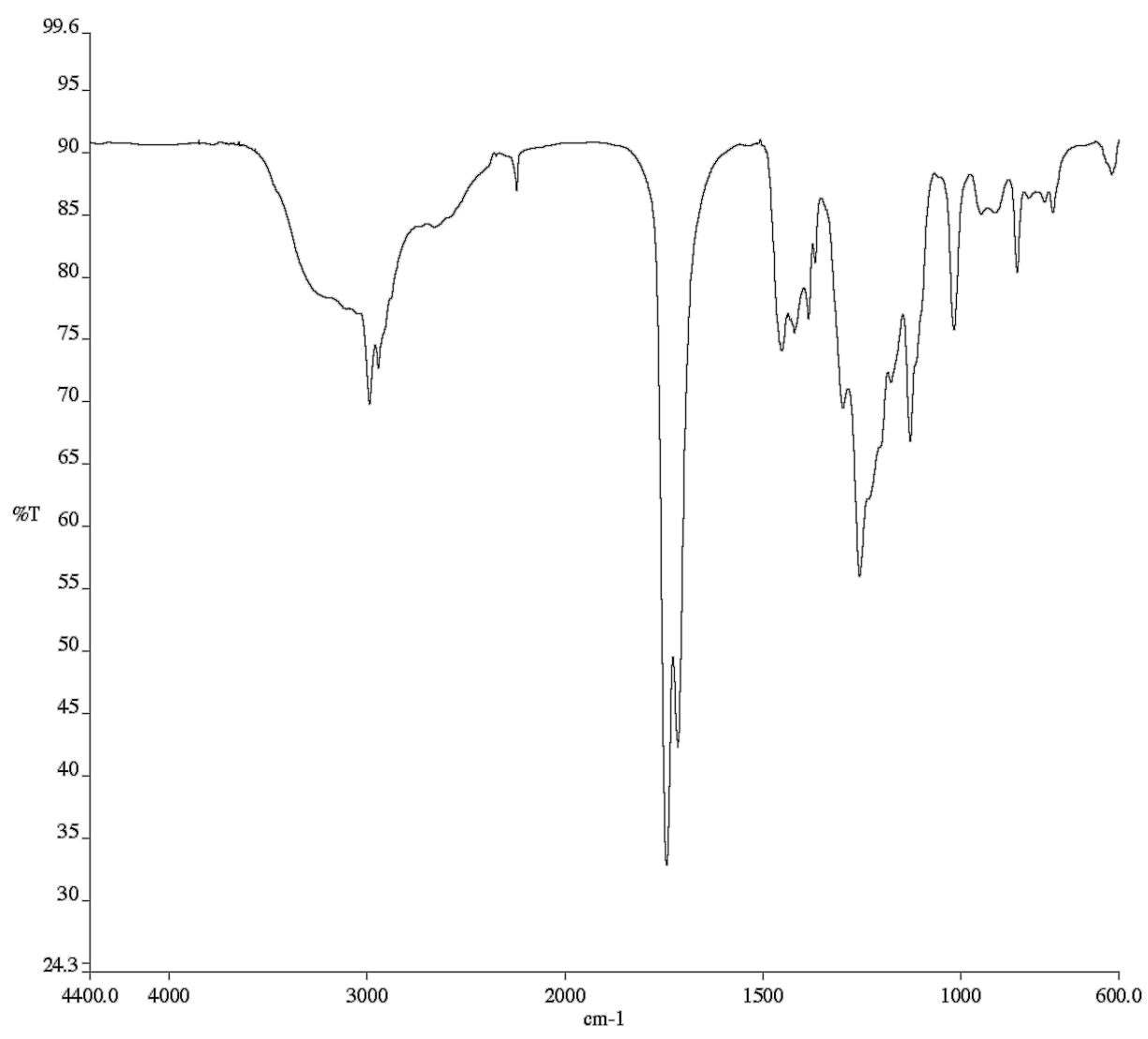

Infrared spectrum (Thin Film, KBr) of compound $\mathbf{2 b}$.

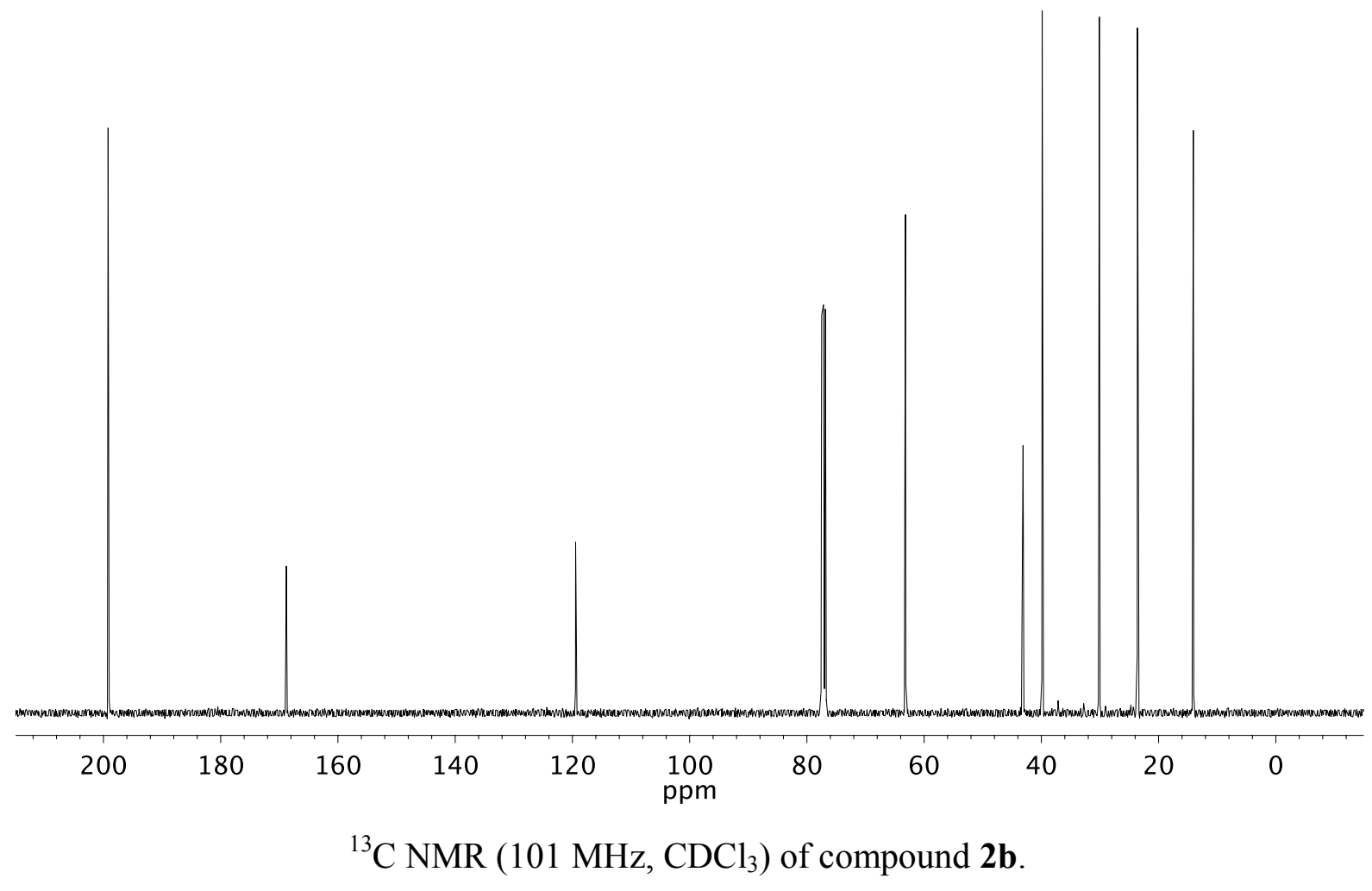




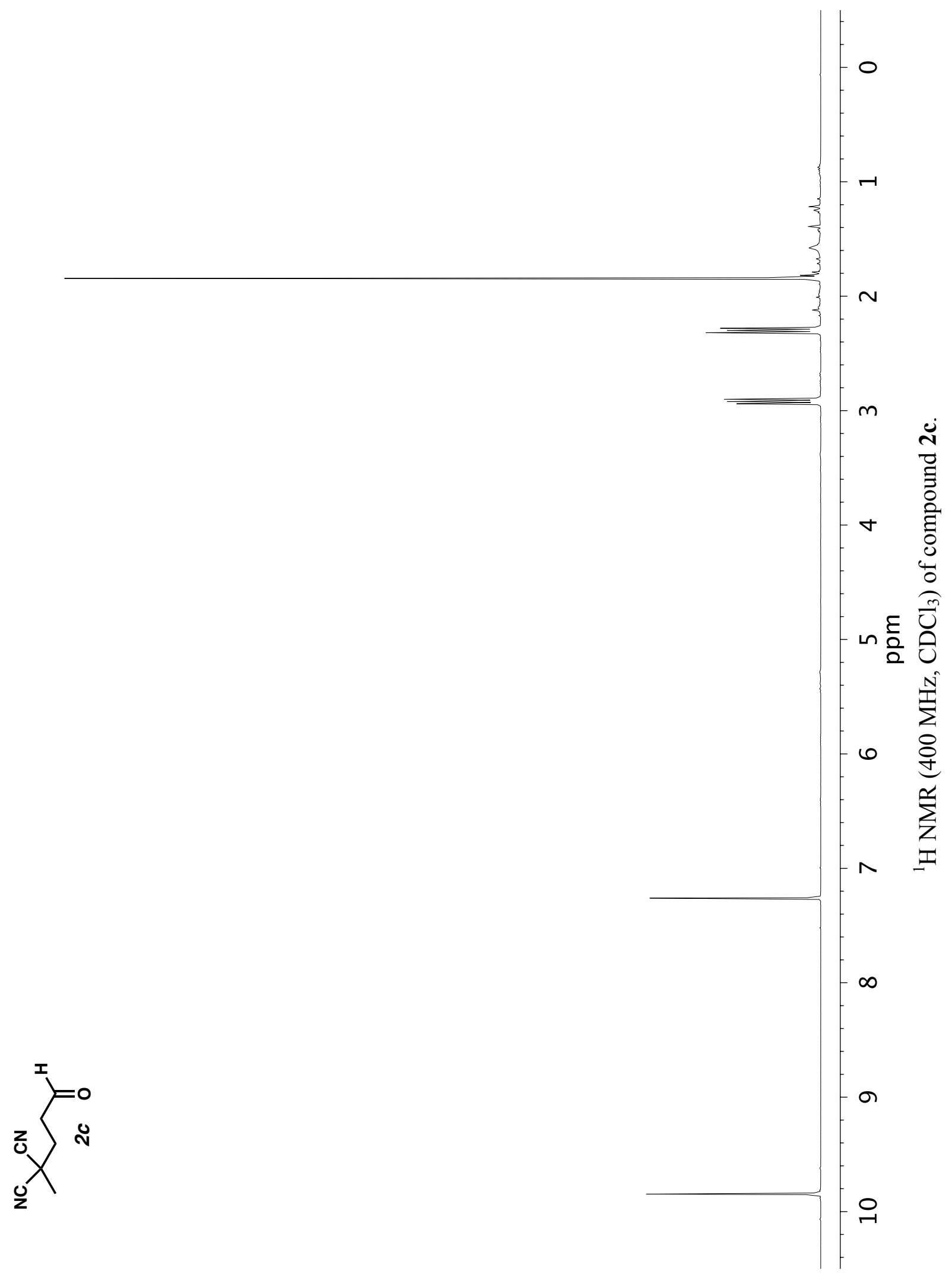




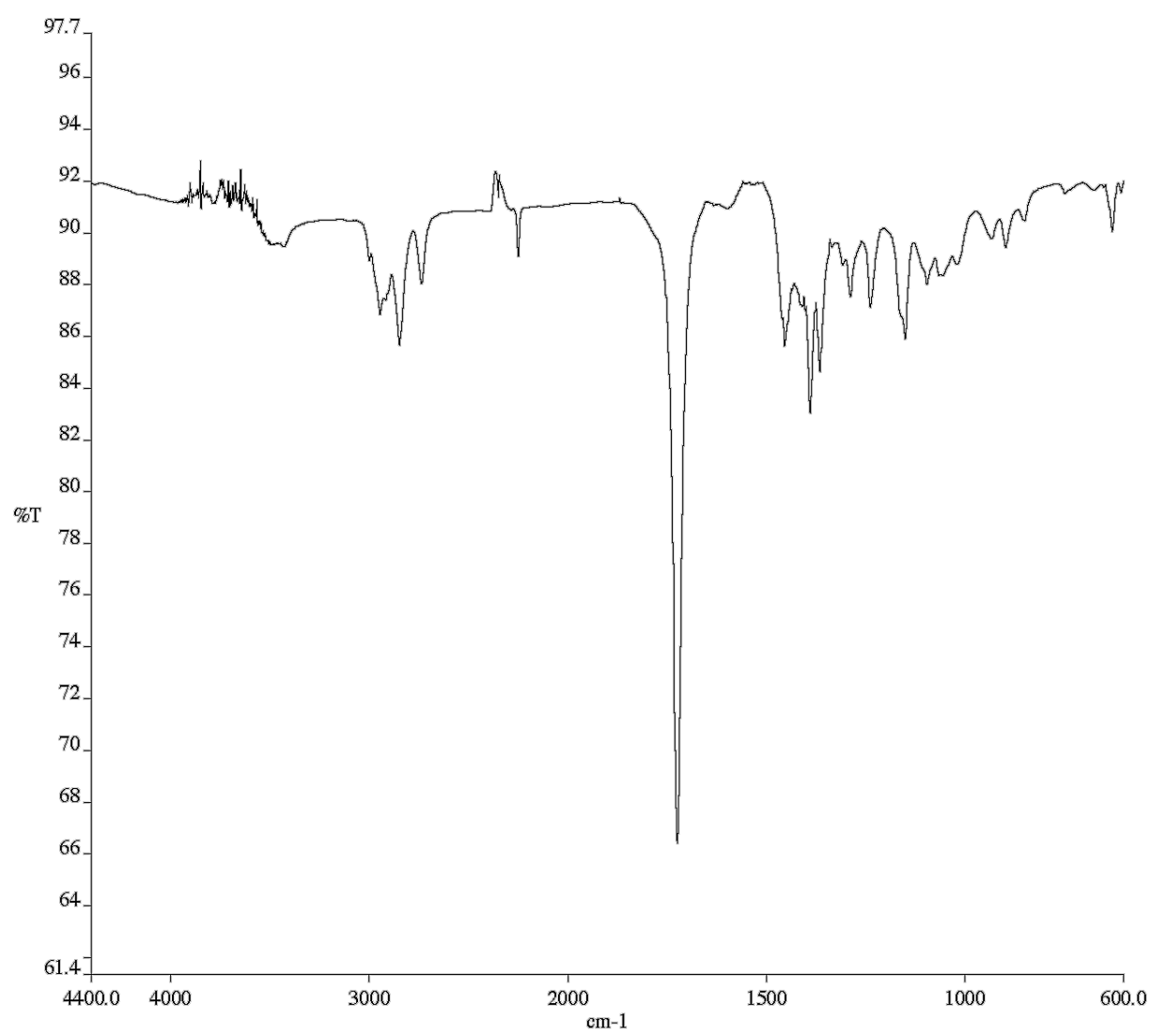

Infrared spectrum (Thin Film, KBr) of compound 2c.

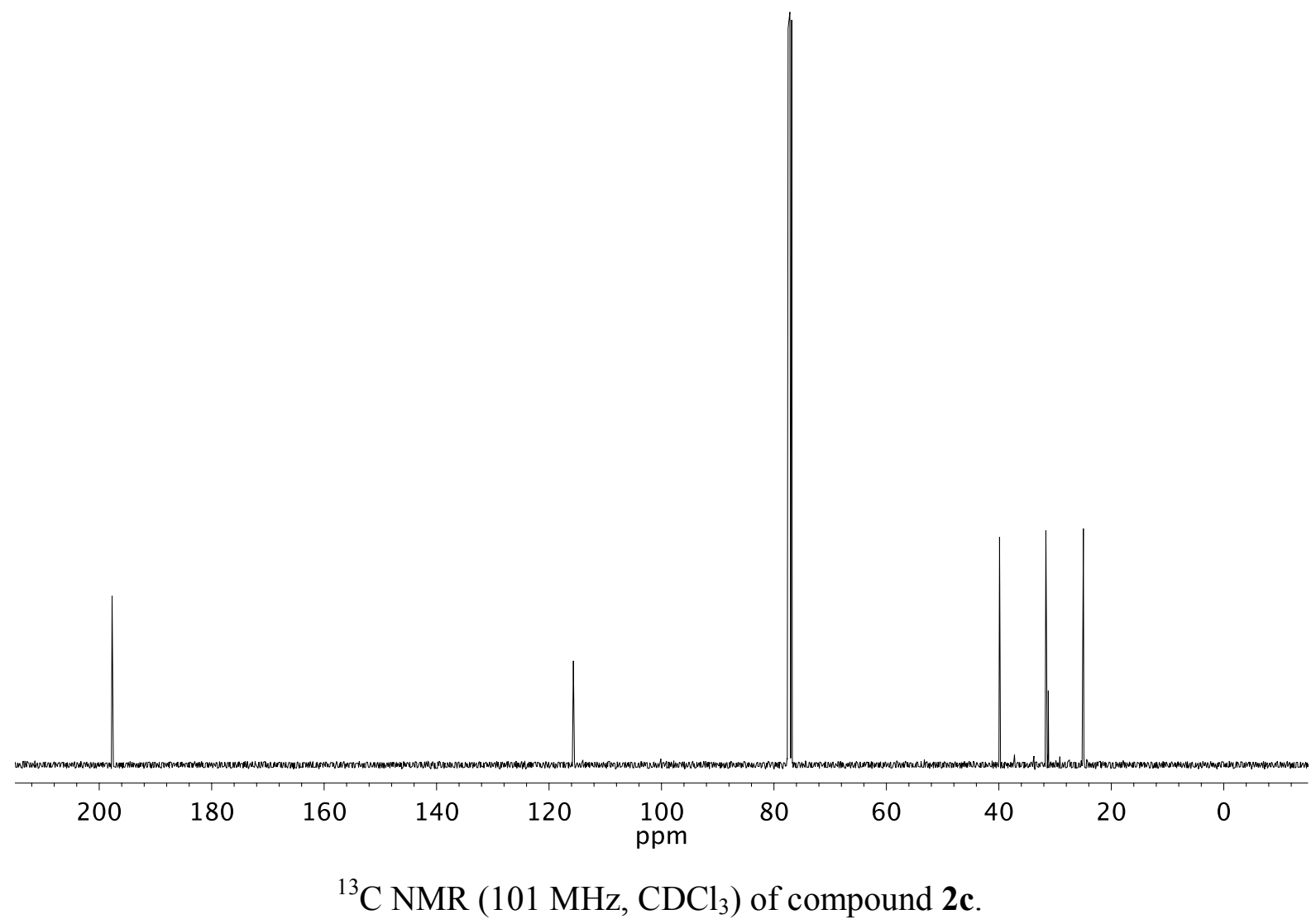




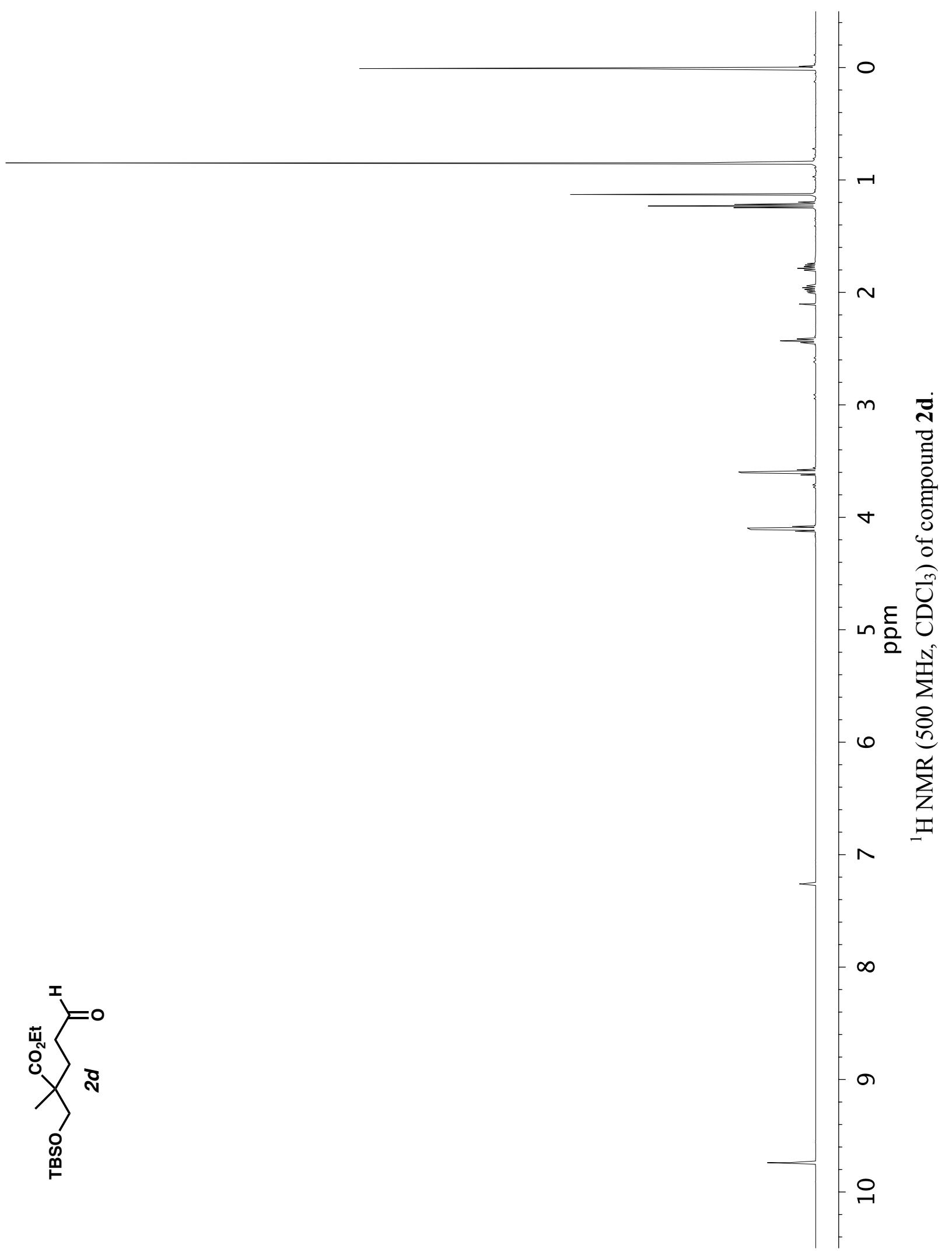



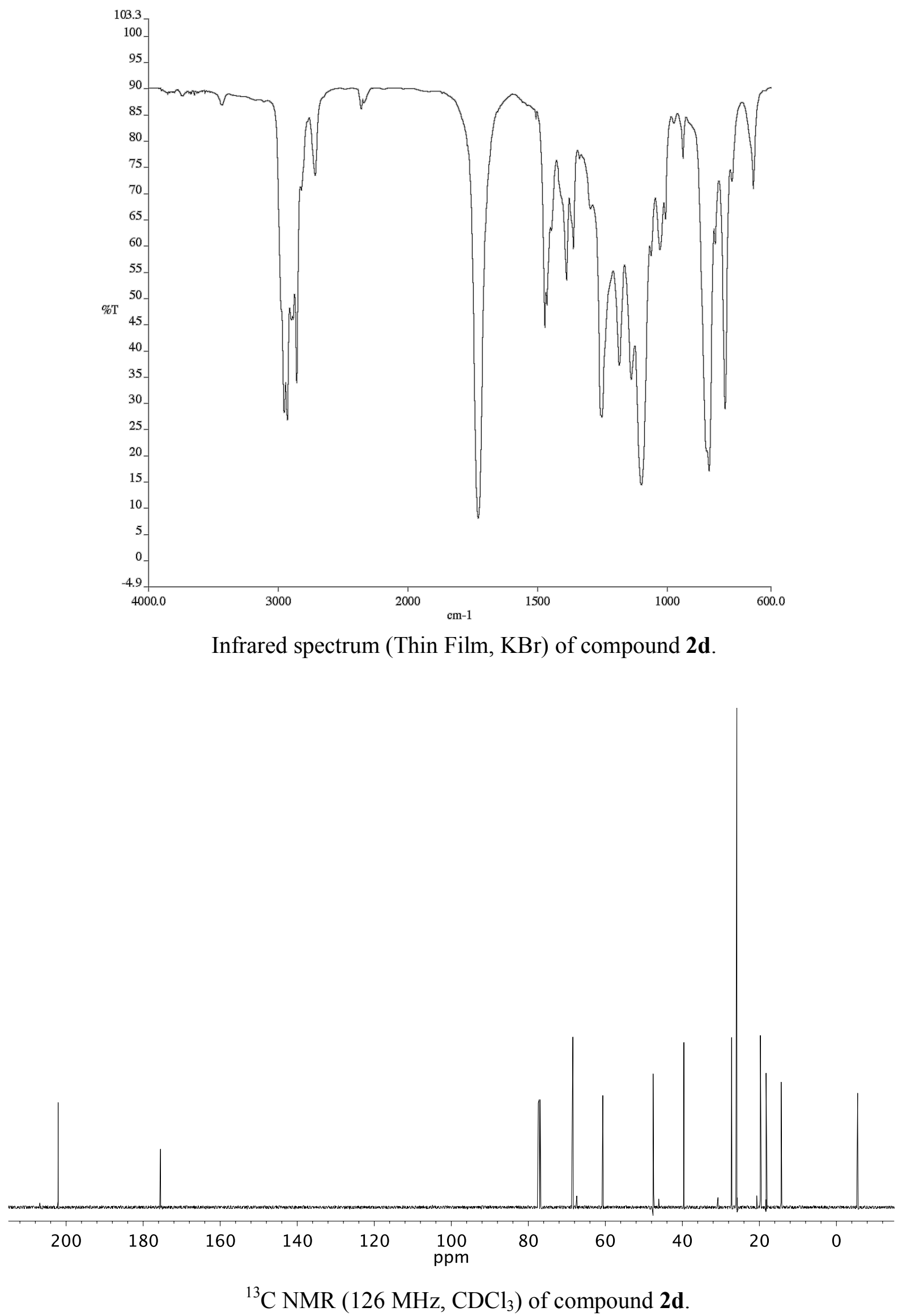


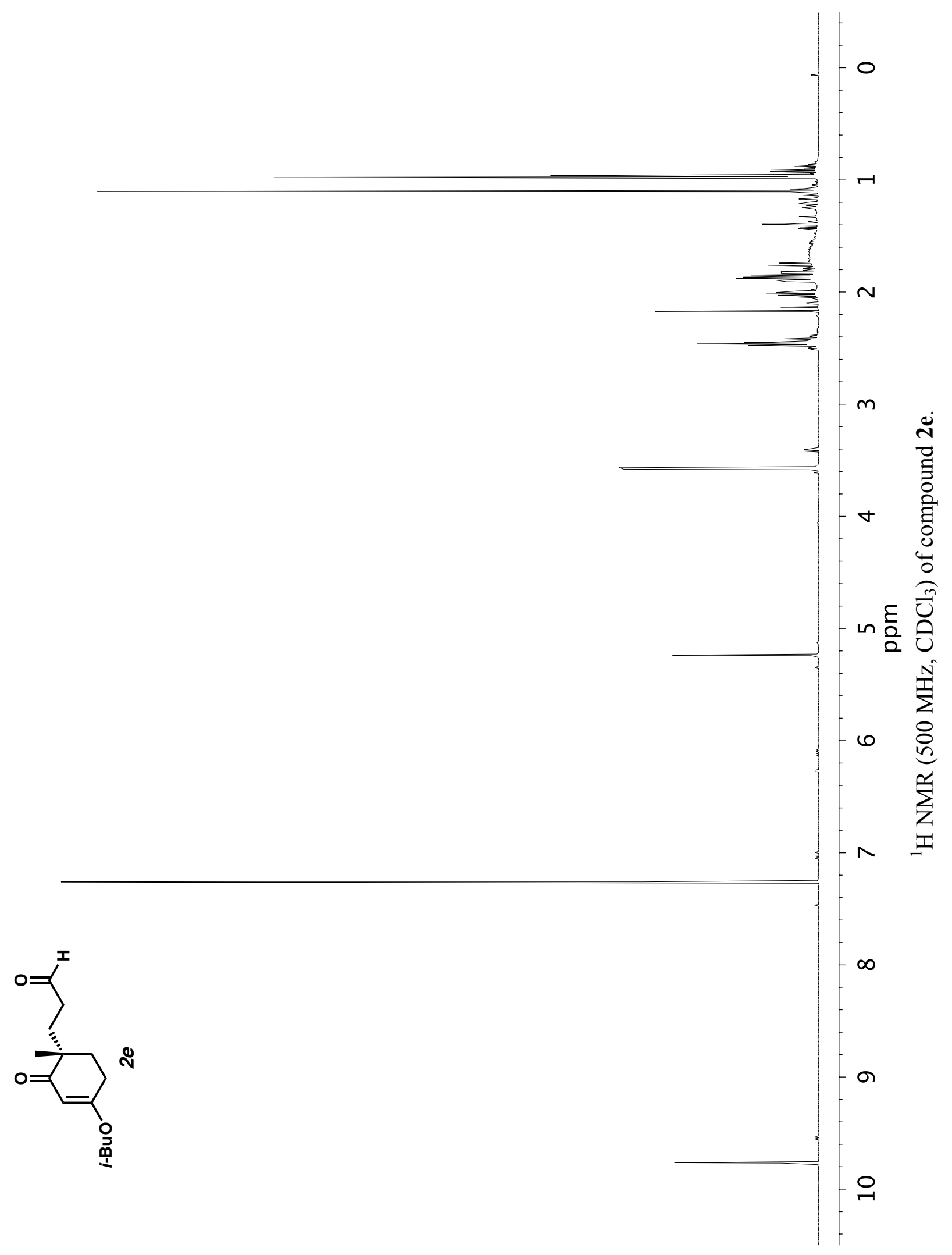



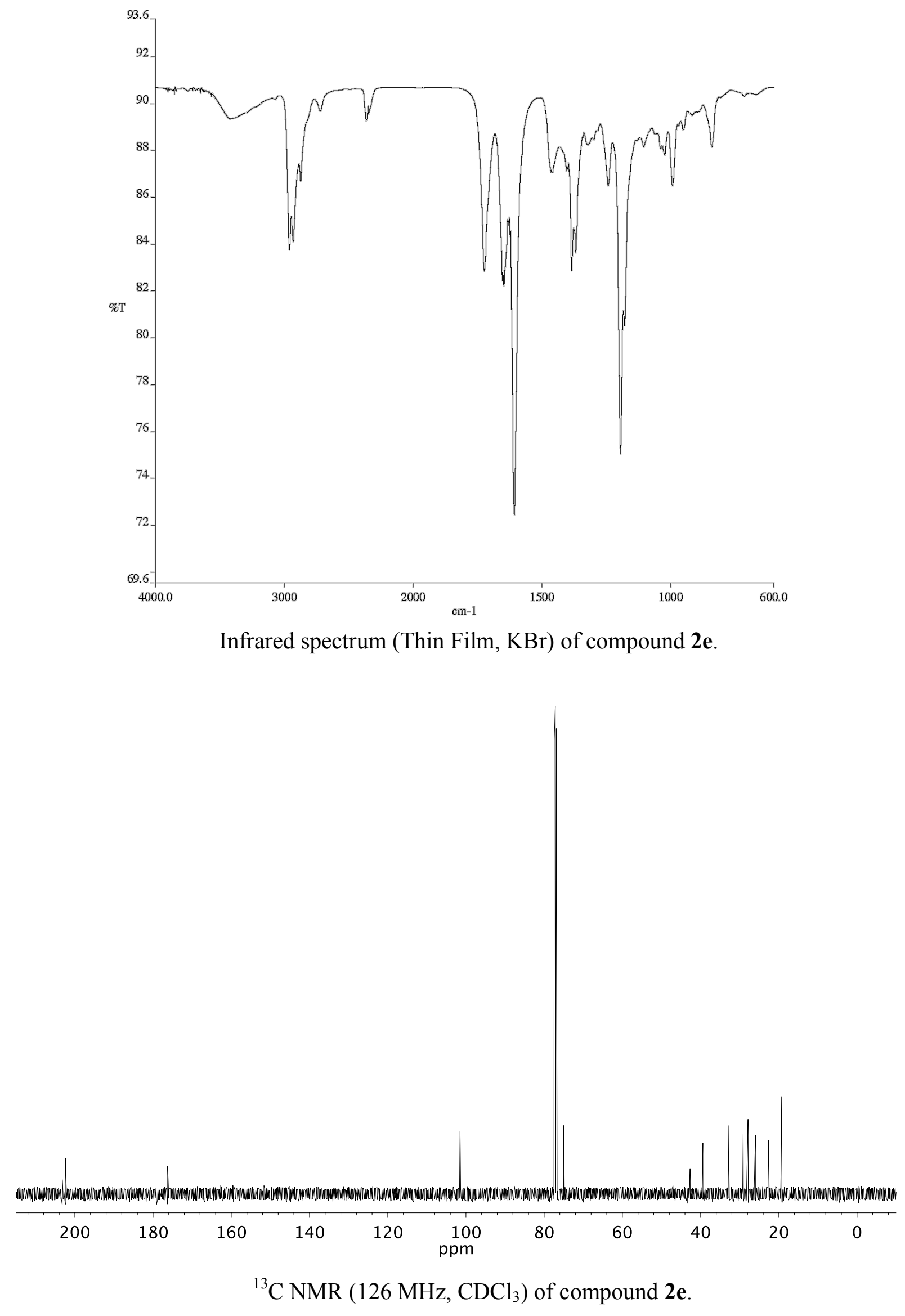


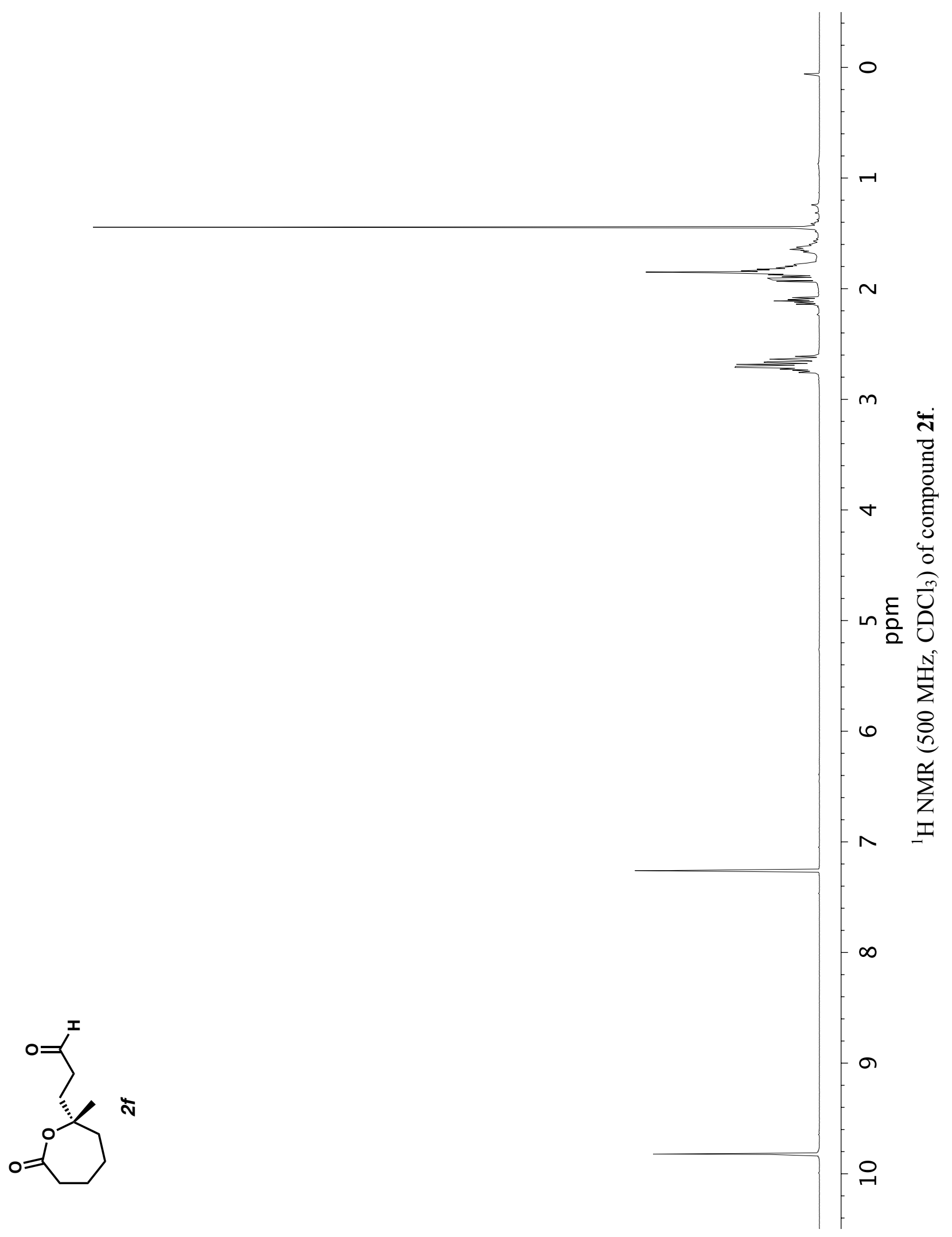



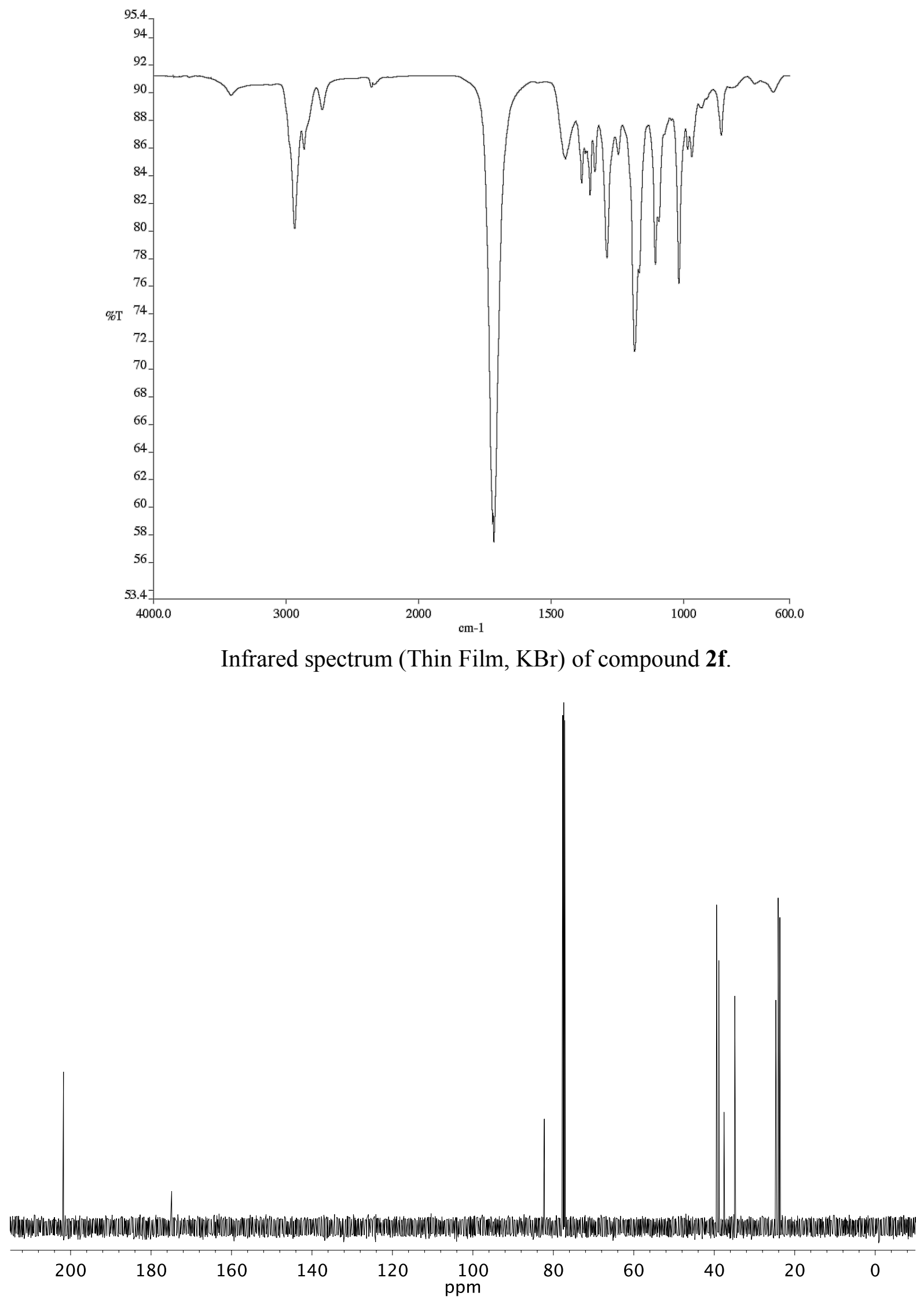

${ }^{13} \mathrm{C}$ NMR (126 MHz, $\mathrm{CDCl}_{3}$ ) of compound $\mathbf{2 f}$. 


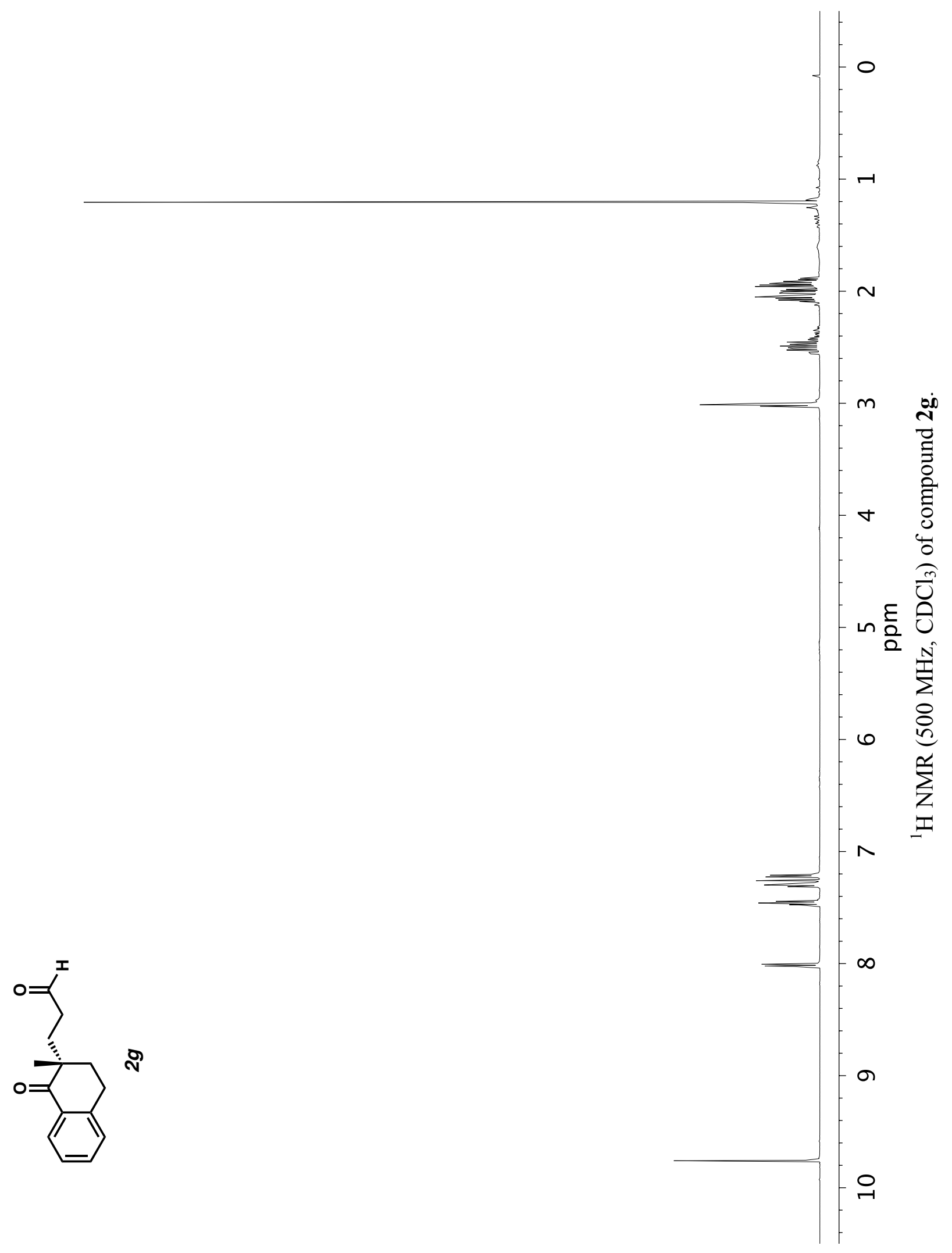




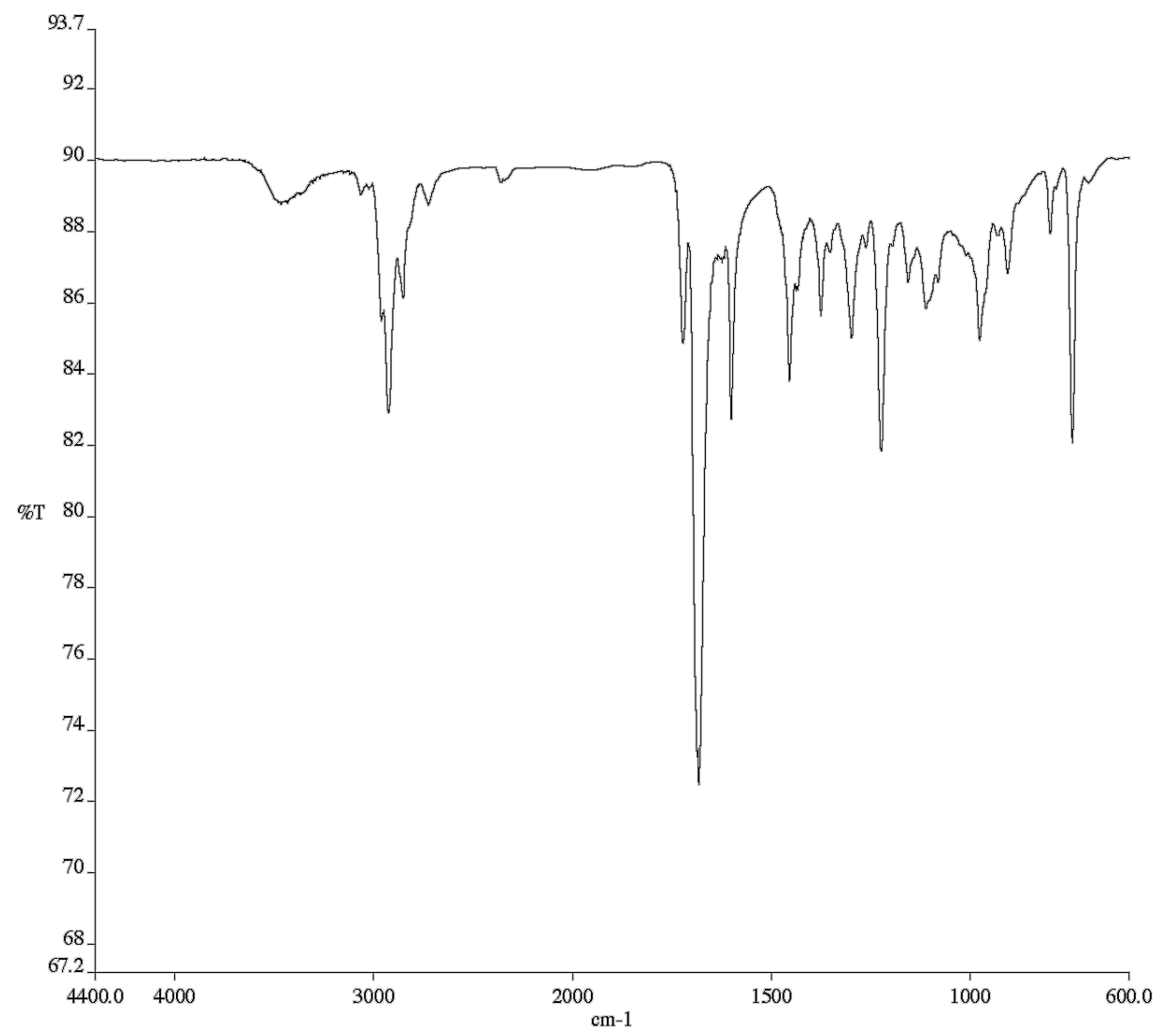

Infrared spectrum (Thin Film, KBr) of compound $\mathbf{2 g}$.

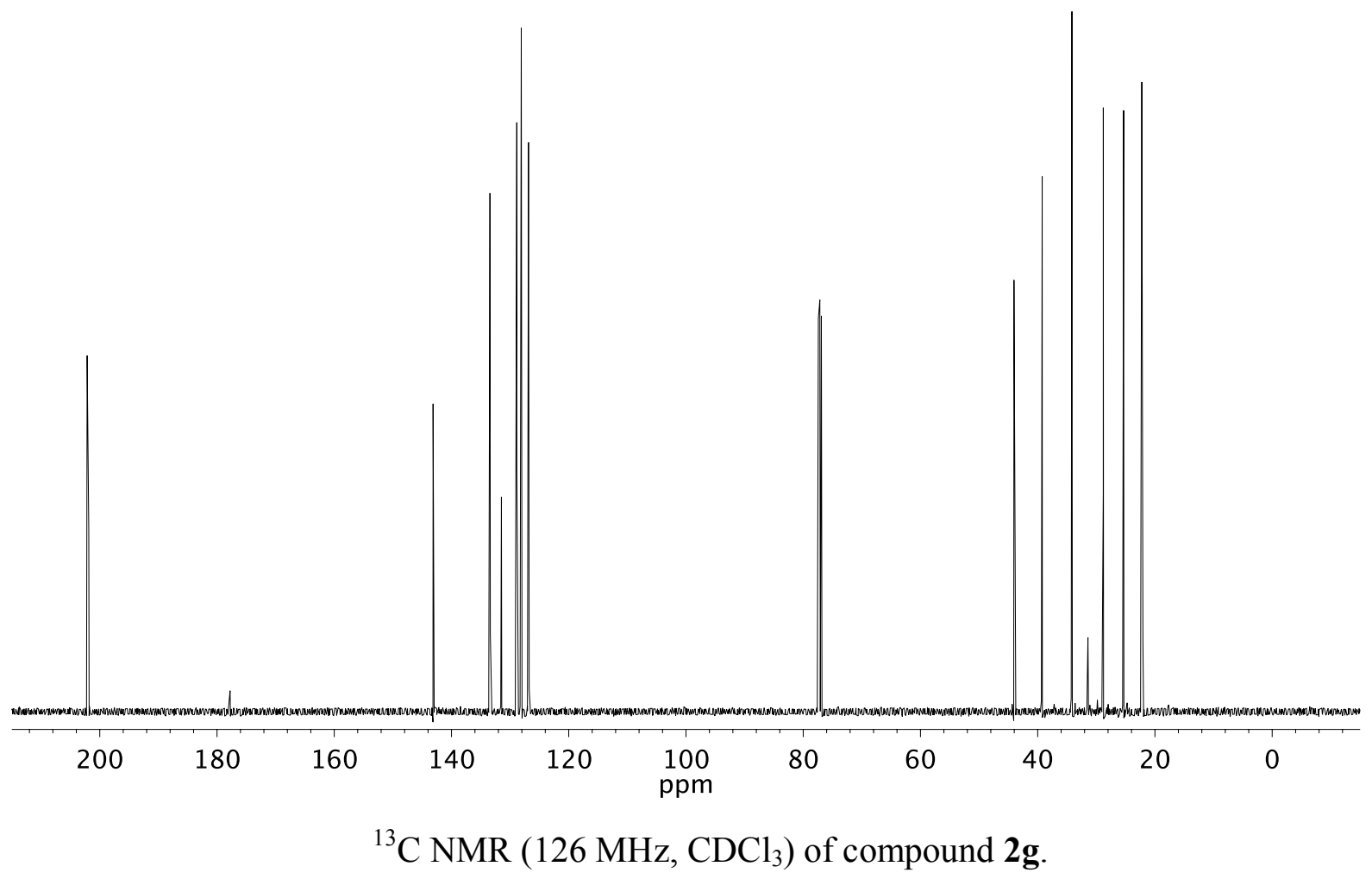




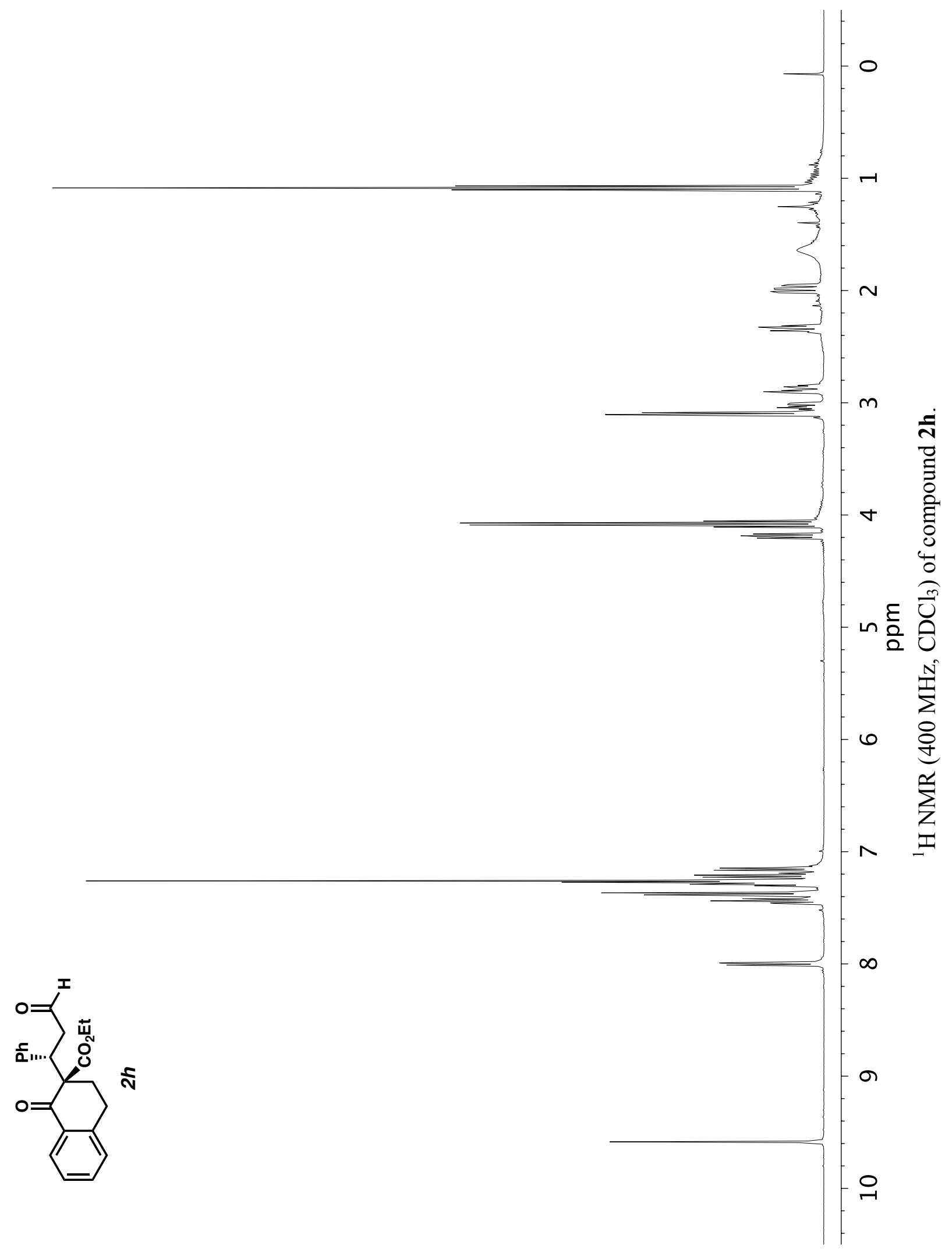




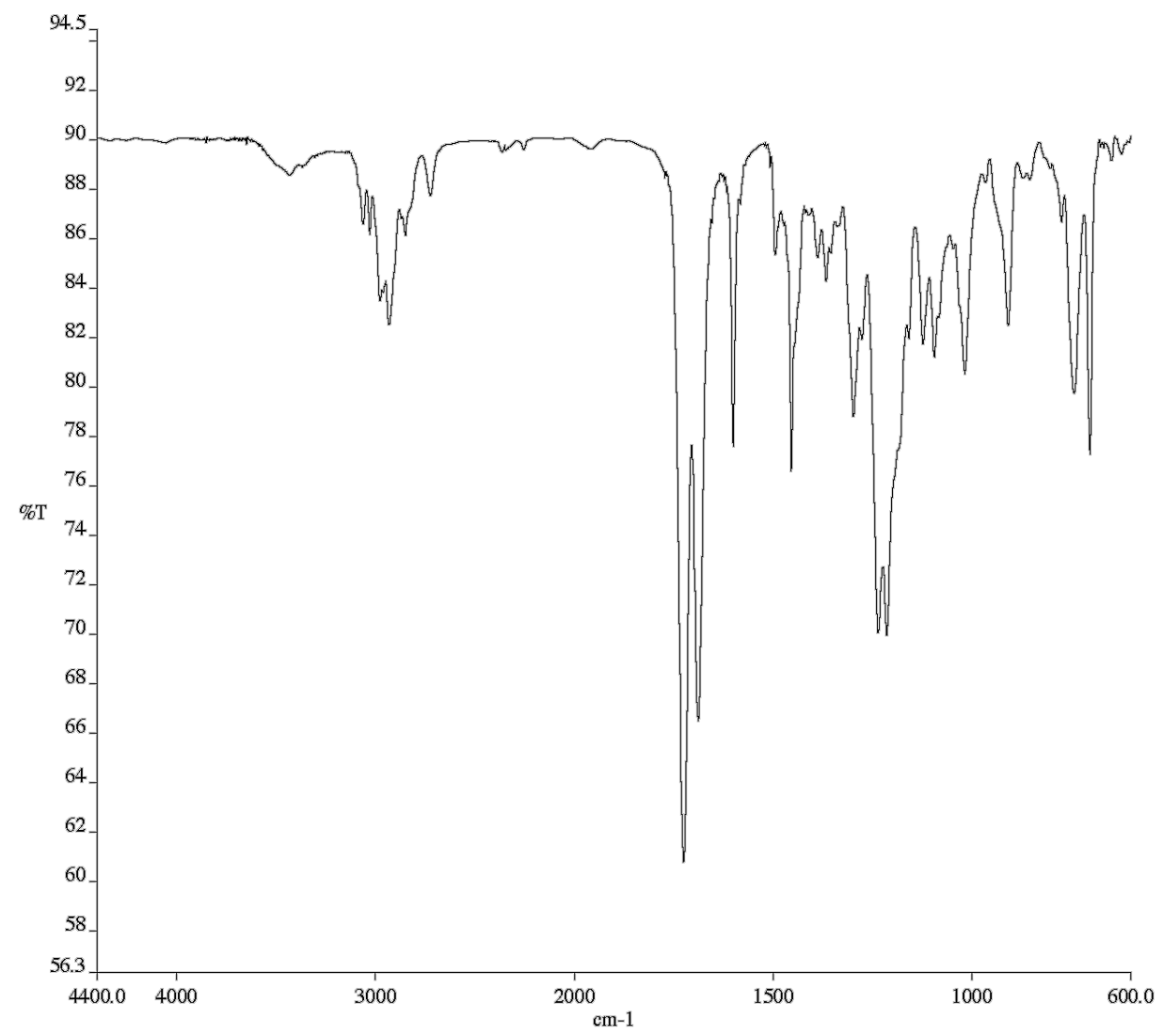

Infrared spectrum (Thin Film, KBr) of compound $\mathbf{2 h}$.

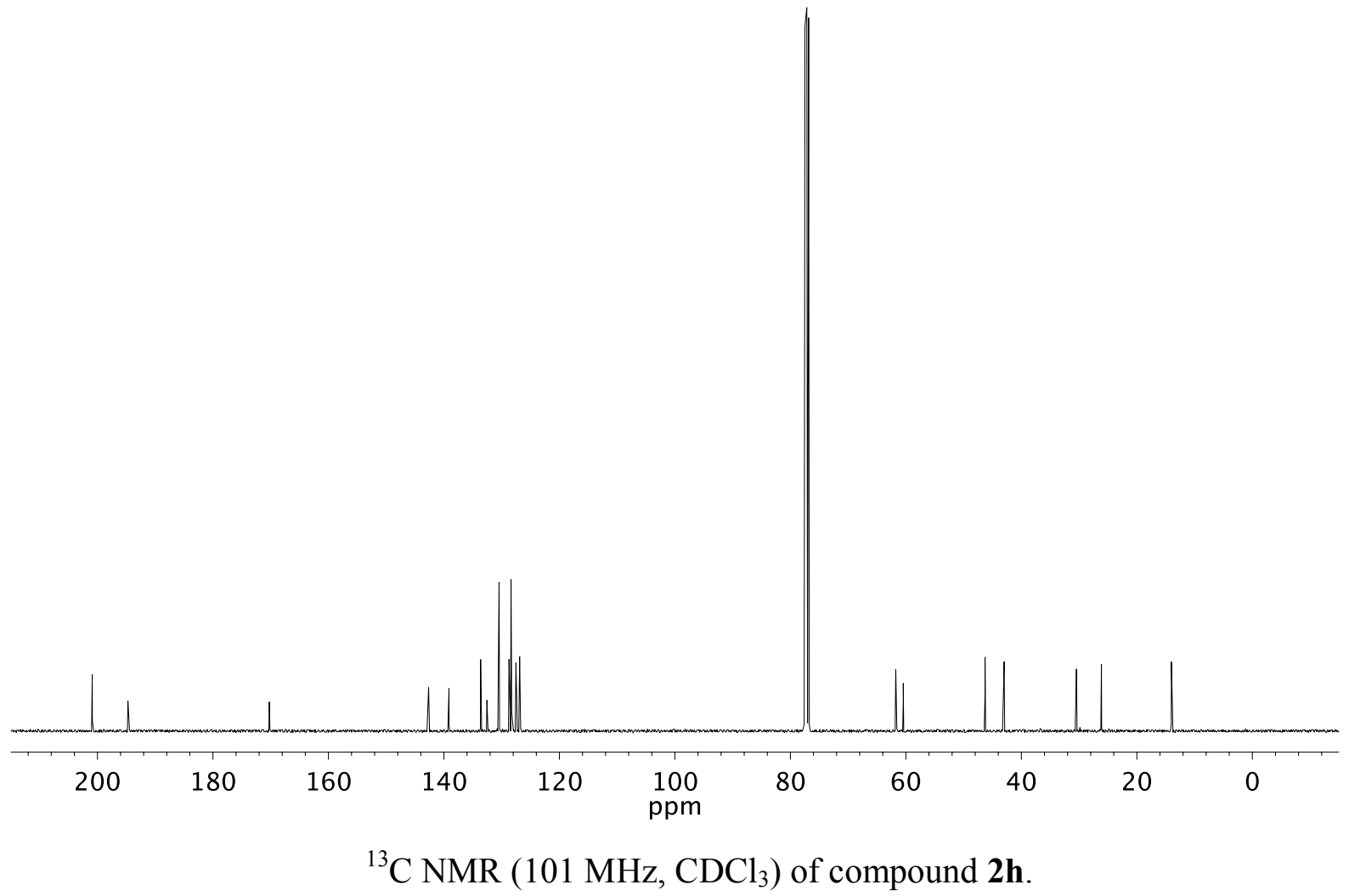




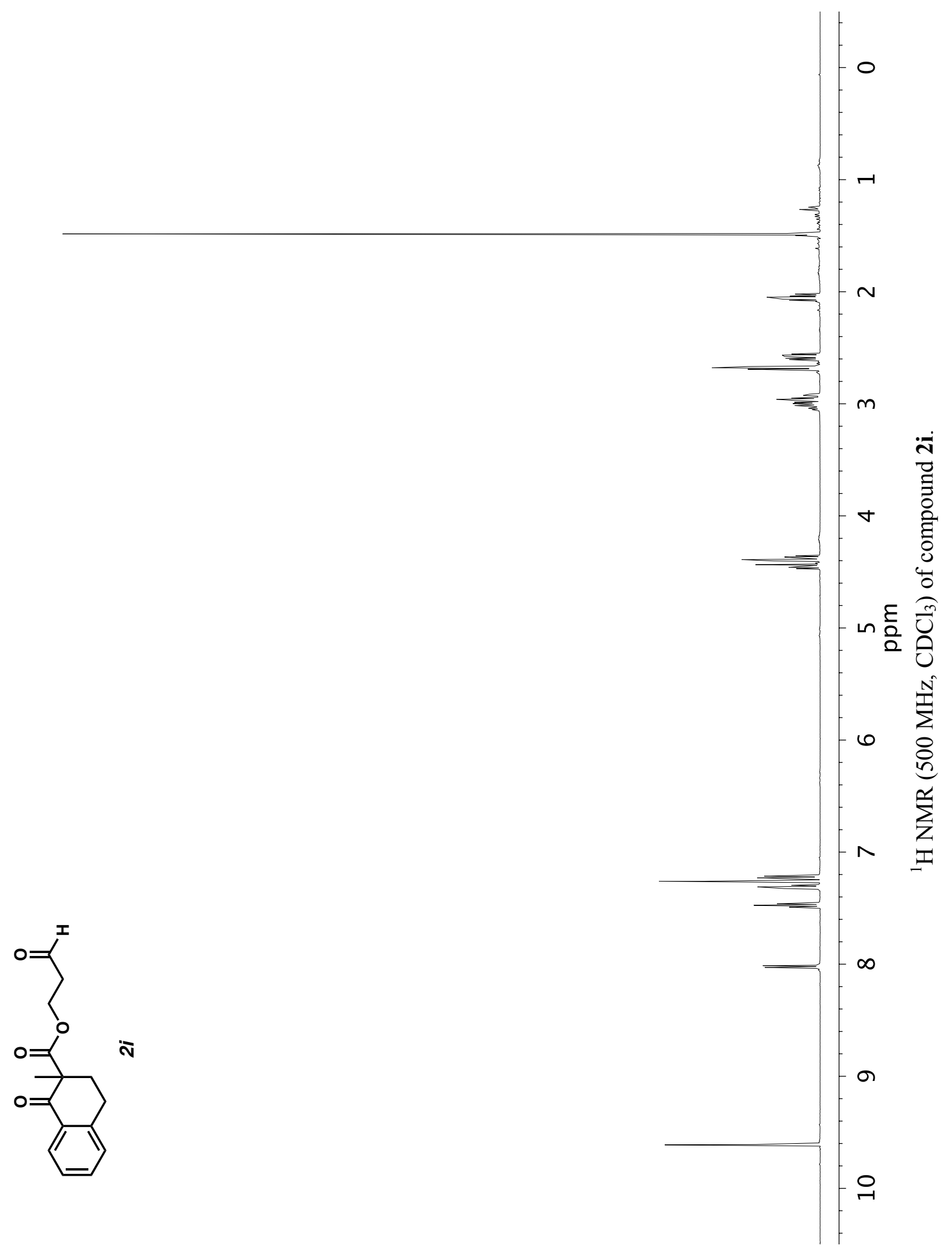



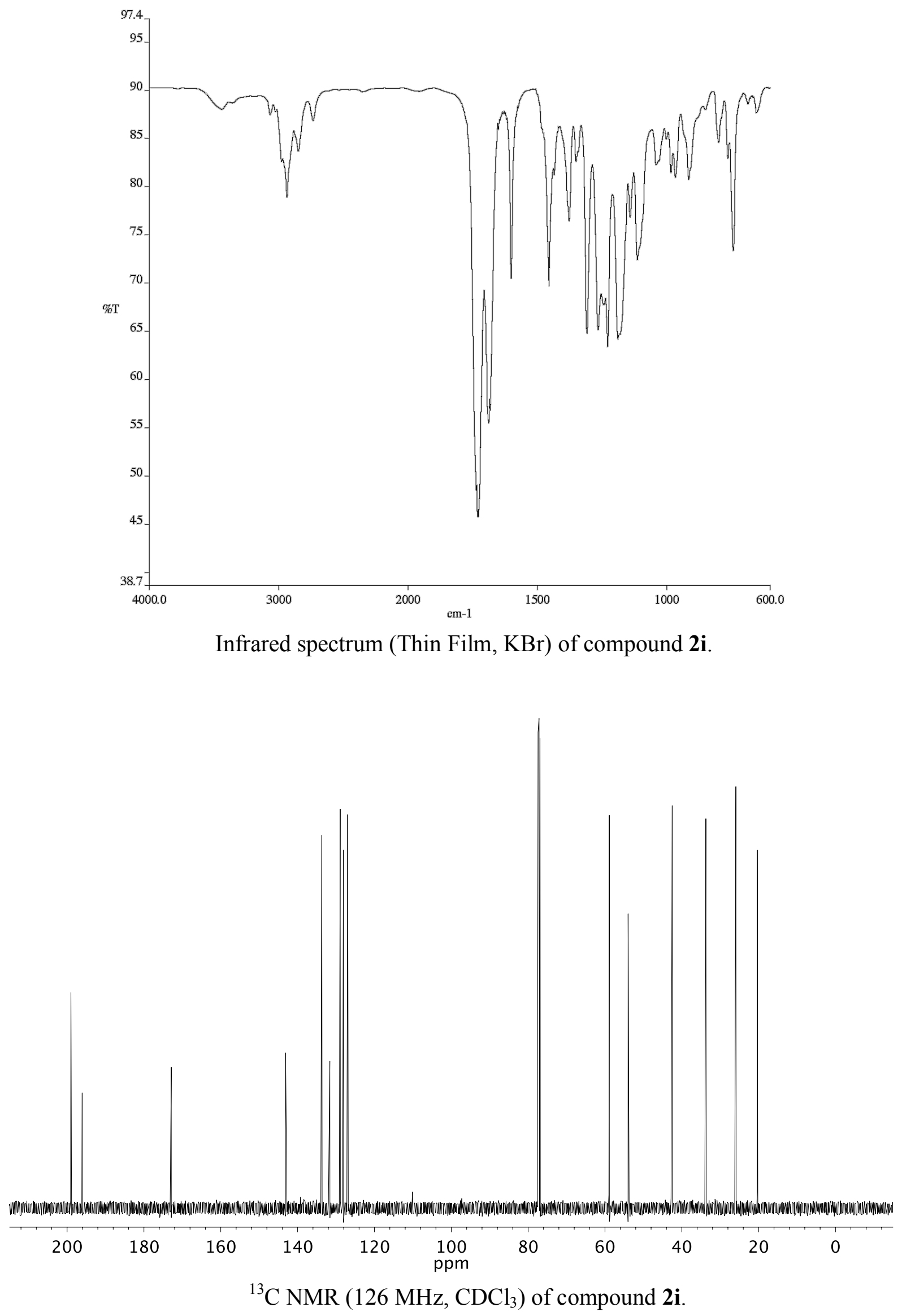


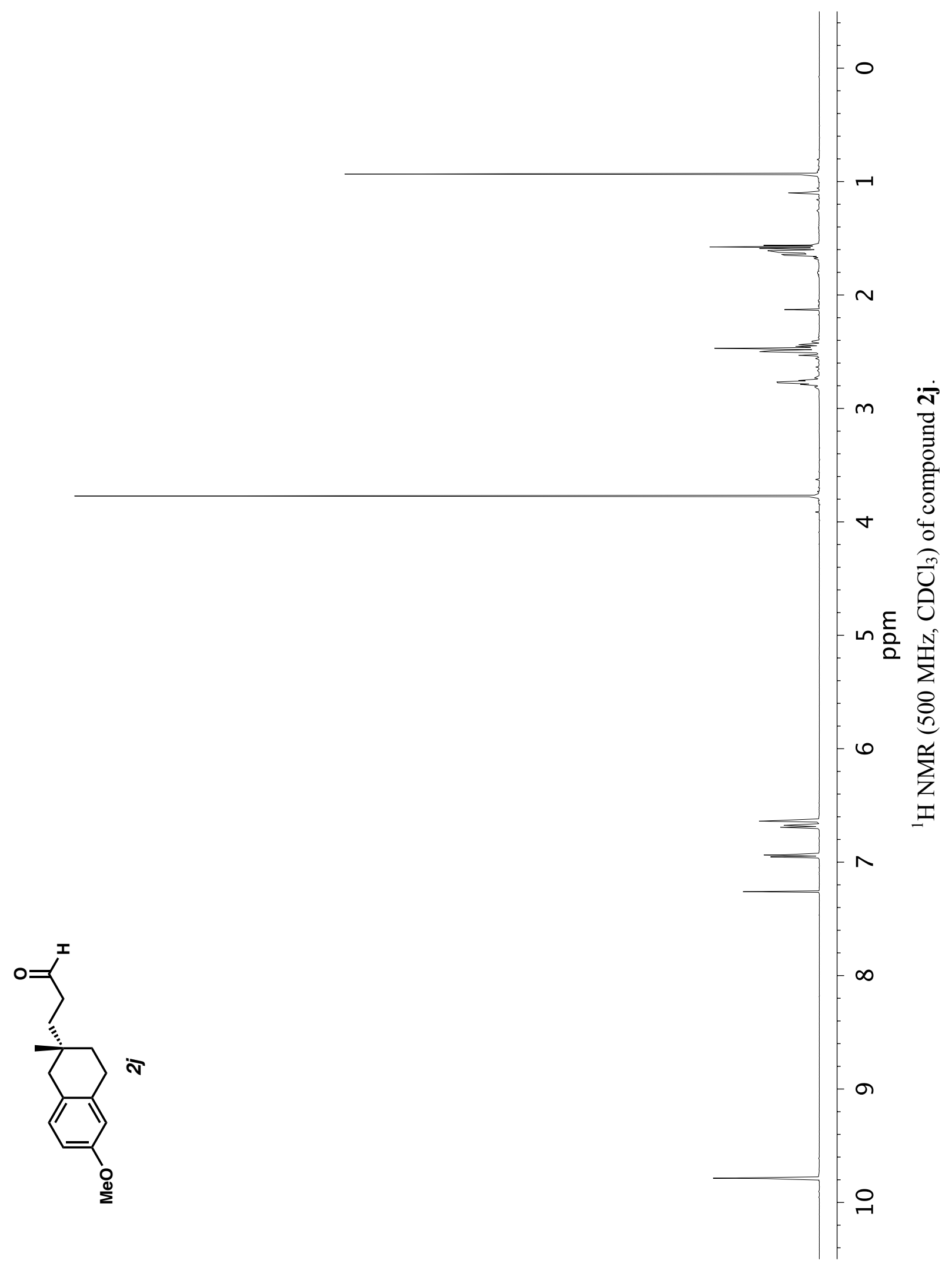



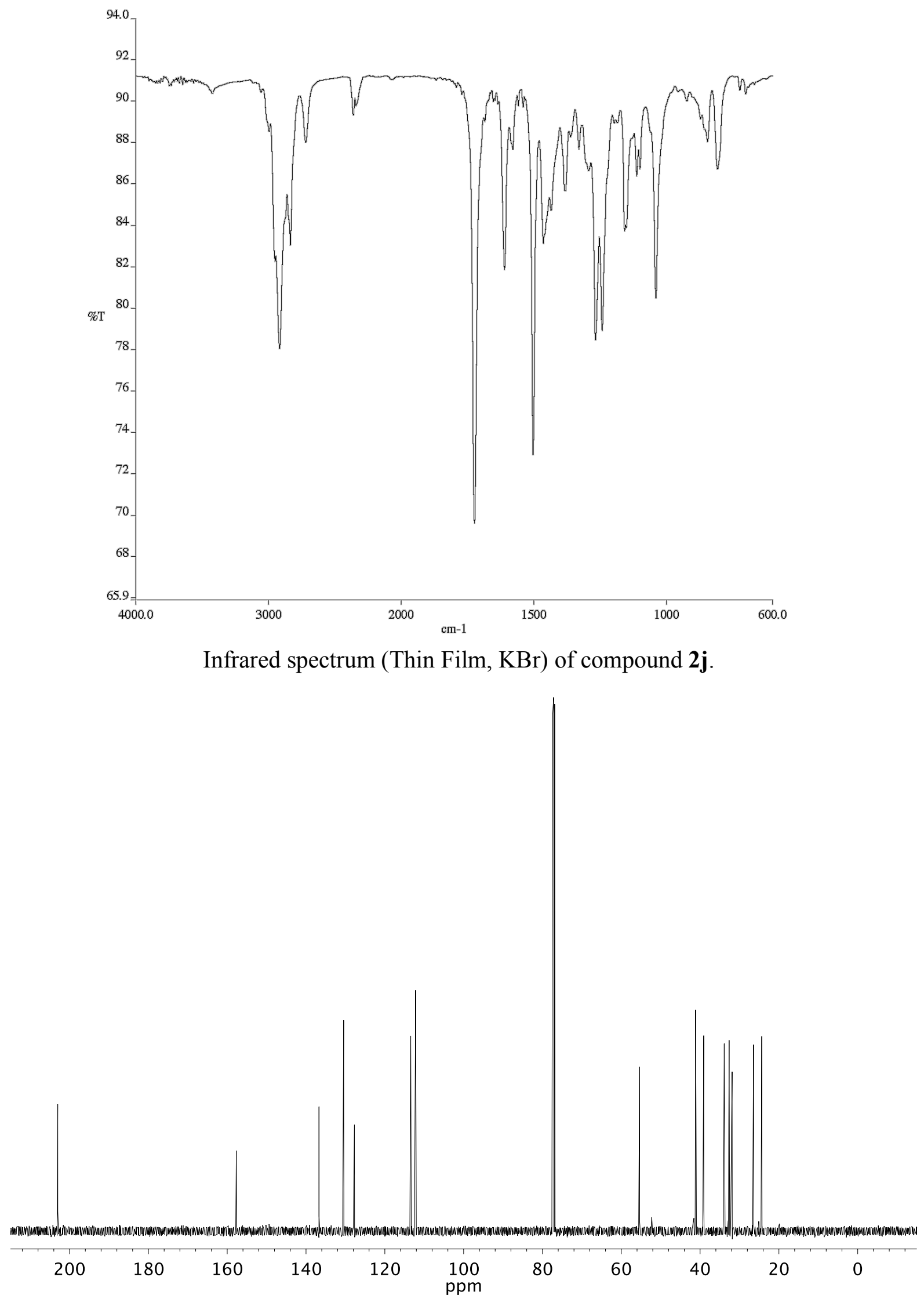

${ }^{13} \mathrm{C}$ NMR (126 MHz, $\mathrm{CDCl}_{3}$ ) of compound $\mathbf{2 j}$. 


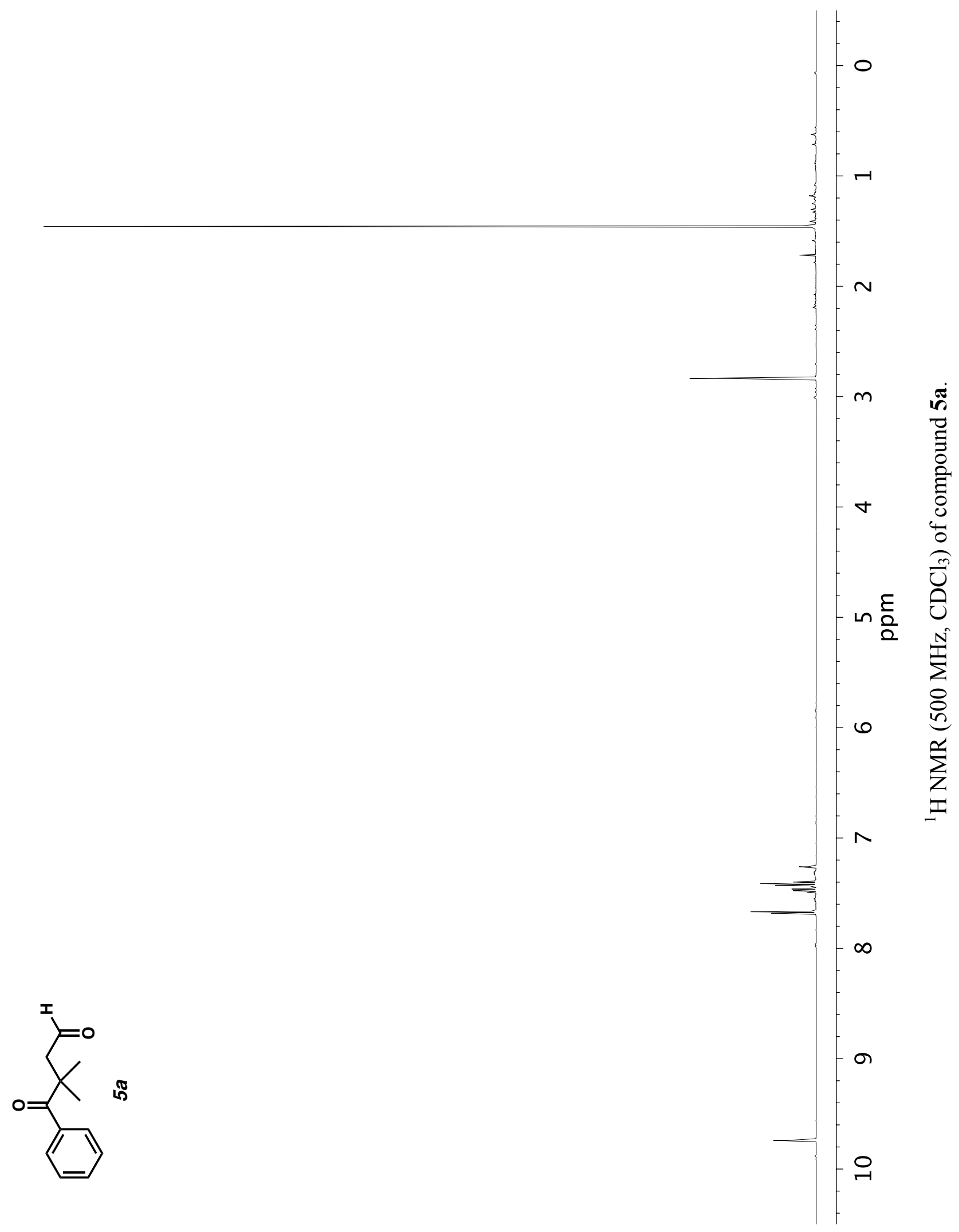




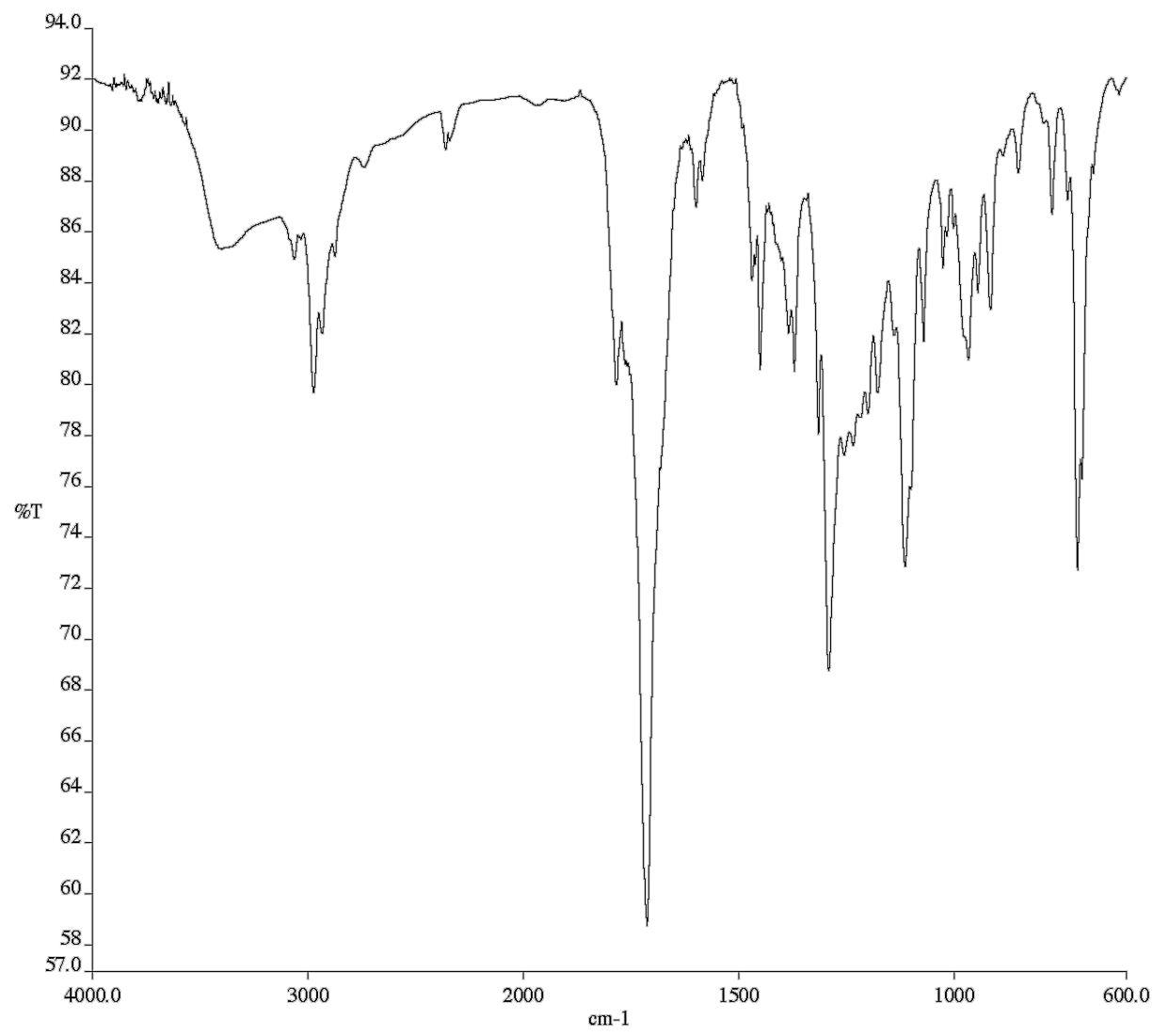

Infrared spectrum (Thin Film, KBr) of compound 5a.

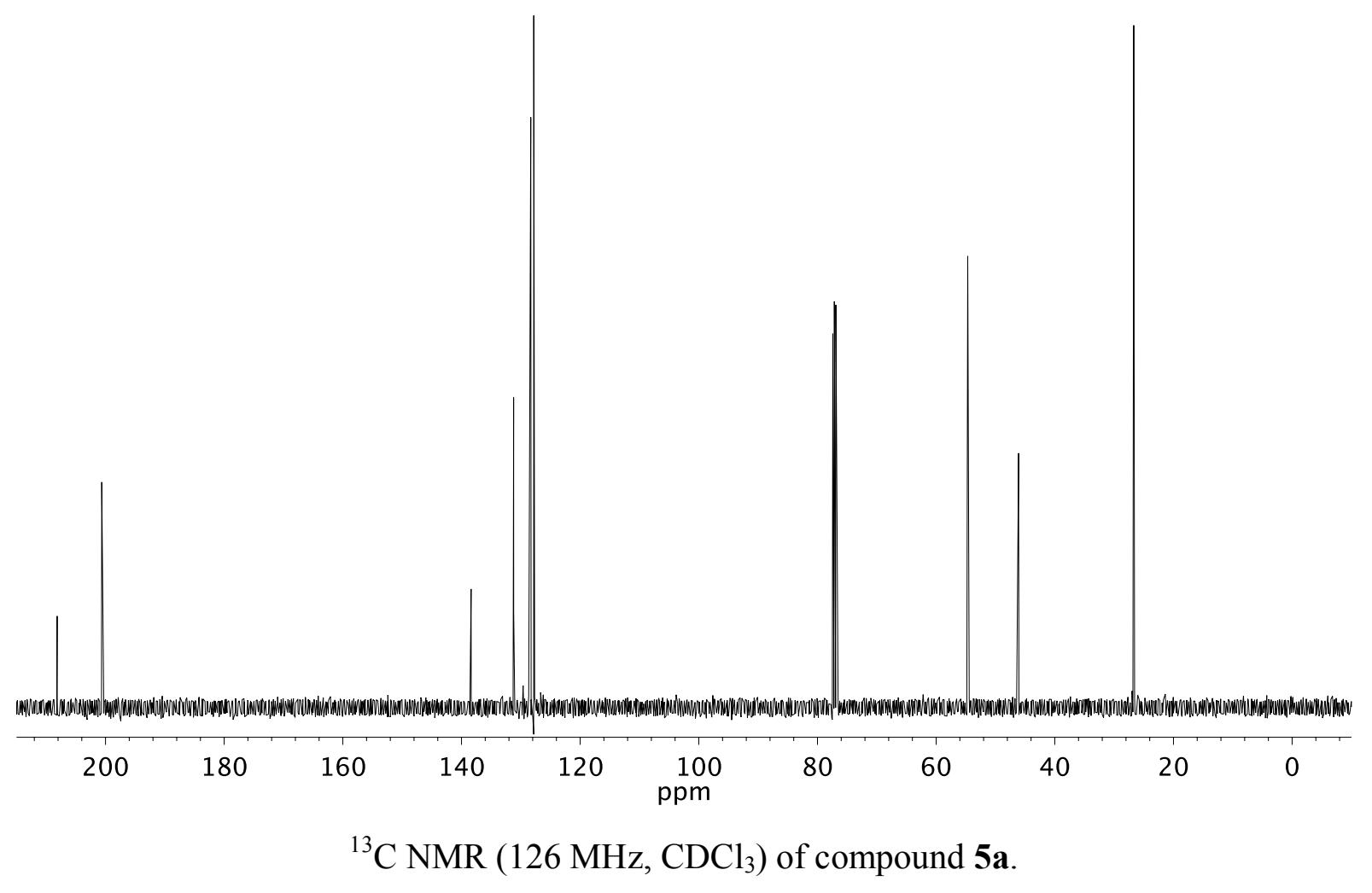




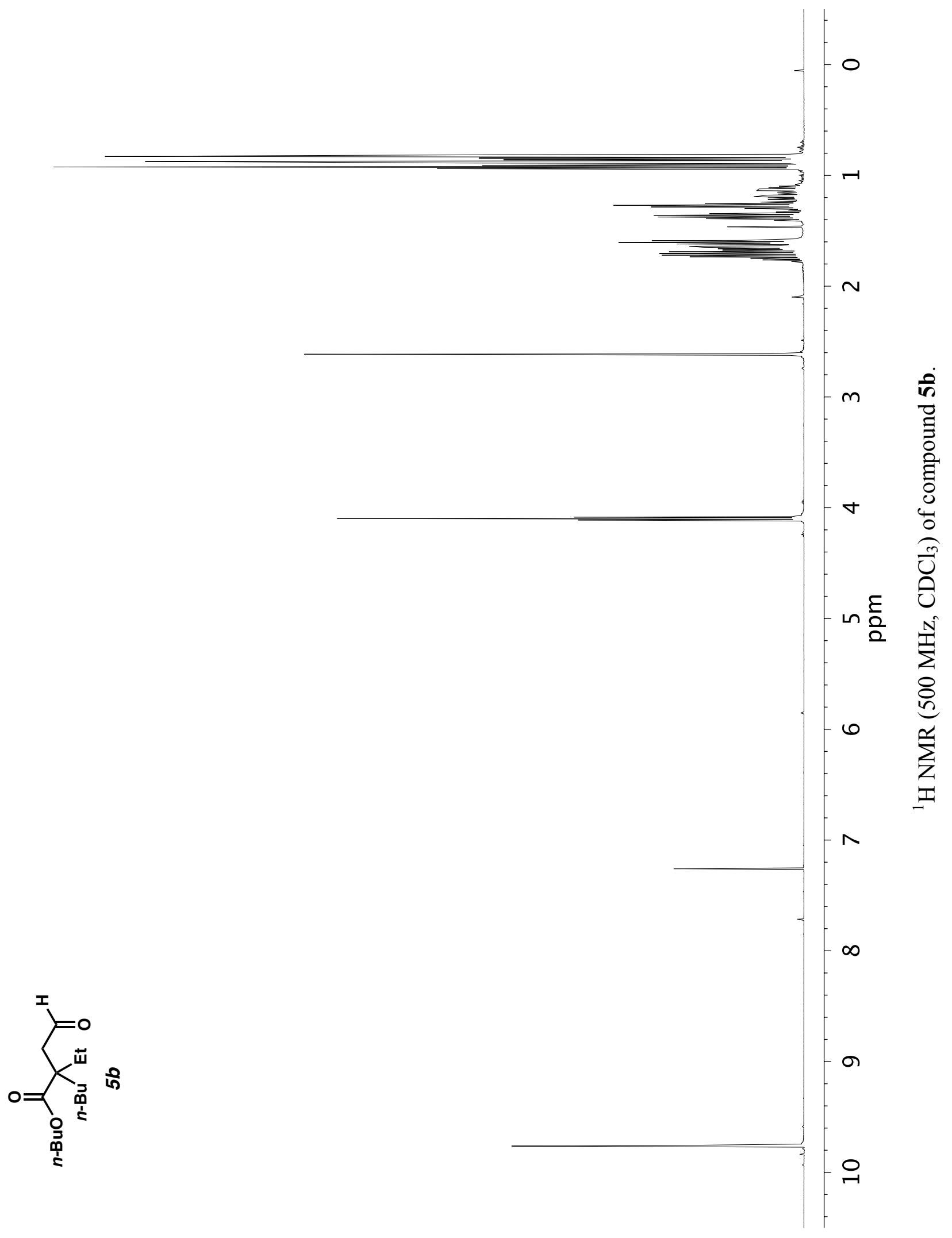



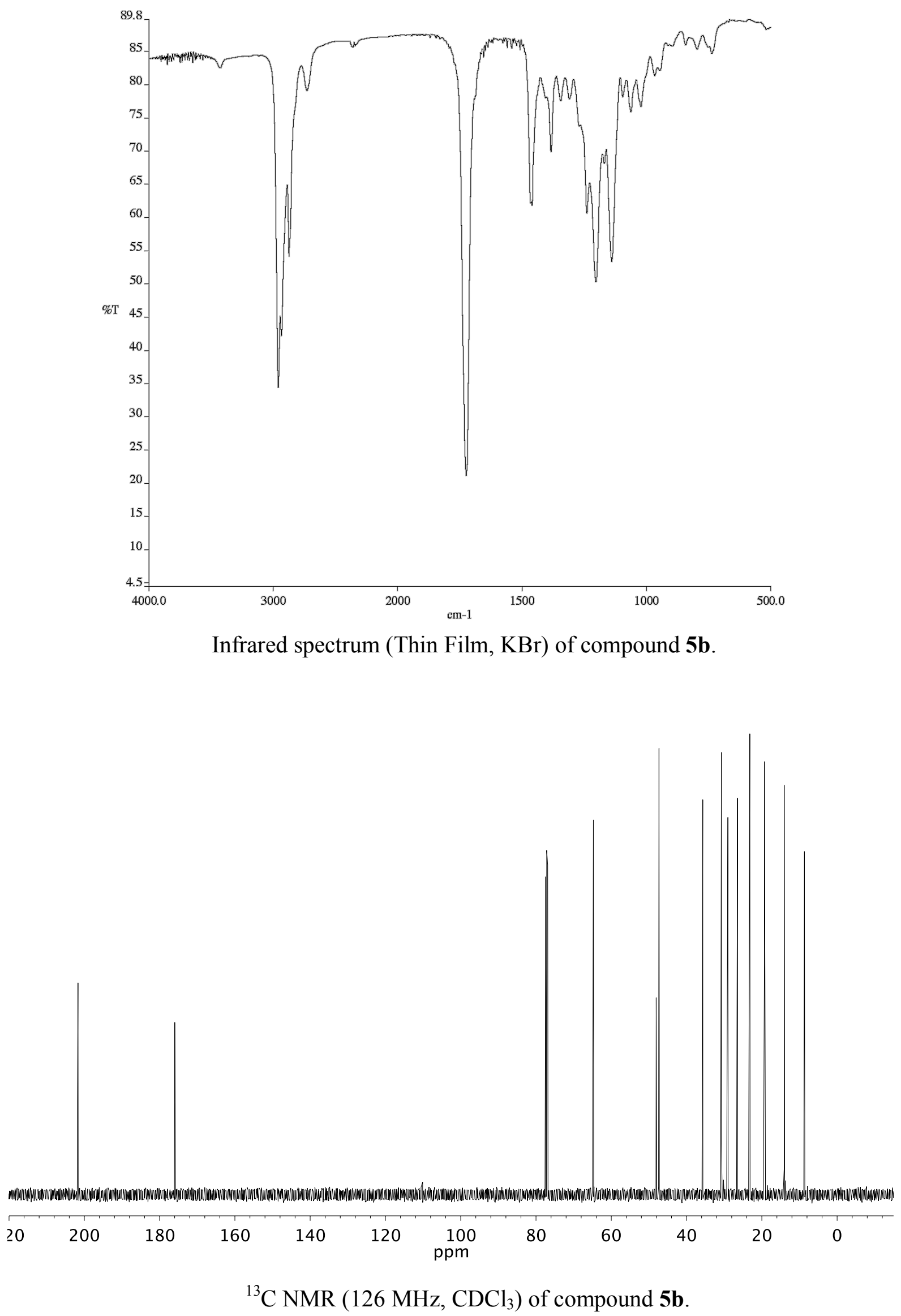


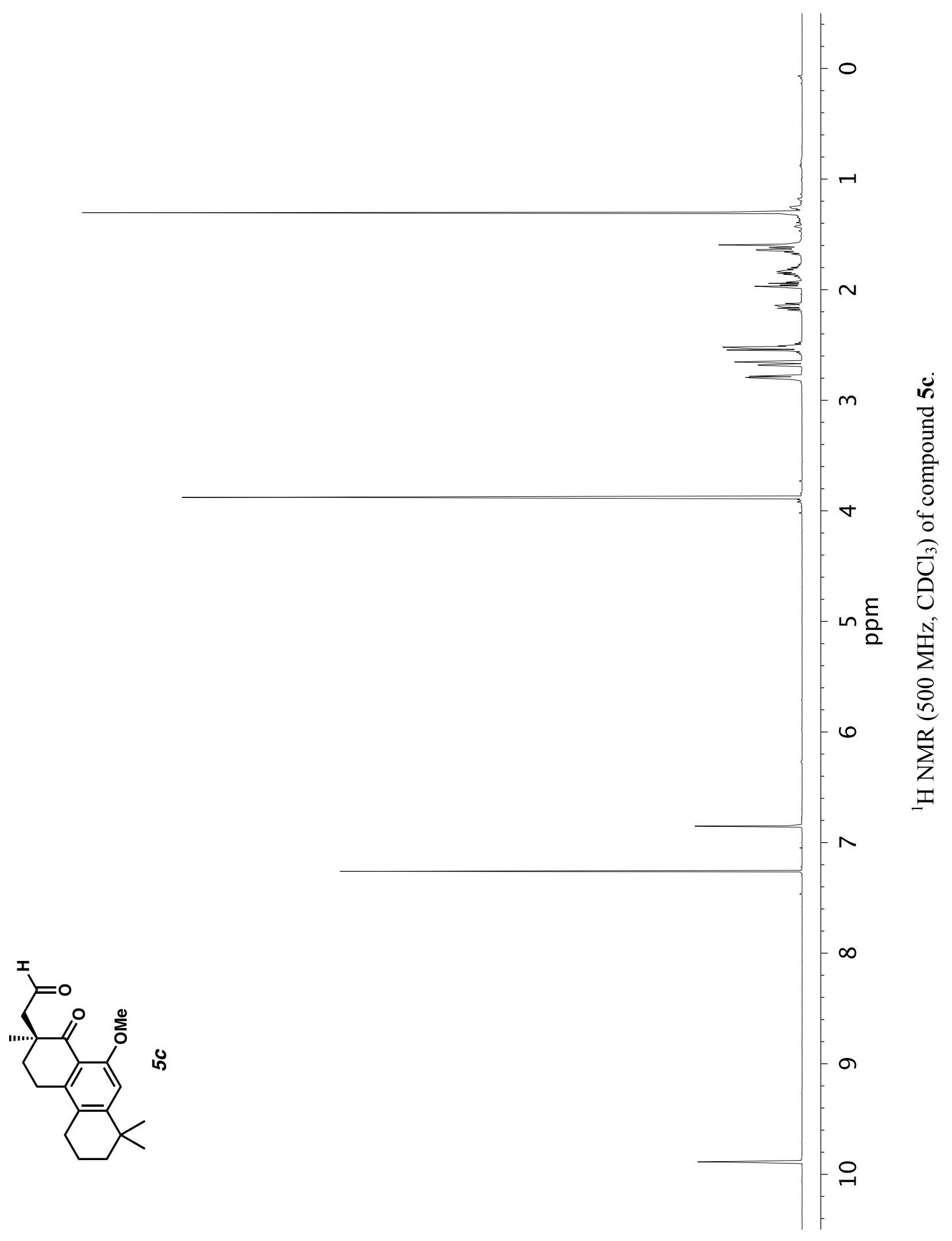




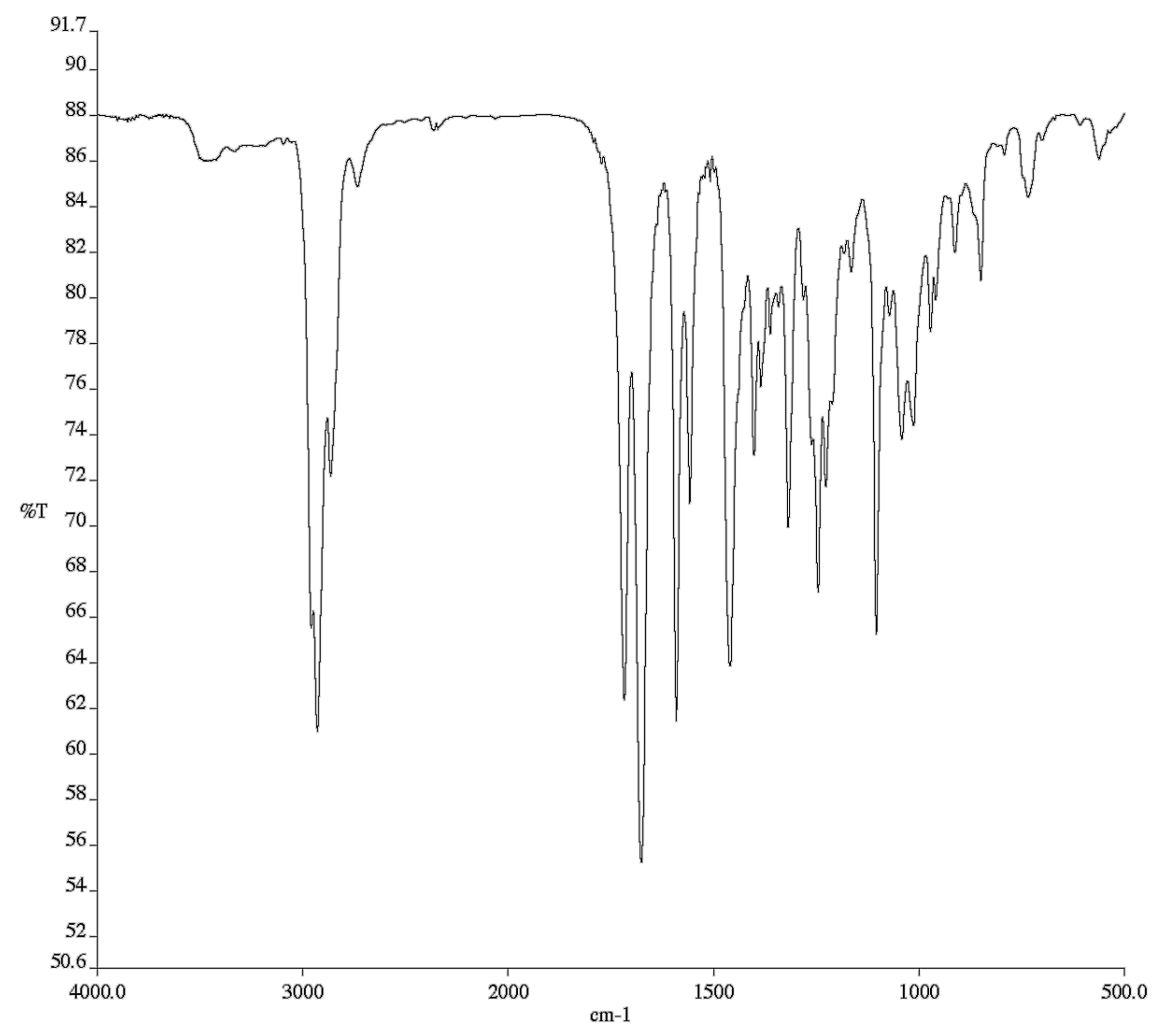

Infrared spectrum (Thin Film, KBr) of compound 5c.

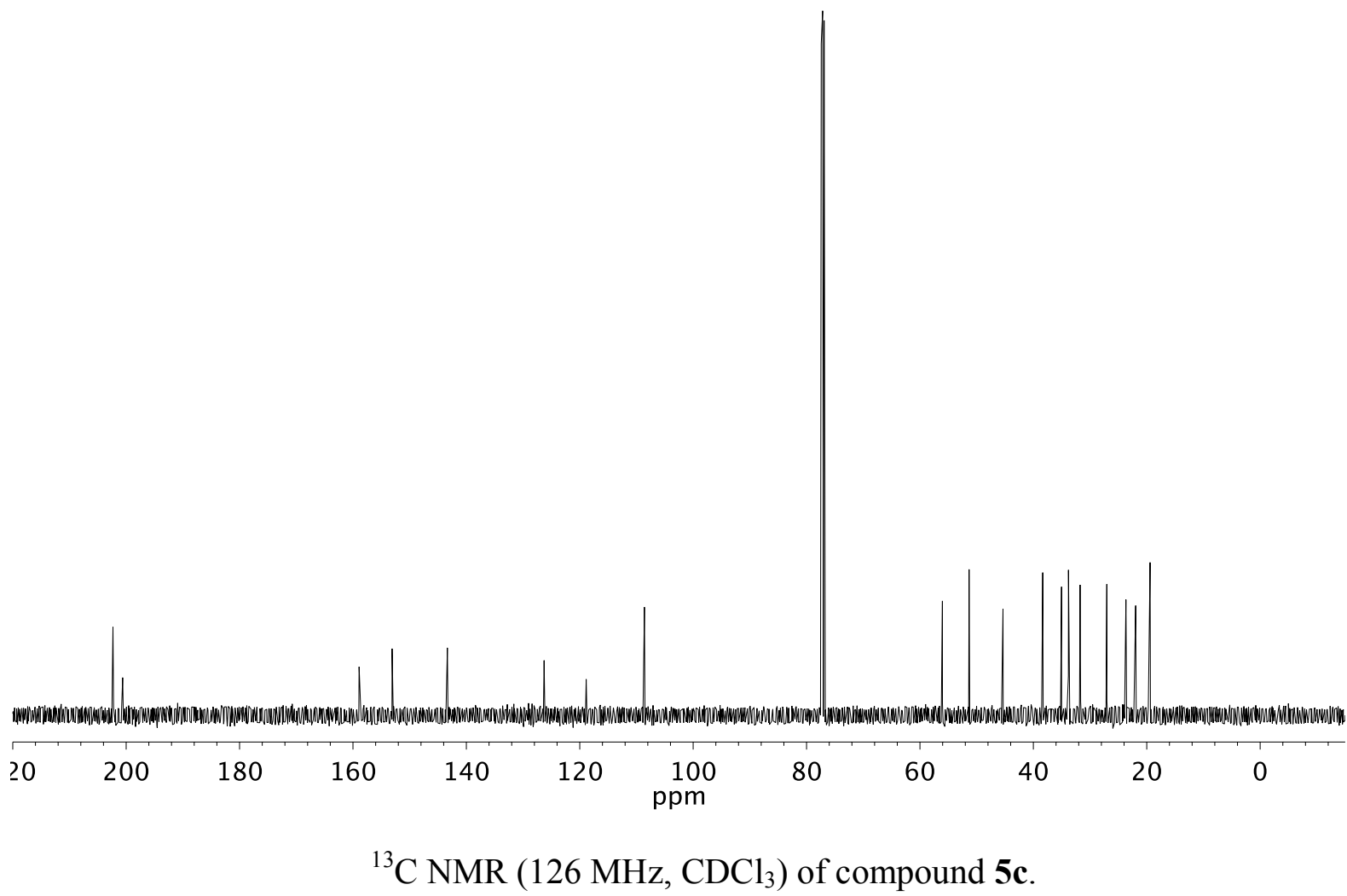




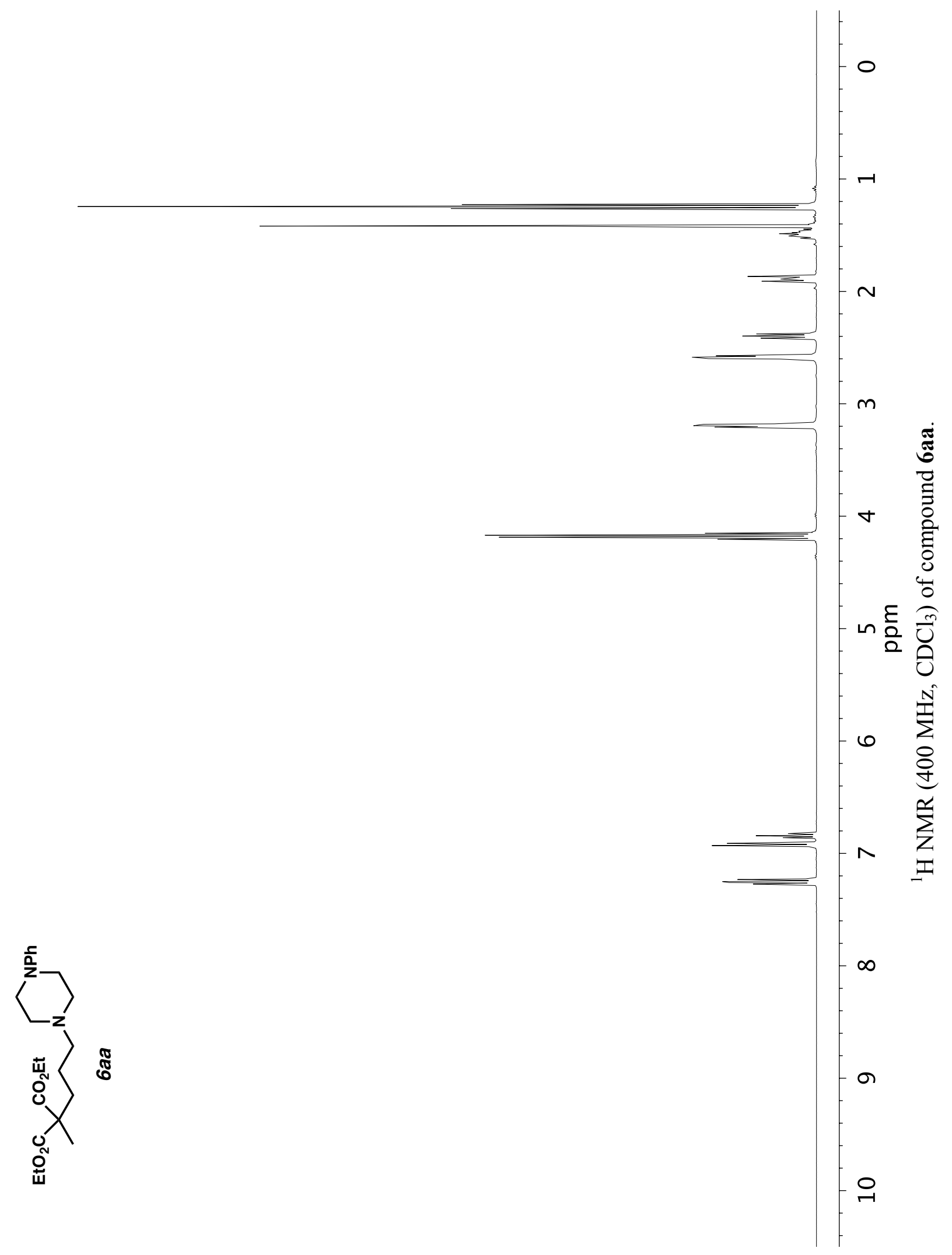



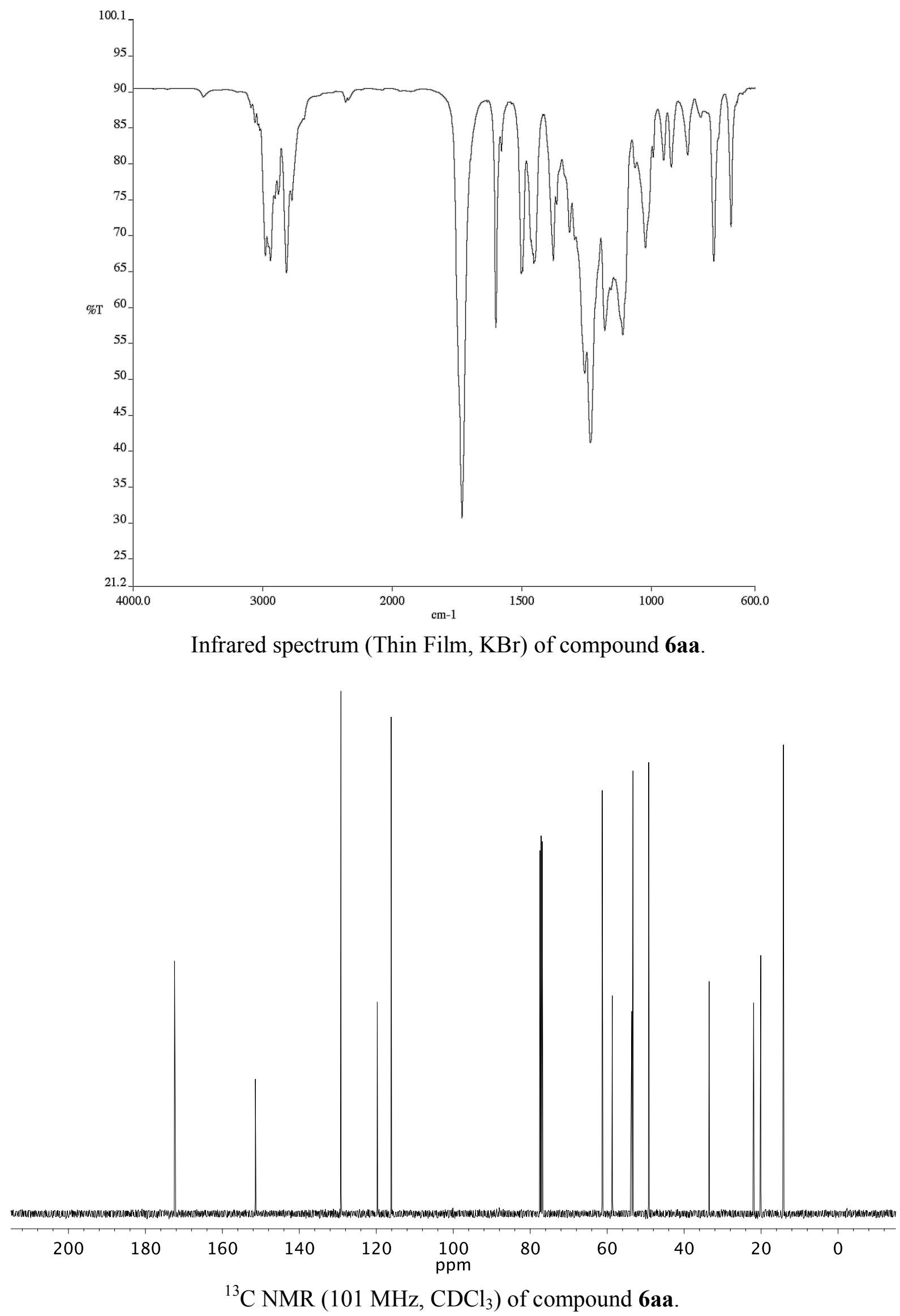


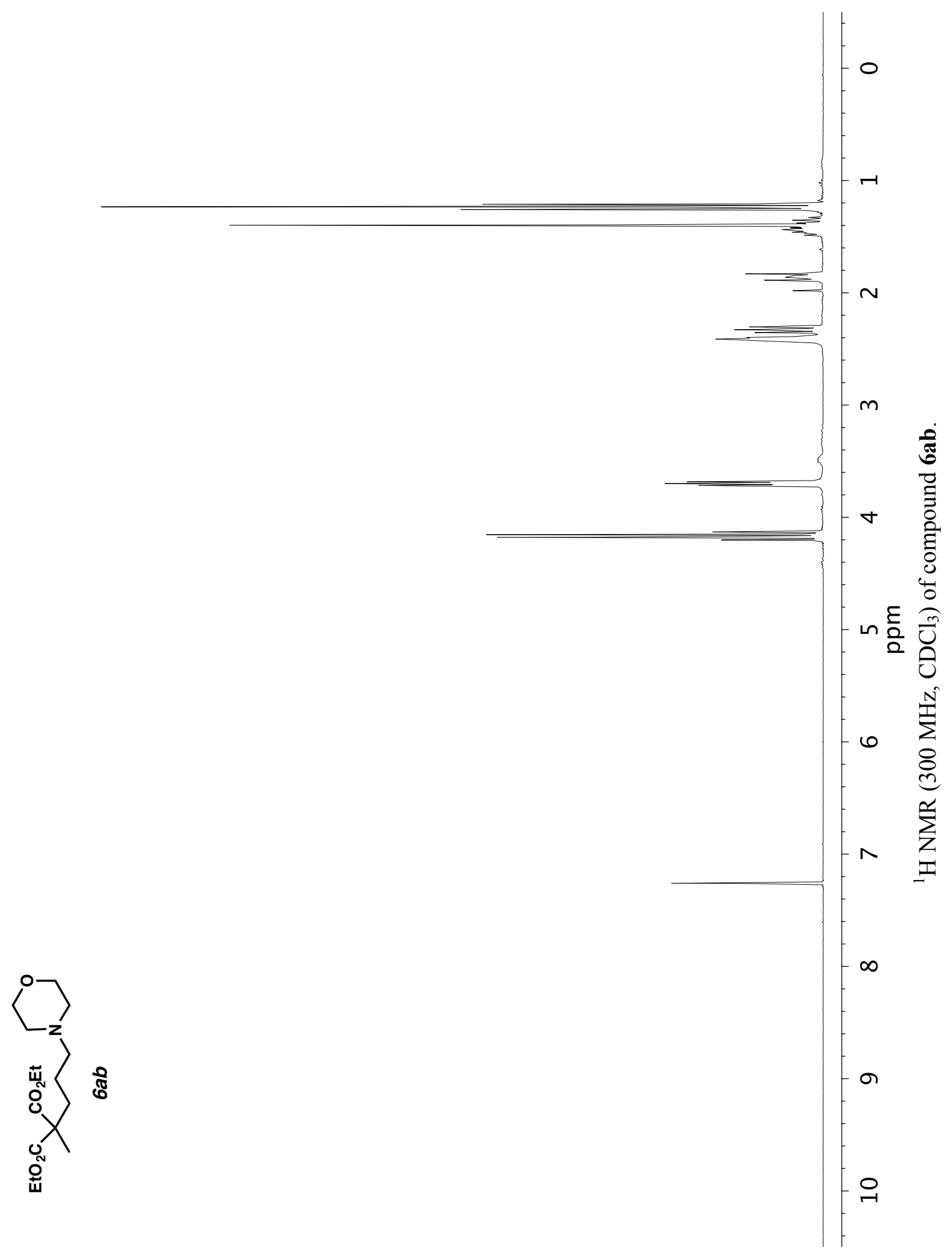



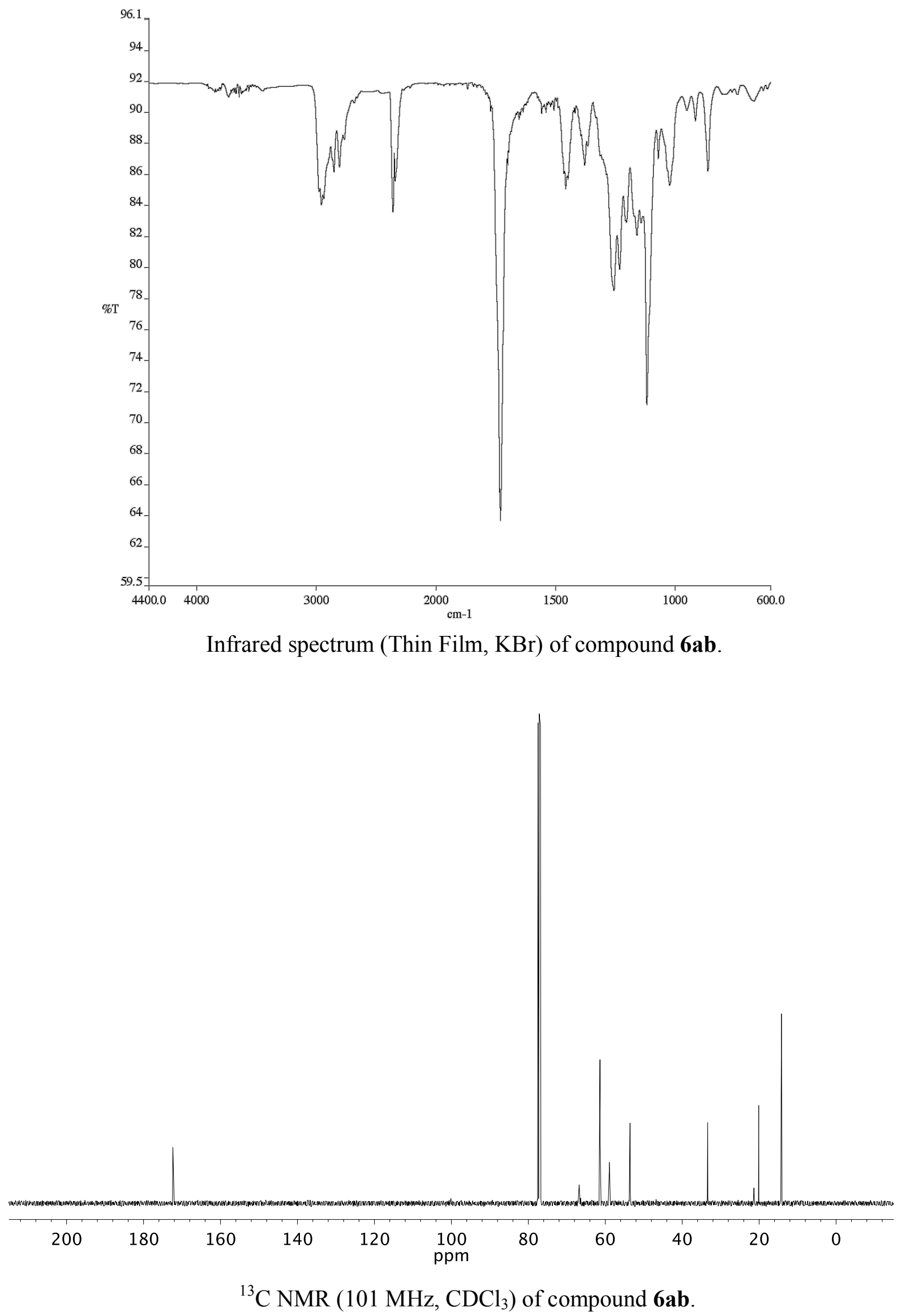


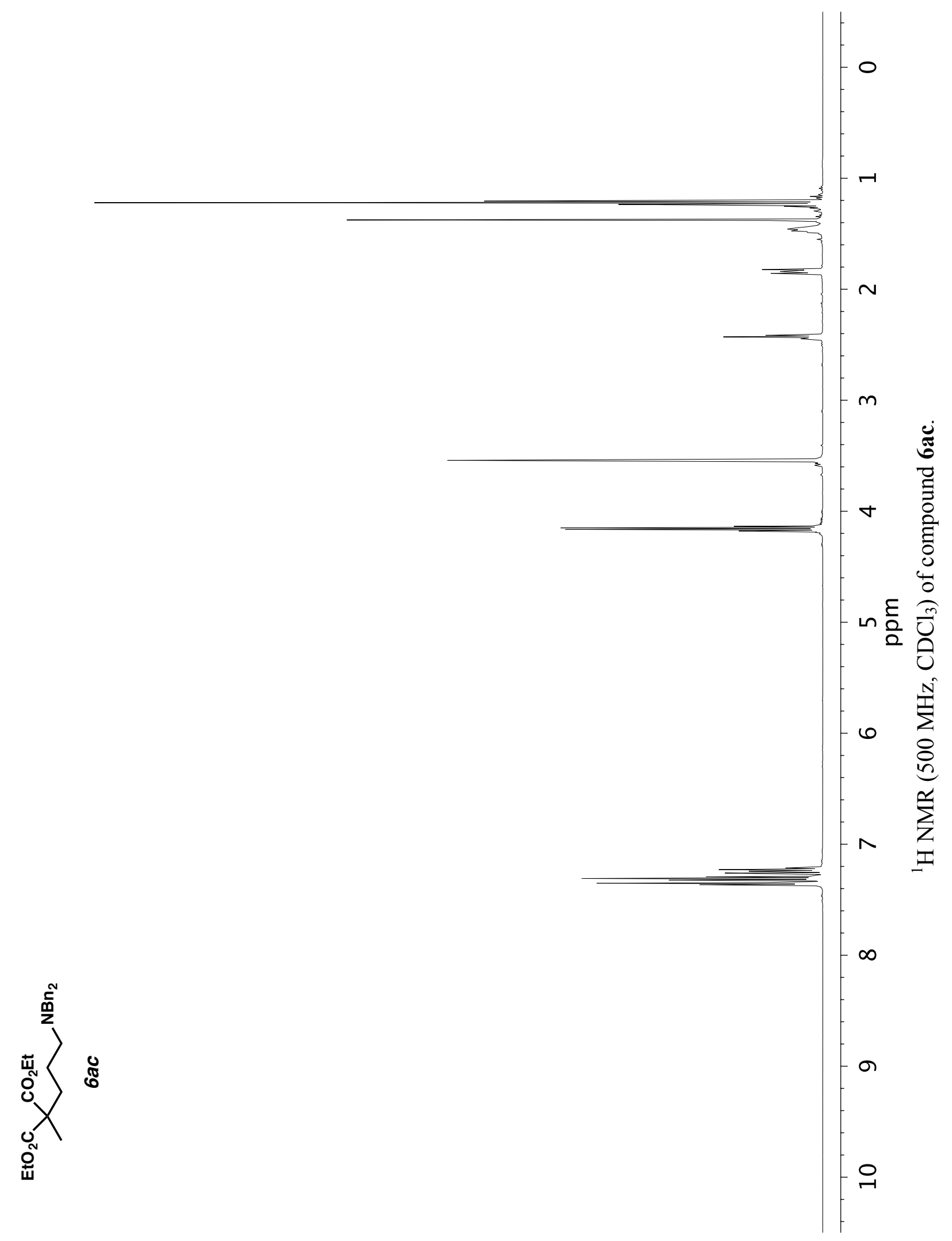




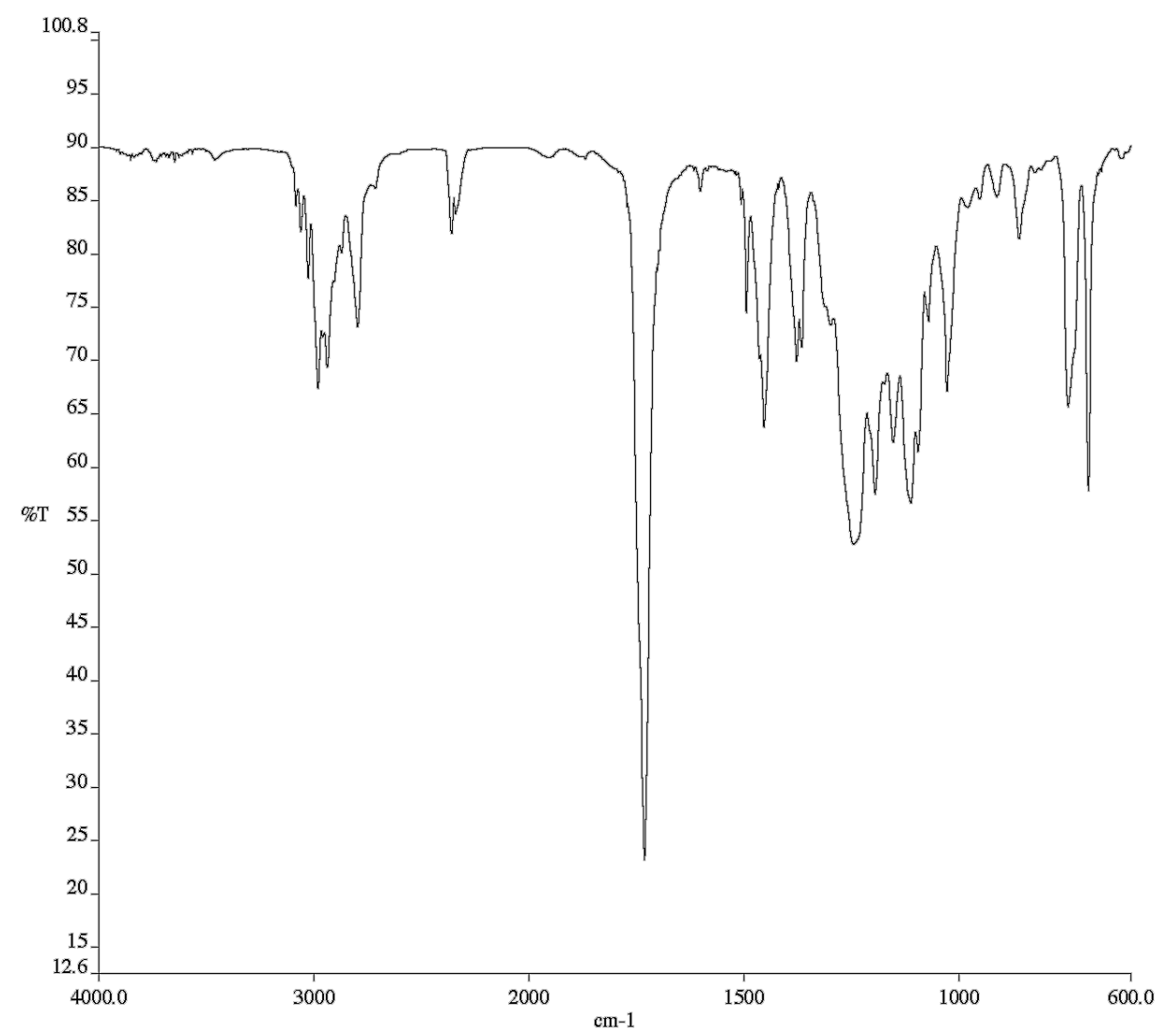

Infrared spectrum (Thin Film, KBr) of compound 6ac.

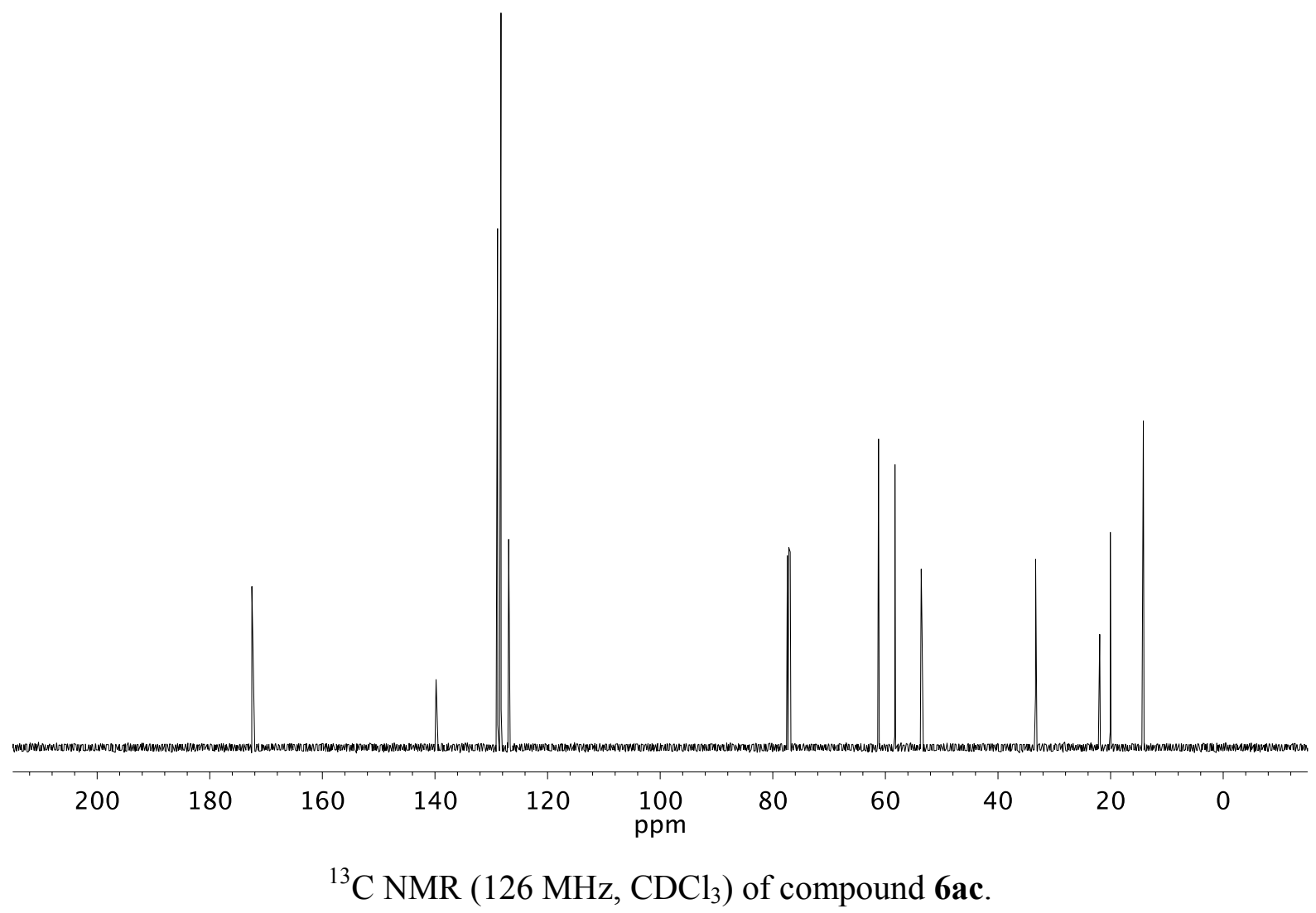




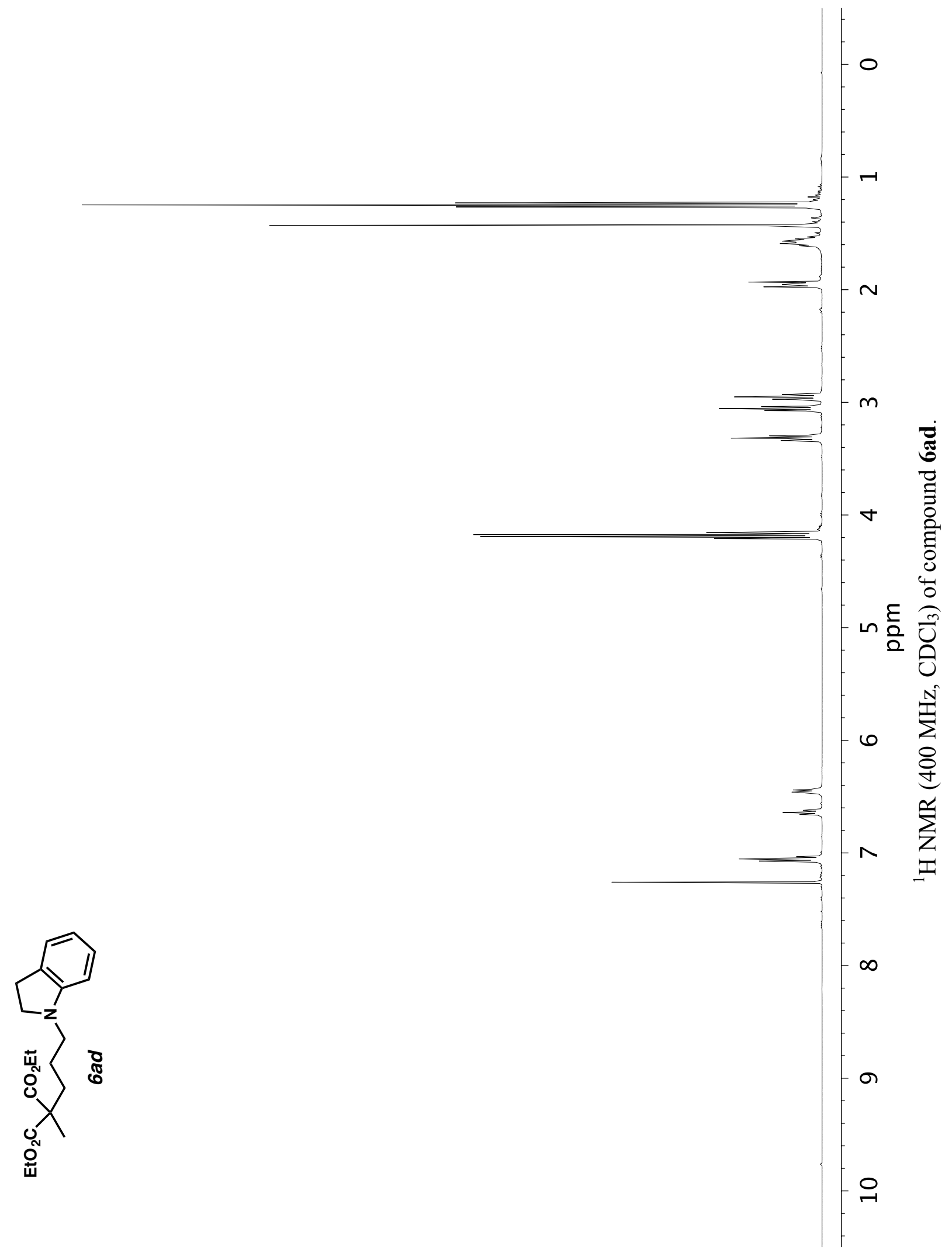



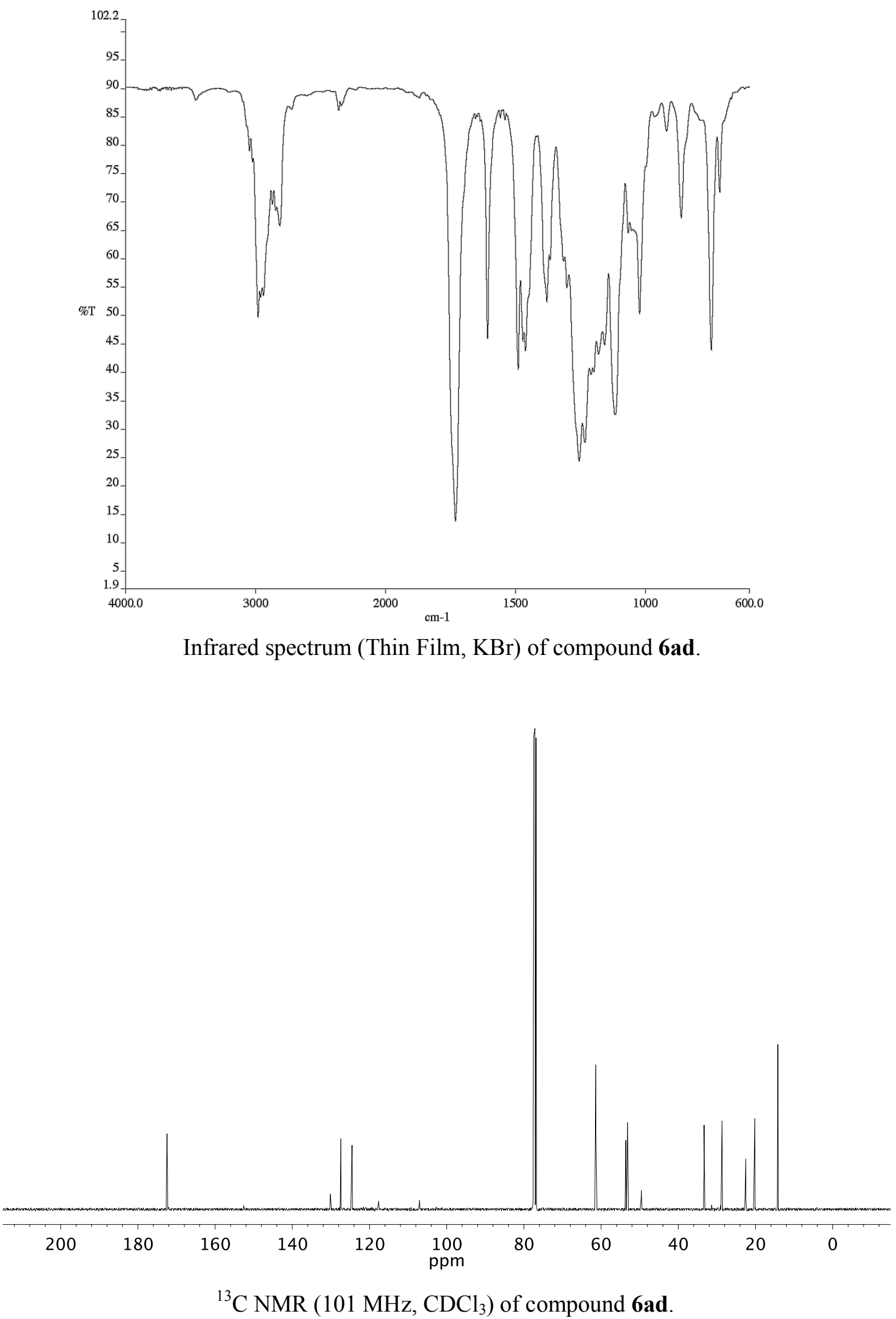


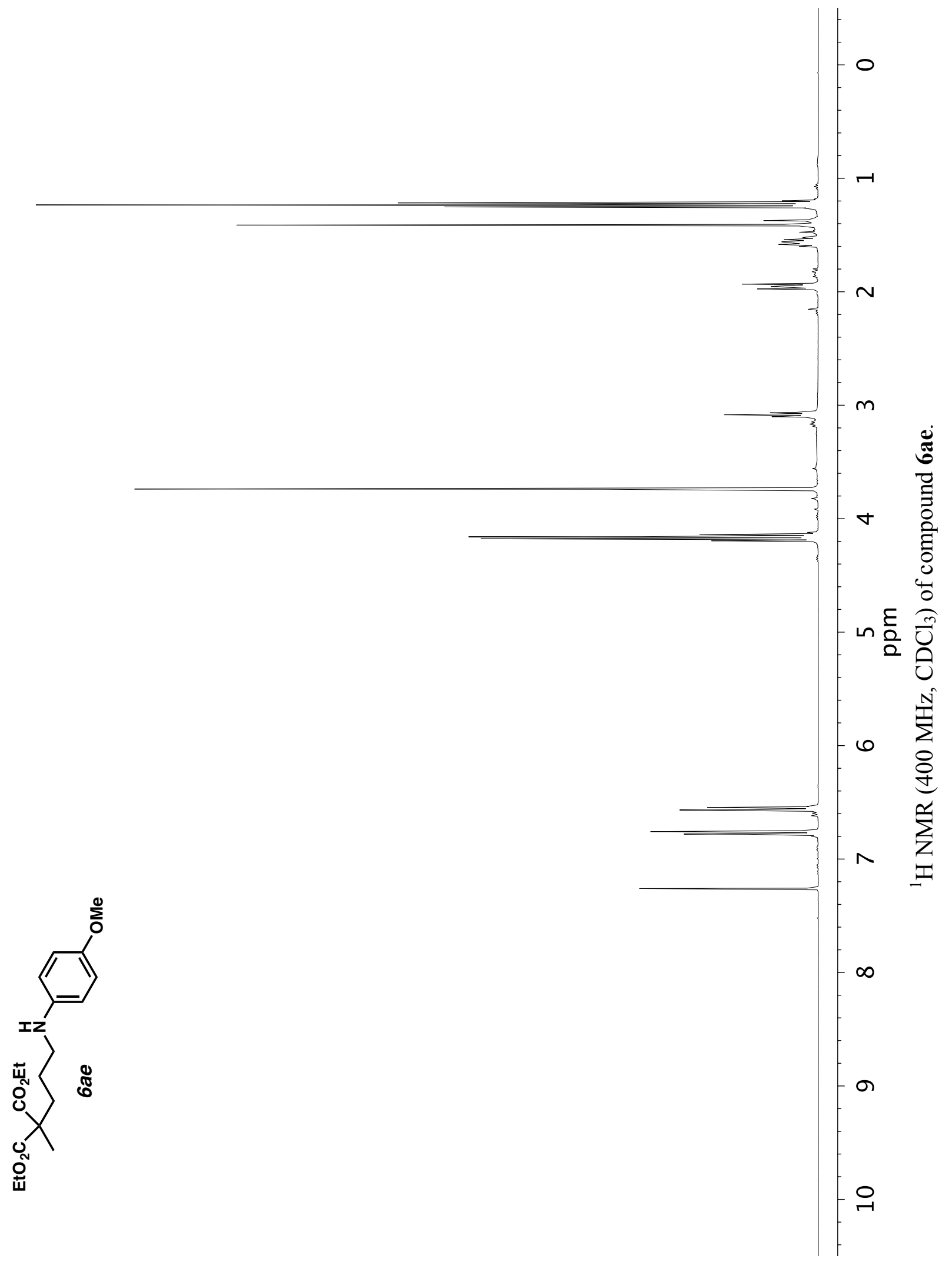




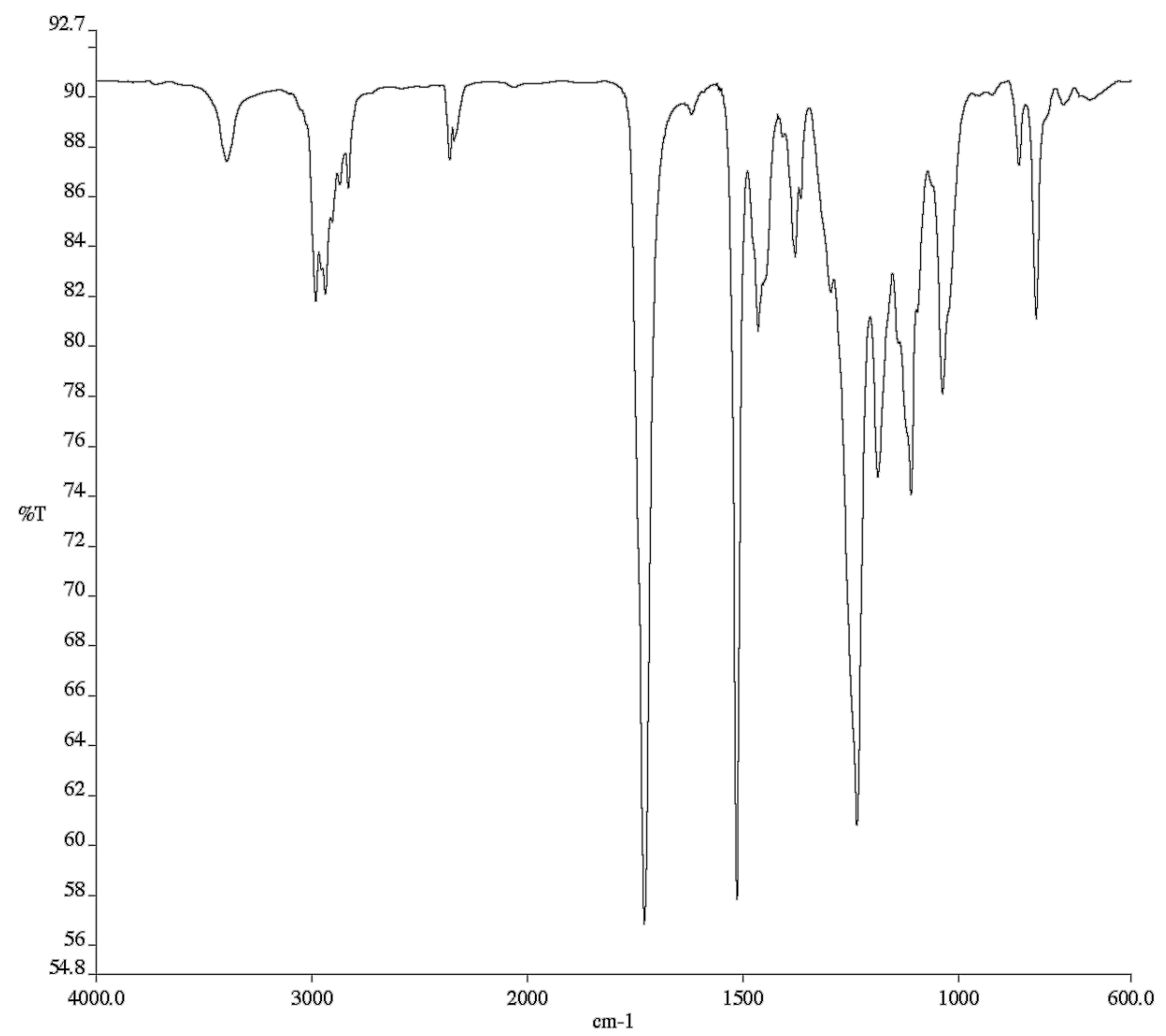

Infrared spectrum (Thin Film, KBr) of compound 6ae.

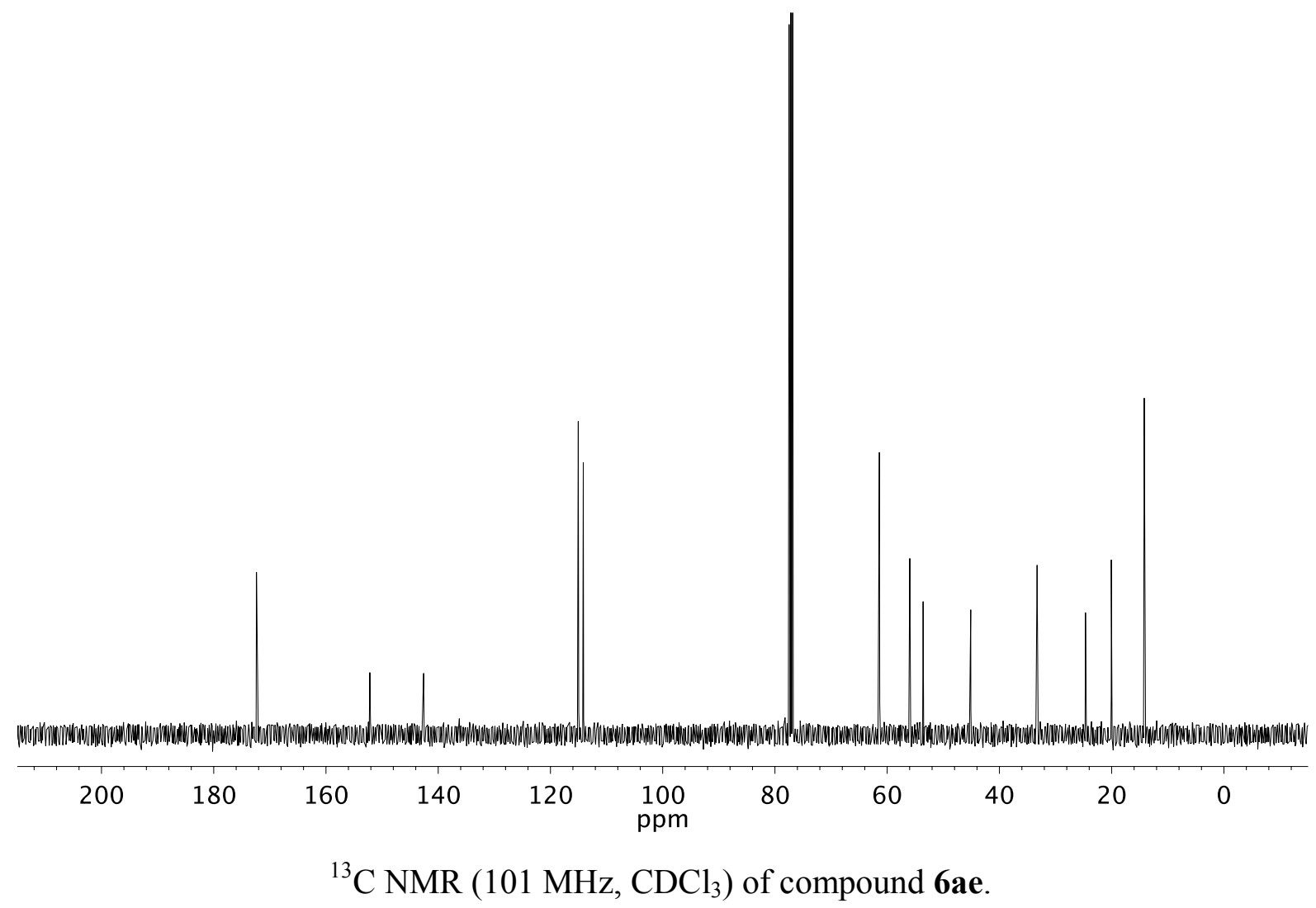




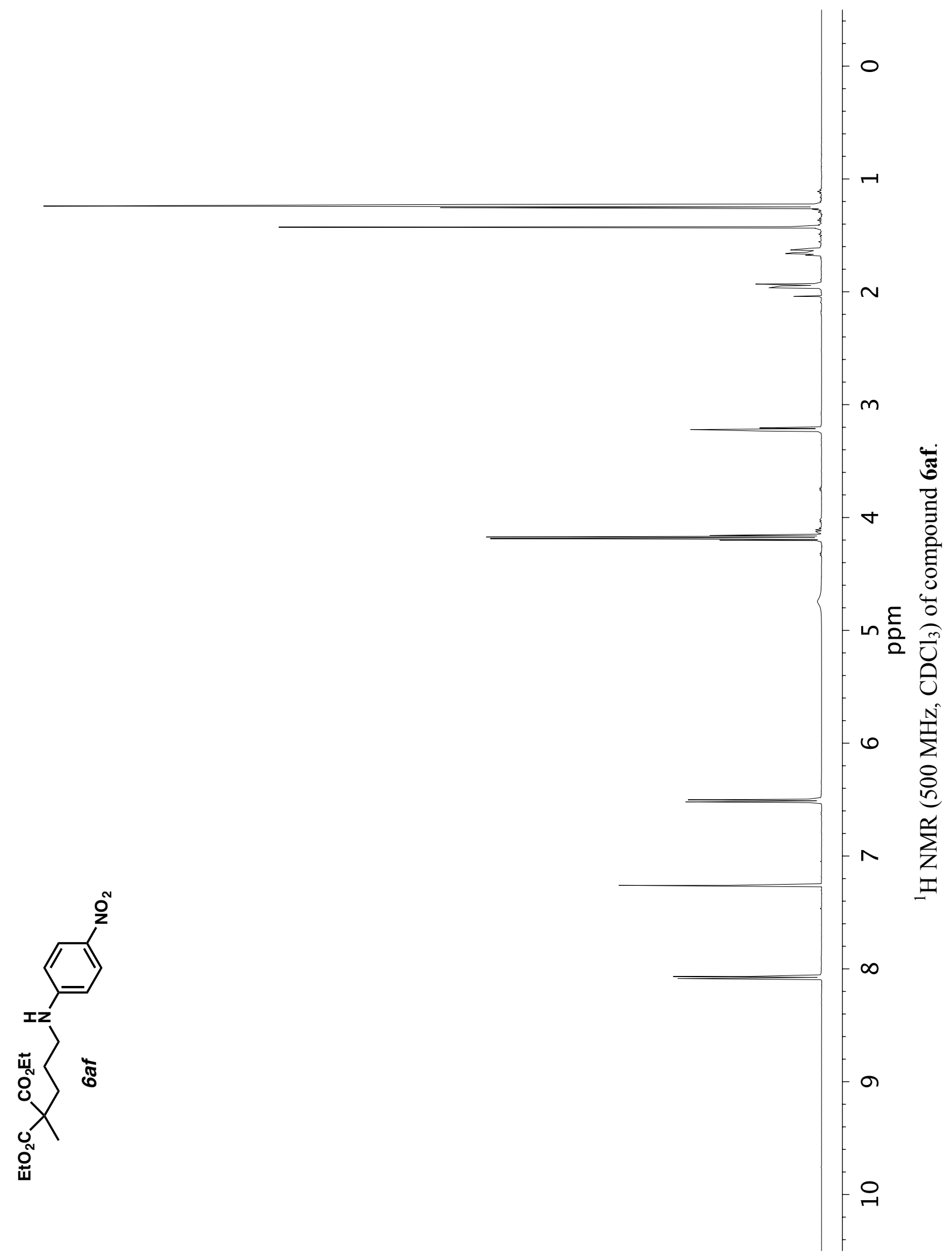



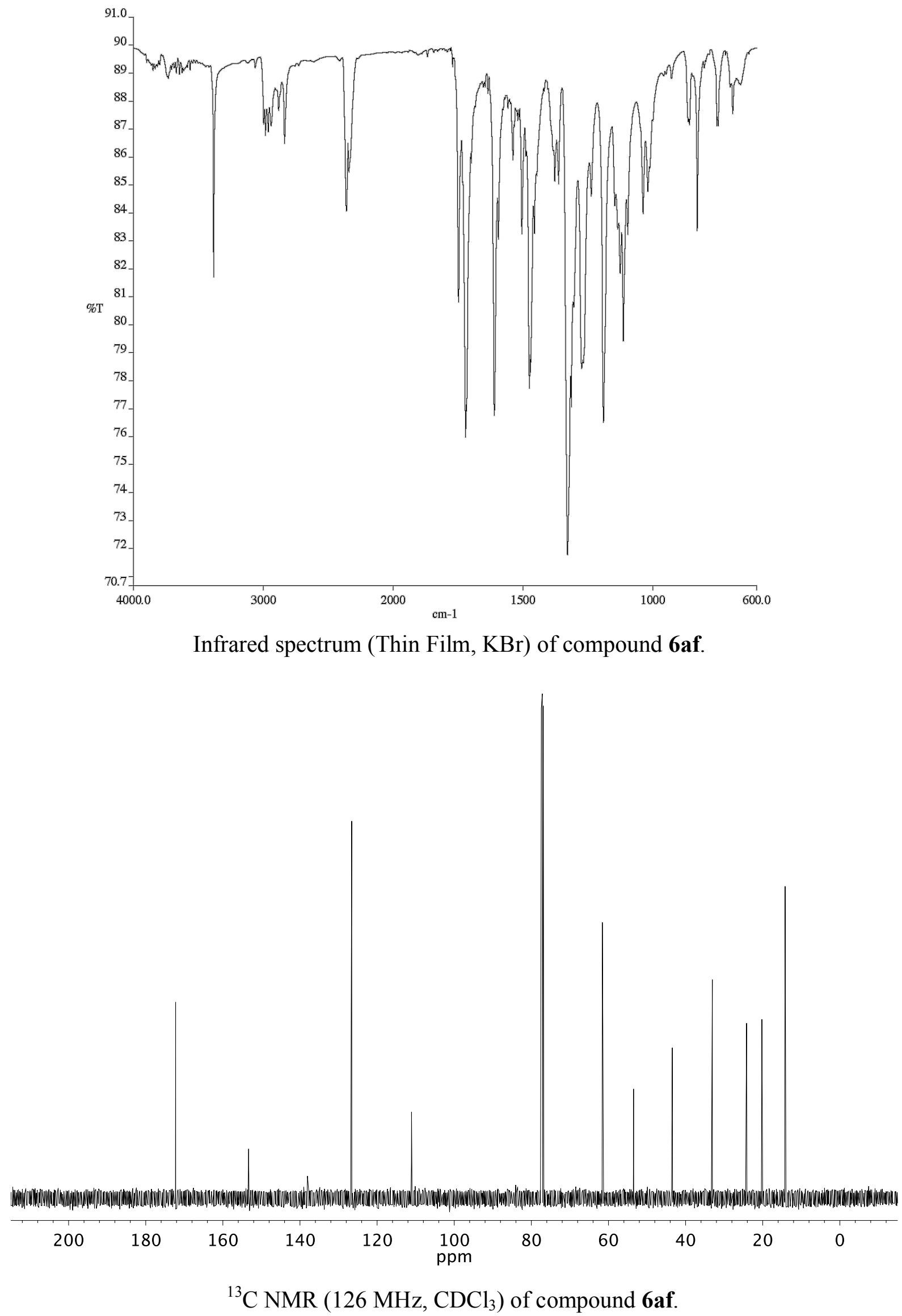


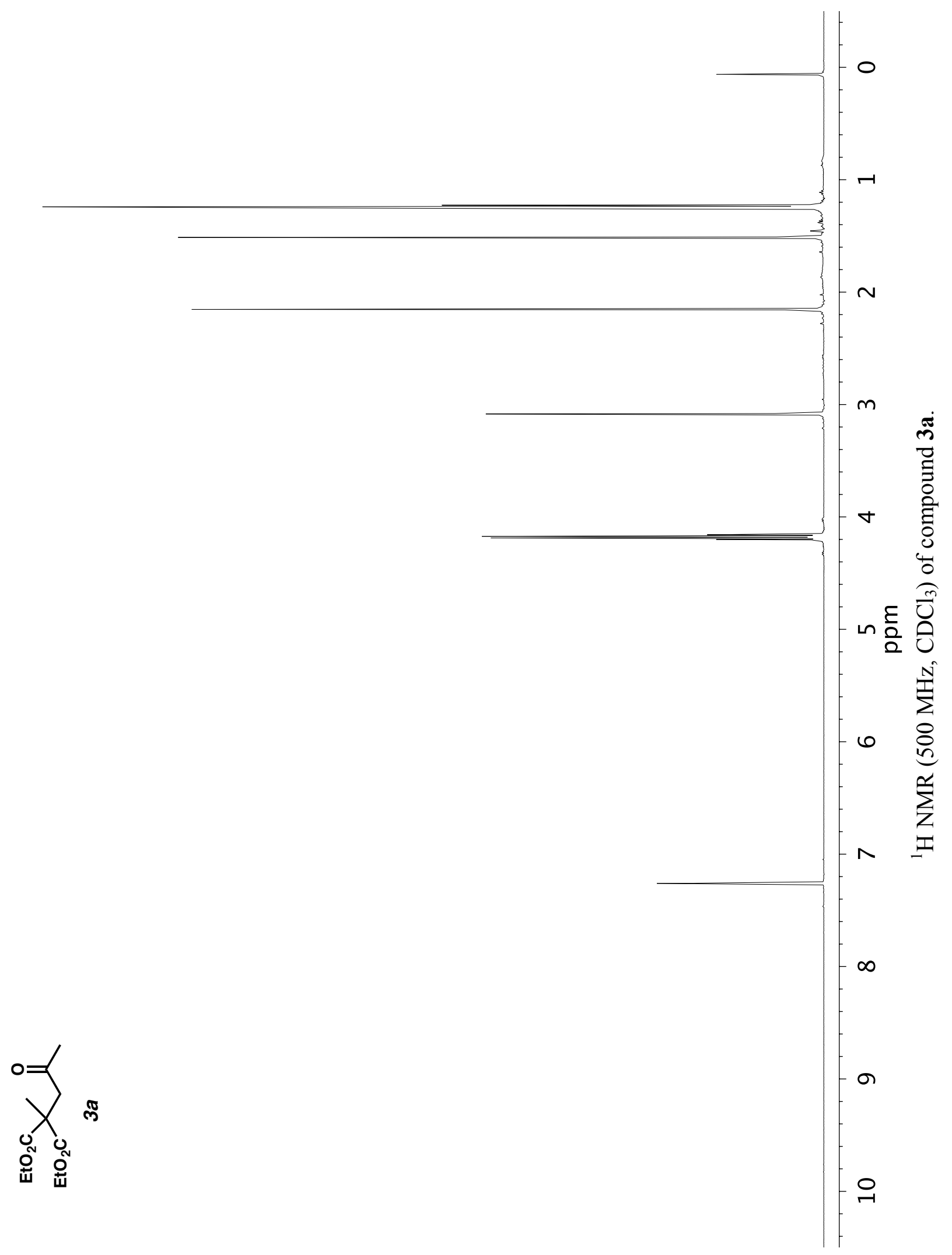



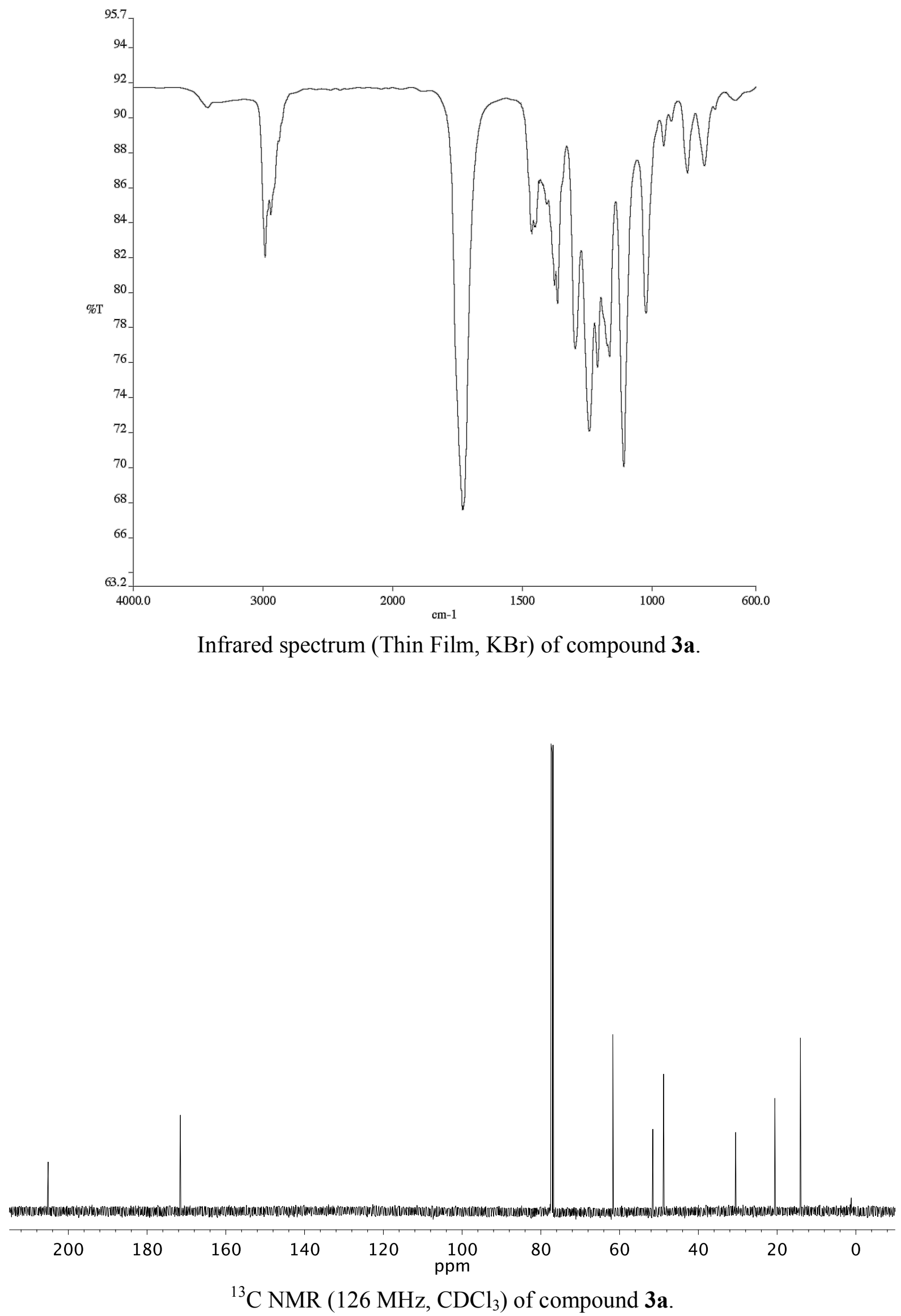


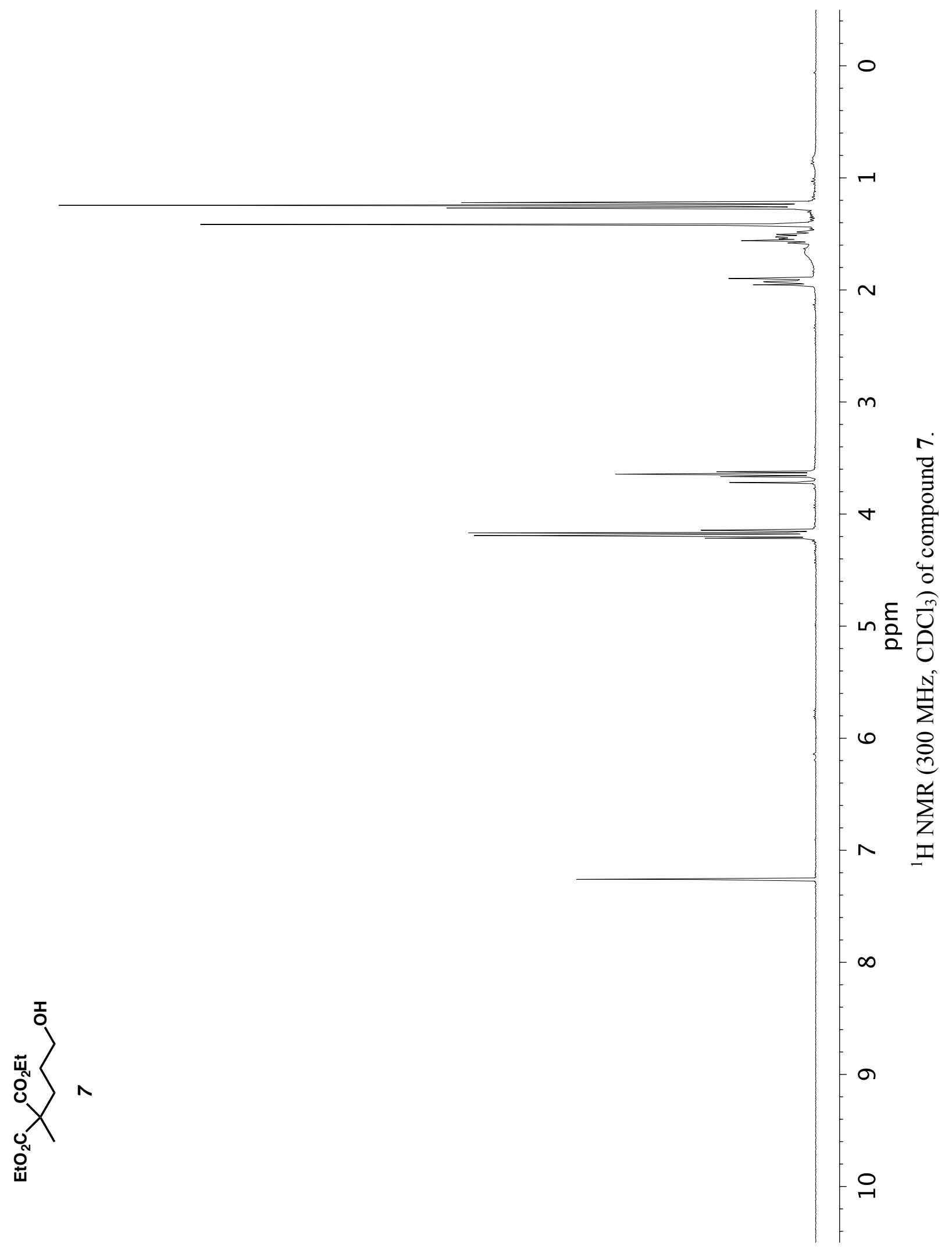



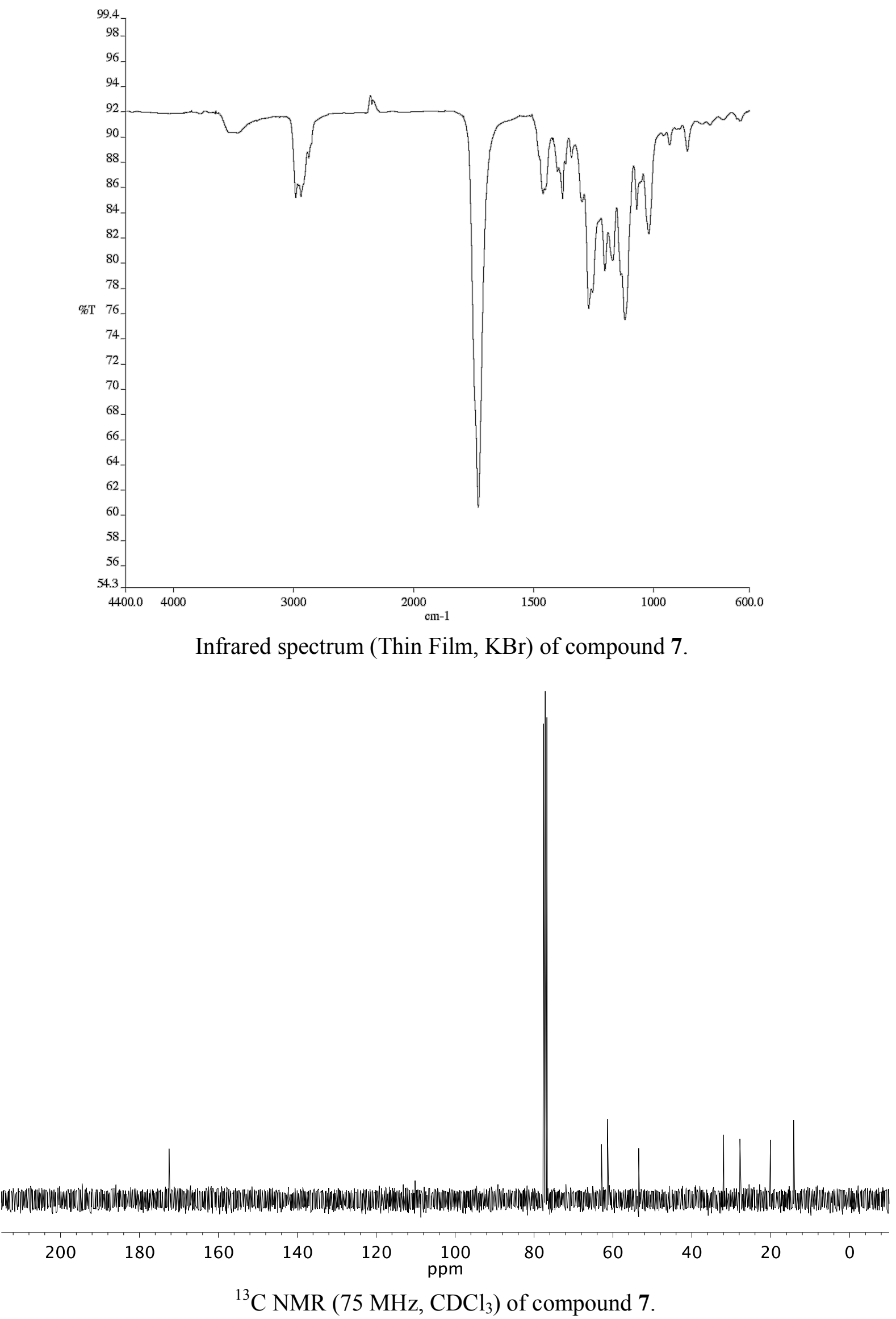


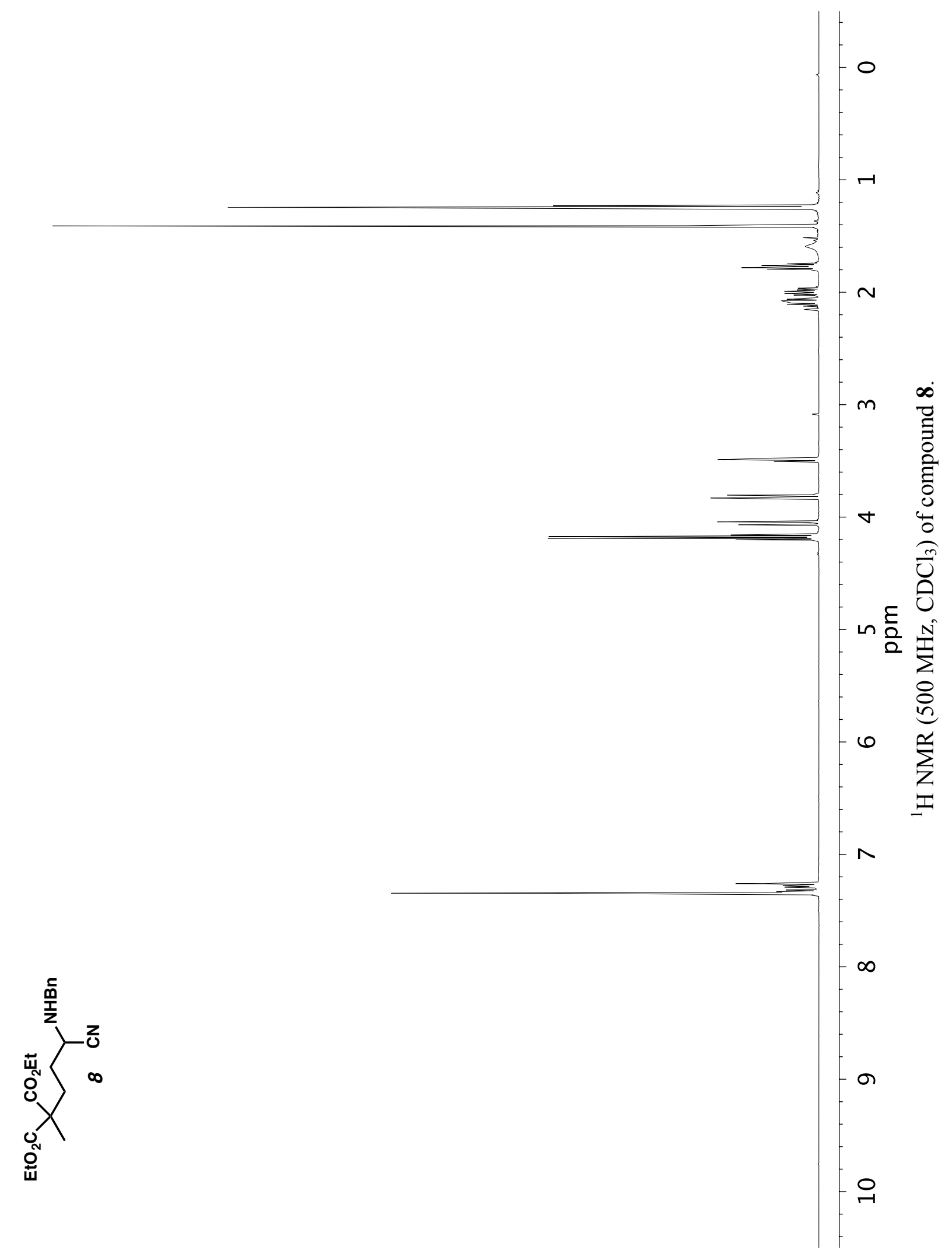



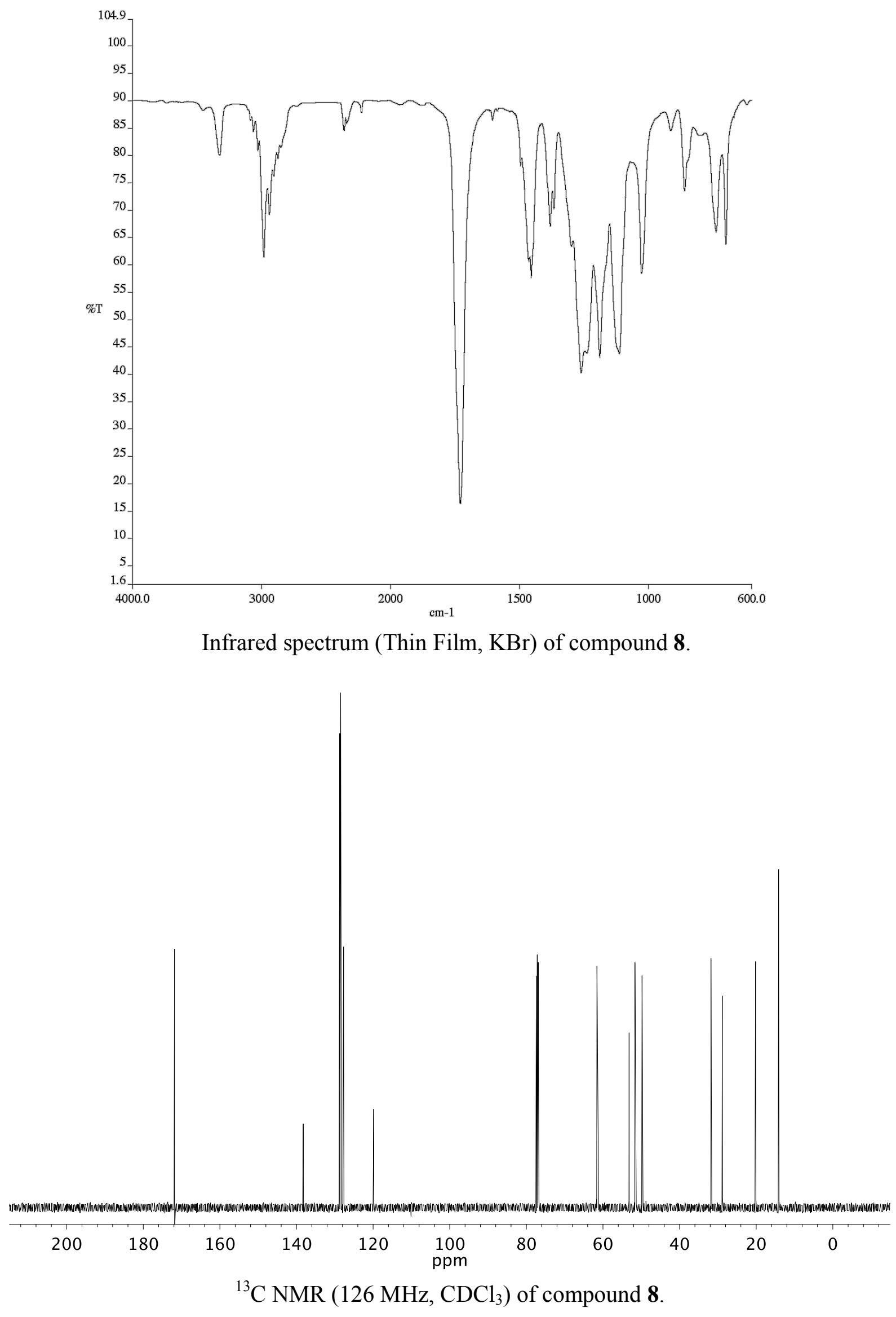


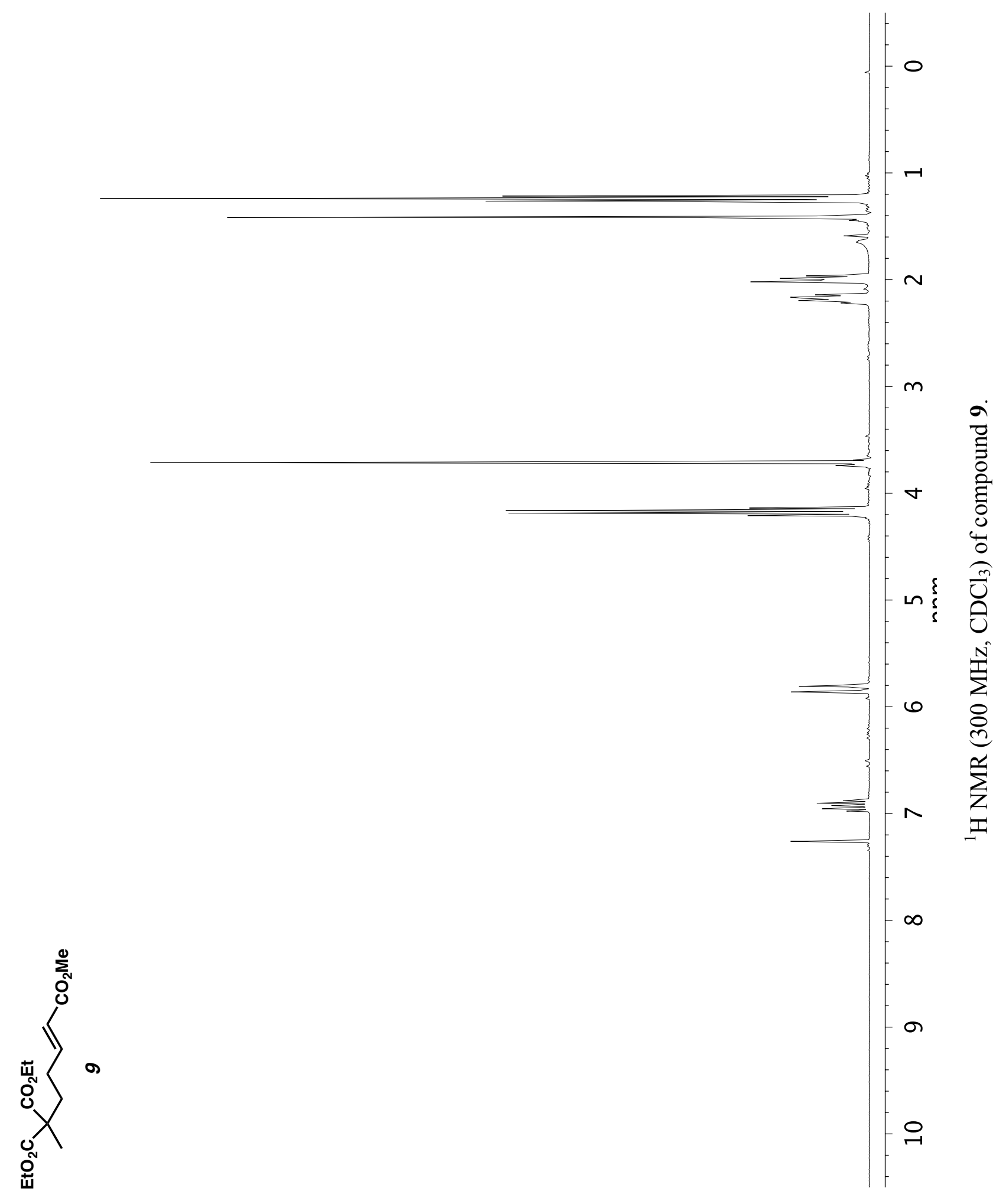



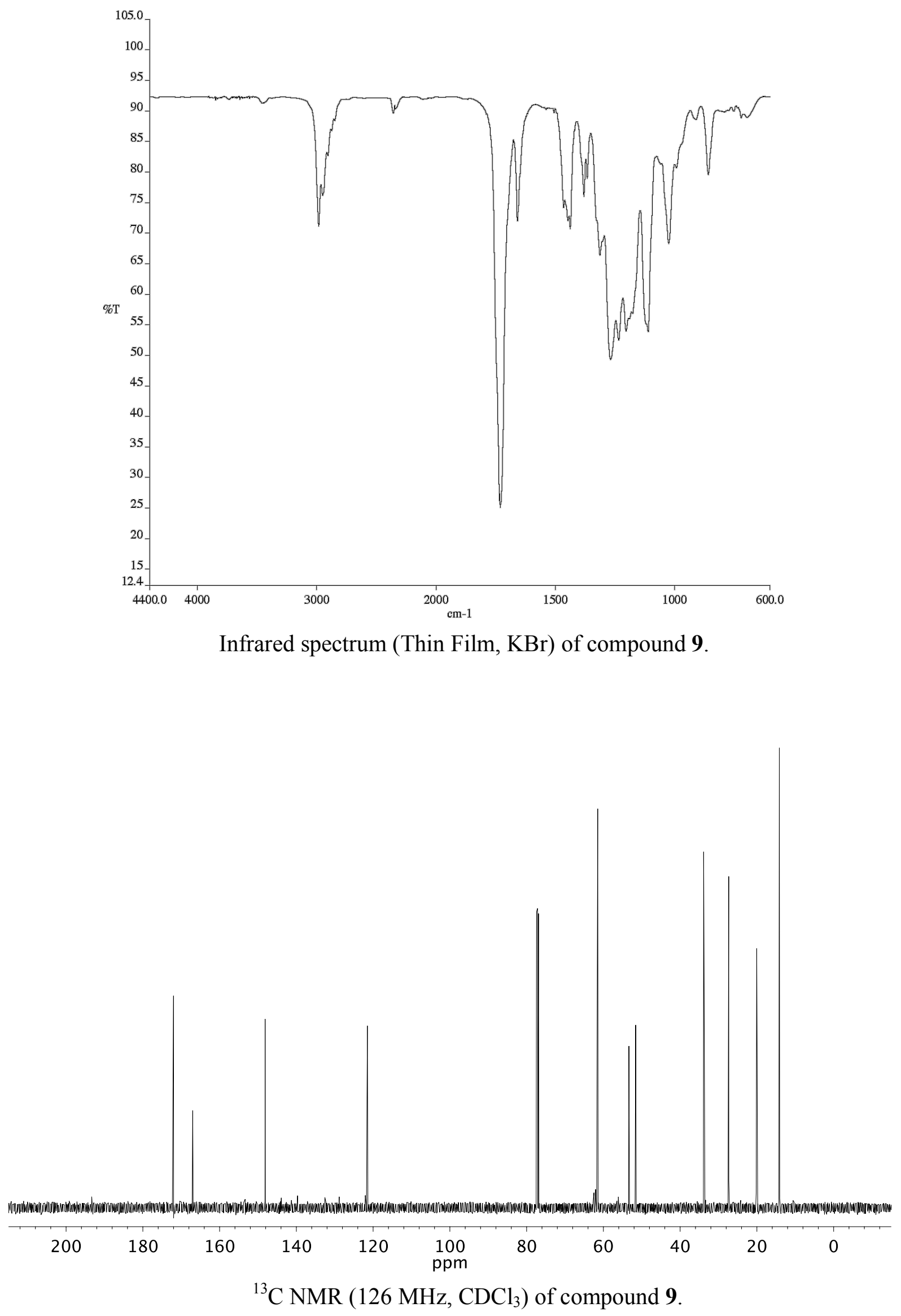


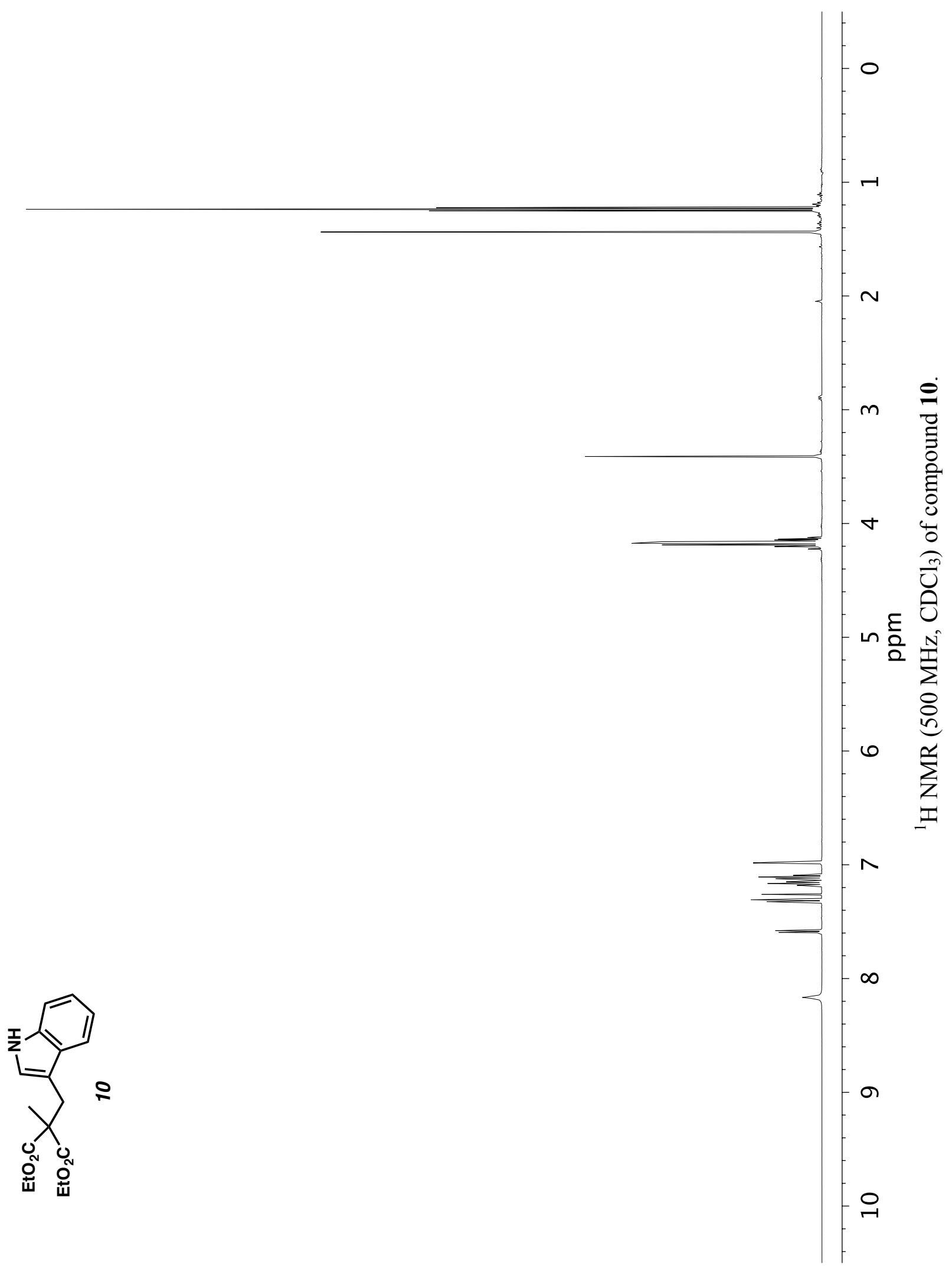



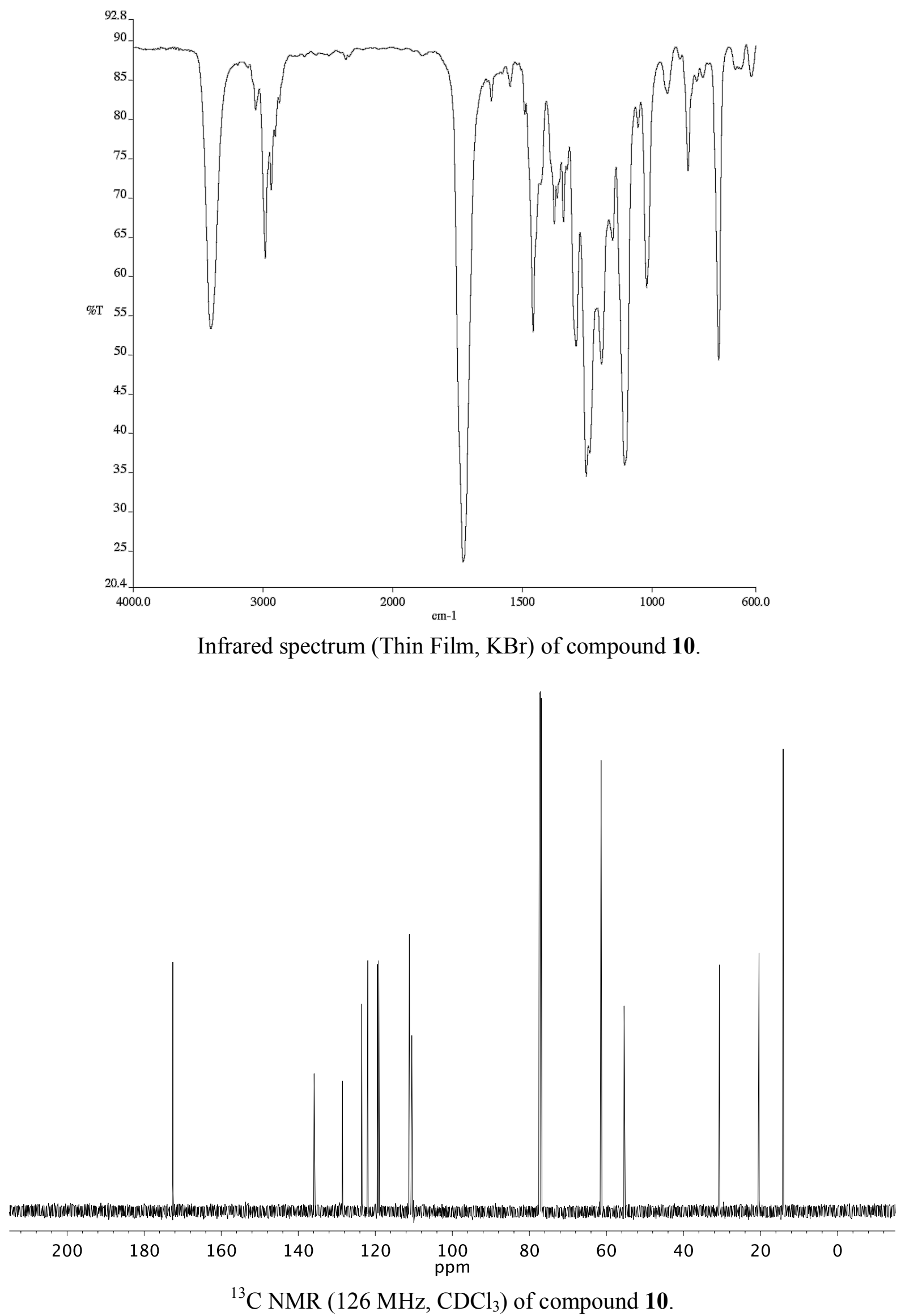


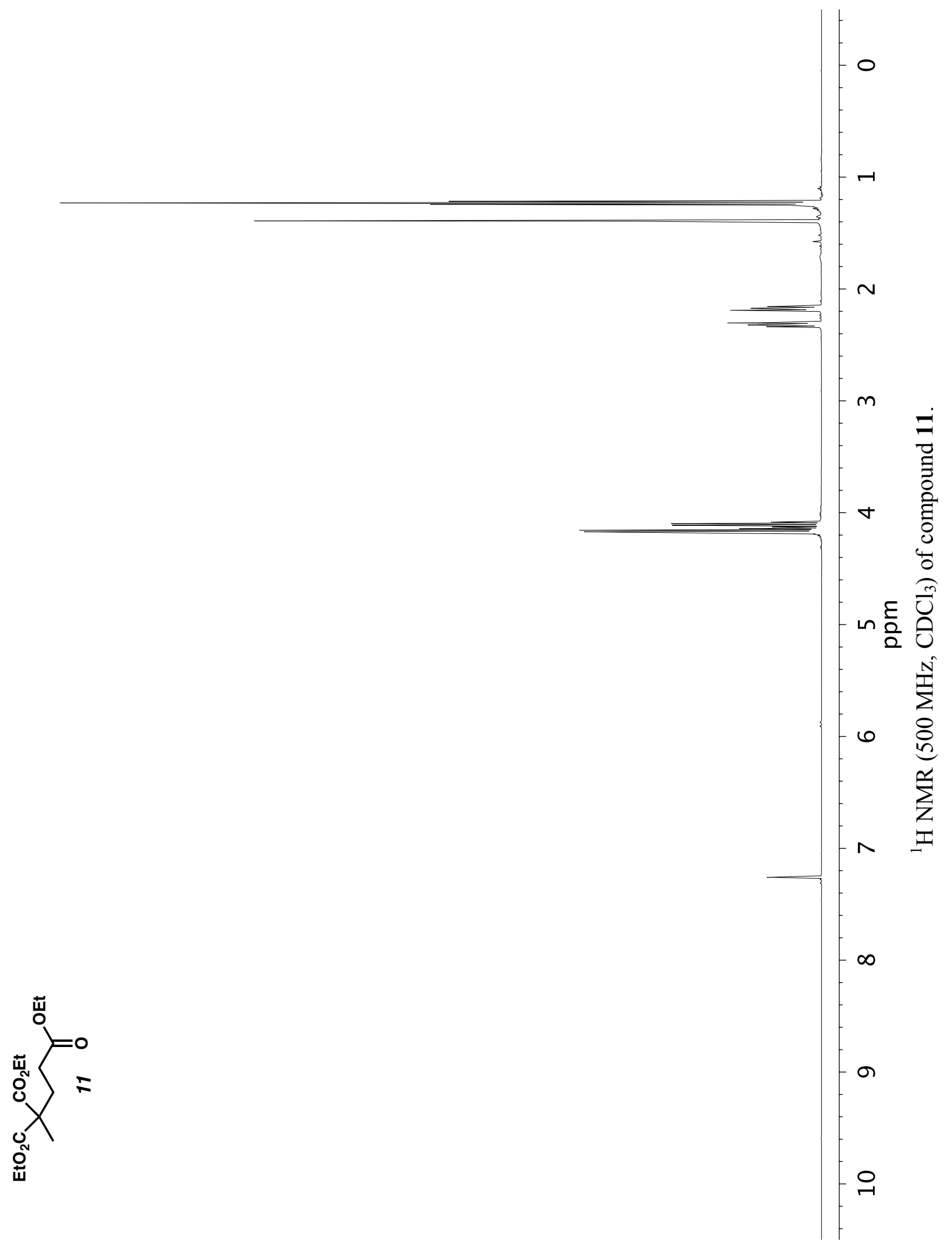



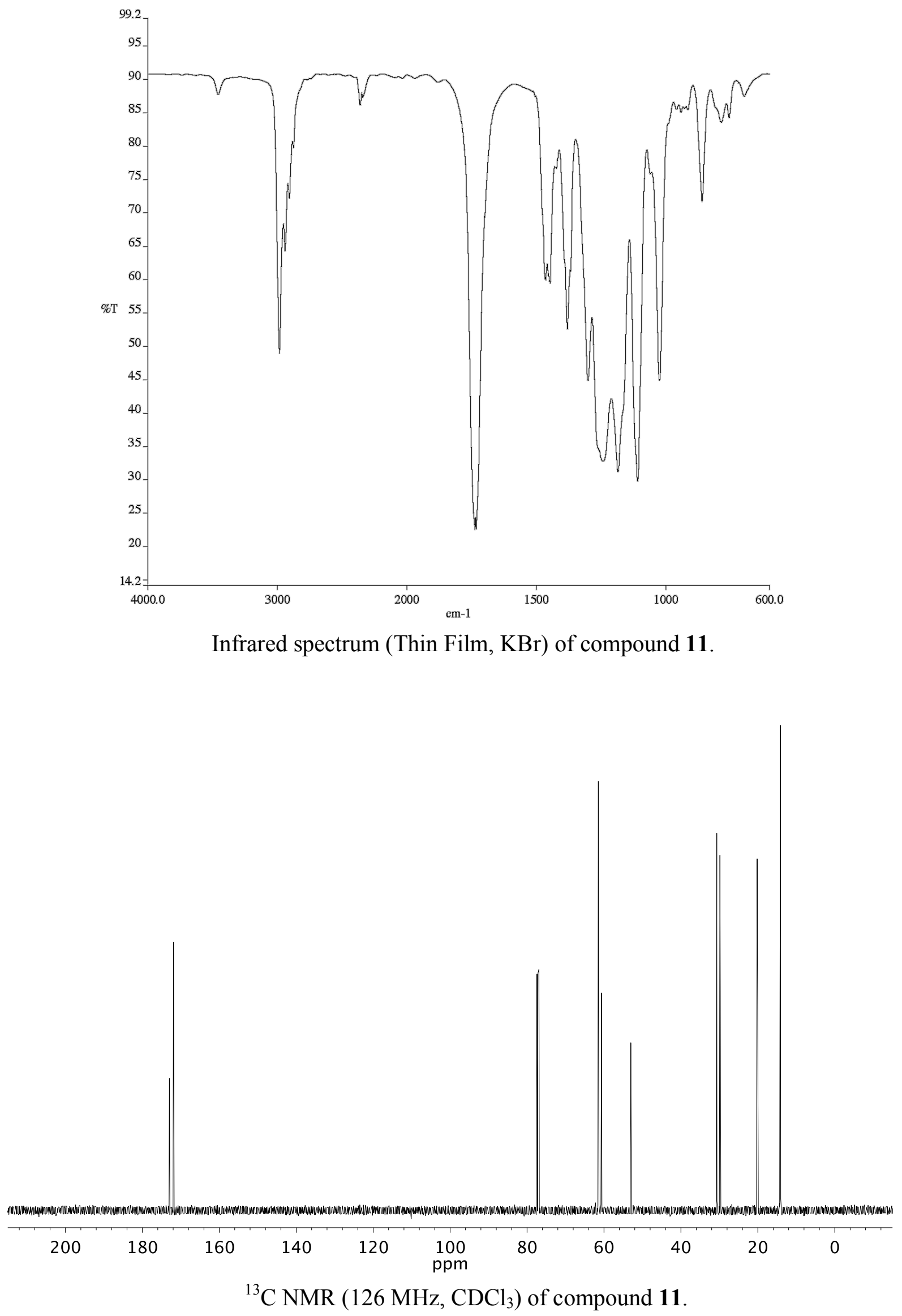


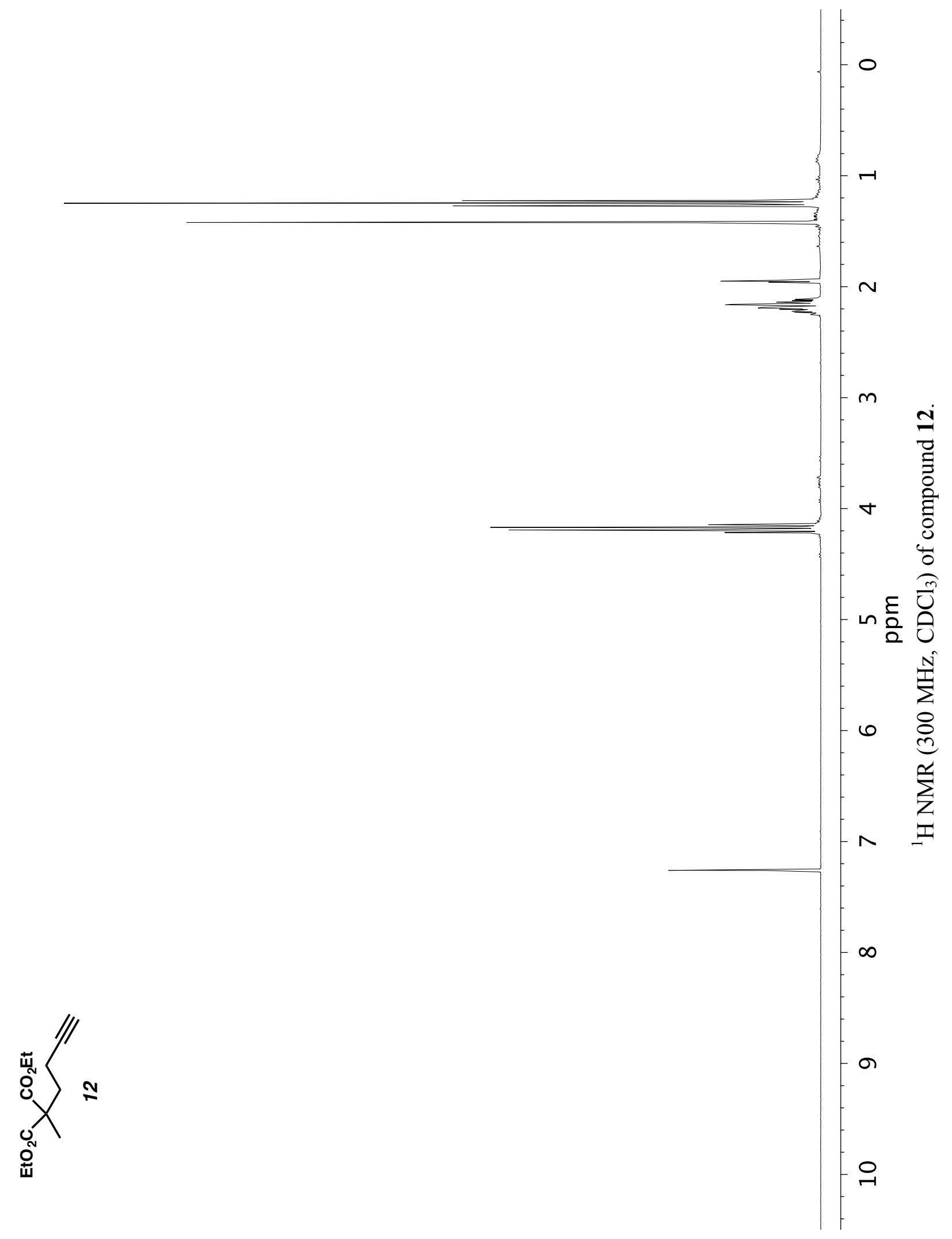



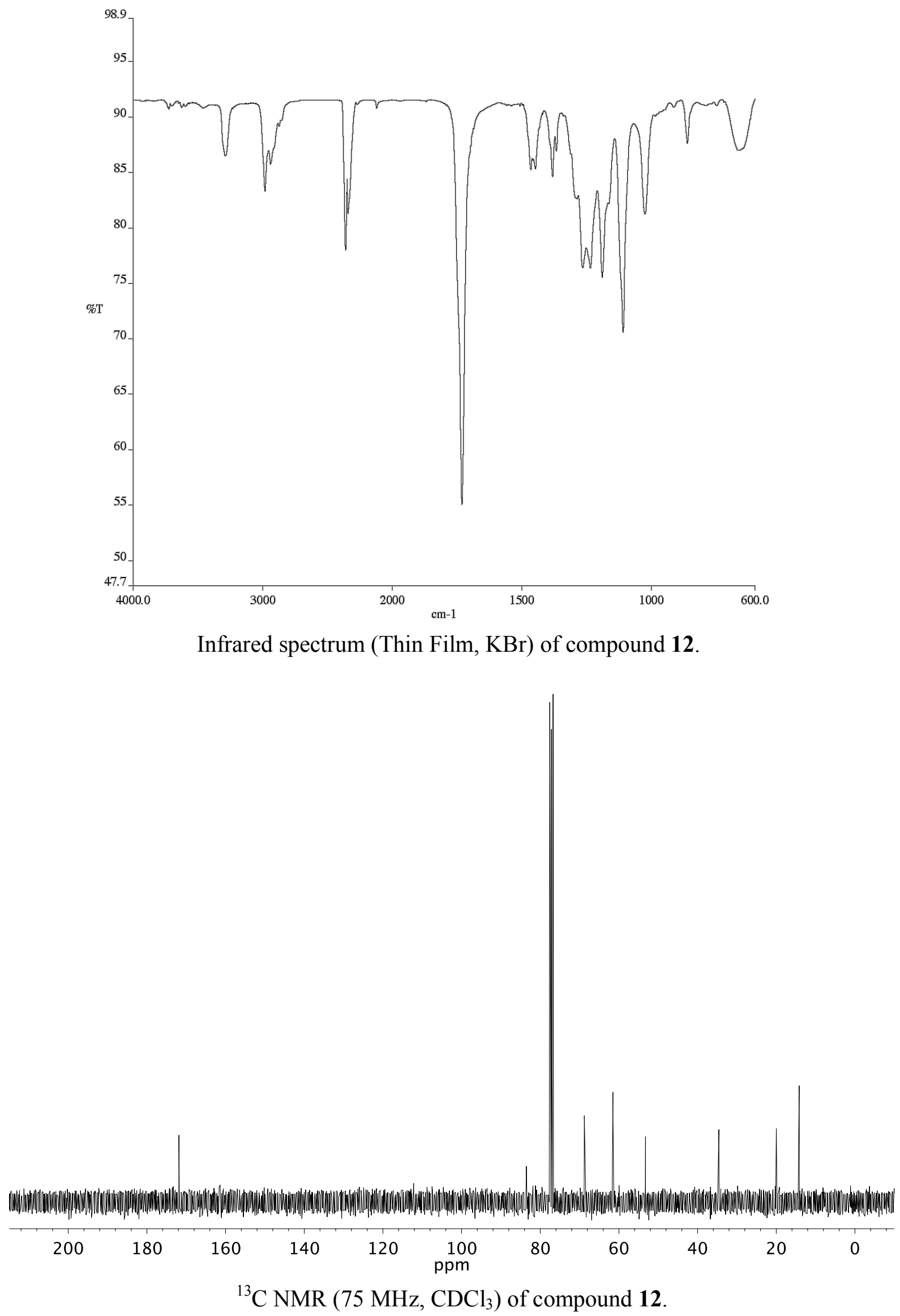